\title{
Generation of initial kinetic distributions for simulation of long-pulse charged particle beams with high space-charge intensity
}

\author{
Steven M. Lund* \\ Lawrence Livermore National Laboratory, Livermore, California 94550, USA \\ Takashi Kikuchi ${ }^{\dagger}$ \\ Department of Electrical and Electronic Engineering, Utsunomiya University, Yohtoh 7-1-2, Utsunomiya 321-8585, Japan \\ Ronald C. Davidson \\ Princeton Plasma Physics Laboratory, Princeton, New Jersey 08543, USA \\ (Received 12 April 2007; revised manuscript received 21 July 2009; published 19 November 2009)
}

\begin{abstract}
Self-consistent Vlasov-Poisson simulations of beams with high space-charge intensity often require specification of initial phase-space distributions that reflect properties of a beam that is well adapted to the transport channel - both in terms of low-order rms (envelope) properties as well as the higher-order phasespace structure. Here, we first review broad classes of kinetic distributions commonly in use as initial Vlasov distributions in simulations of unbunched or weakly bunched beams with intense space-charge fields including the following: the Kapchinskij-Vladimirskij (KV) equilibrium, continuous-focusing equilibria with specific detailed examples, and various nonequilibrium distributions, such as the semiGaussian distribution and distributions formed from specified functions of linear-field Courant-Snyder invariants. Important practical details necessary to specify these distributions in terms of standard accelerator inputs are presented in a unified format. Building on this presentation, a new class of approximate initial kinetic distributions are constructed using transformations that preserve linear focusing, single-particle Courant-Snyder invariants to map initial continuous-focusing equilibrium distributions to a form more appropriate for noncontinuous focusing channels. Self-consistent particle-in-cell simulations are employed to show that the approximate initial distributions generated in this manner are better adapted to the focusing channels for beams with high space-charge intensity. This improved capability enables simulations that more precisely probe intrinsic stability properties and machine performance.
\end{abstract}

DOI: $10.1103 /$ PhysRevSTAB.12.114801

PACS numbers: 29.27.Bd, 41.75.-i, 52.65.Rr, 52.59.Sa

\section{INTRODUCTION}

Numerical simulations of charged particle beams using the Vlasov-Maxwell equations have become an indispensable tool to analyze long-pulse accelerator systems with high space-charge intensity [1-11]. Analytical theory can become very cumbersome for realistic investigations of systems with strong space-charge forces. Meanwhile, the increasing power of digital computers and ever improving numerical methods enable high-level modeling with lesser degrees of idealization. Large-scale computer simulations of Vlasov evolutions using particle-in-cell (PIC) methods adapted from plasma physics $[12,13]$ are routinely used to identify physical mechanisms limiting transport [14-16], to validate practical design concepts [17], and to support interpretation of experiments where only limited diagnostics are possible $[9,18,19]$. In the future, direct Vlasov methods promise improved, low-noise simulations for im-

\footnotetext{
*smlund@1lnl.gov

${ }^{\dagger}$ Present address: Department of Electrical Engineering, Nagaoka University of Technology, Nagaoka 940-2188, Japan.
}

proved understanding of halo particle production and other effects difficult to resolve with PIC methods [20-23]. It is critical in many applications of Vlasov simulations of intense beams to employ initial ("load") distribution functions that are well adapted to the transport lattice. If the total beam propagation distance is not too long and the injector is amenable to modeling, then the beam emission from the source can be simulated and the subsequent evolution through the transport and acceleration cycle of the machine simulated for high-level "first-principles" modeling with limited assumptions [24-27]. Alternatively, the loaded distribution can be synthesized based on reasonable extrapolations of limited experimental measurements of the beam phase space at a diagnostic location in the machine lattice $[28,29]$. Or finally, the initial beam distribution can be assumed to be of a particular (ansatz) form motivated by physical insight or generated by relaxation processes from a relatively simple initial ansatz distribution. The ansatz approach is especially useful when analyzing intrinsic transport limits of beams with high space-charge intensity-particularly if a smooth "equilibrium" beam distribution can be constructed which main- 
tains the functional form of the initial distribution. Then well-established methods of plasma physics [30] can be applied to understand the consequence of system perturbations in a simplified manner.

In continuous-focusing channels the transverse appliedfocusing force is constant as the beam propagates in the lattice. System energy is then conserved and an infinite variety of stationary, stable equilibrium distributions with appealing physical properties can be constructed from distributions that are specified smooth functions of the single-particle Hamiltonian [30-32]. At high space-charge intensity, the self-consistent space-charge forces of the continuous-focusing distributions lead to characteristic Debye-screened density projections that one would expect on physical grounds-with a flat core and a sharp edge [30-32]. Unfortunately, the continuous-focusing model is not in general directly applicable to laboratory systems. Real applied-focusing lattices are typically periodic or quasiperiodic structures where the applied forces vary rapidly with the axial coordinate $s$. This variation of the applied-focusing force vastly complicates the construction of equilibrium or approximate equilibrium distributions and also complicates beam stability [16,33-35]. The $s$-varying applied-focusing fields can transfer energy into and out of the beam, rendering the continuous-focusing model only useful as an approximate, qualitative guide for lattices with relatively weak applied focusing (i.e., low undepressed particle phase advances).

In the presence of finite beam space charge, a wellknown self-consistent transverse equilibrium distribution for a linear applied-focusing channel with arbitrary $s$ variations in the focusing forces is the KapchinskijVladimirskij (KV) distribution [30-32,36]. Although the low-order properties of the KV distribution are appealing physically, the full four-dimensional structure corresponds to a singular, hyperellipsoidal shell in phase space. For strong space charge, this singular structure drives unphysical, higher-order instabilities [31,32,37-44] which limit practical use of the KV distribution for initializing simulations. The KV distribution is the only exact Vlasov equilibrium known that is a function of linear-field Courant-Snyder invariants [45]. Danilov et al. [46] investigate alternative classes of exact kinetic equilibrium distributions for linear forces. These distributions are highly singular, and based on elementary plasma physics considerations, can be expected to be unstable (similar to the KV distribution) in regimes of high space-charge intensity.

Because of the limitations of the singular equilibrium distributions (KV or otherwise) and the lack of known smooth equilibria for focusing channels with $s$-varying applied-focusing forces, approaches to generate initial kinetic distributions for simulations have been taken by assuming specific nonequilibrium ansatz forms or by applying relaxation techniques. Such ansatz-type distributions in common use include the semi-Gaussian distribution which retains the uniform charge-density of the KV model within an elliptical envelope but modifies the local temperature to be Gaussian-distributed and spatially uniform [47]. This results in a beam edge that is not locally in force balance and a spectrum of waves are launched $[16,48,49]$. Depending on the application, such waves may or may not pose a problem. Another ansatz approach employed is to initialize beams that are functions of Courant-Snyder invariants of single-particle orbits in the applied-focusing fields [50,51]. Such distributions are equilibria when space-charge forces are negligible but can launch significant collective waves due to the lack of consistent local force balance in the core of the beam when space-charge forces are a significant fraction of the average applied-focusing forces. Finally, several perturbative theories based on Hamiltonian averaging techniques [32,5258] and canonical transformations [59] have been developed to construct approximate, non-KV beam equilibria in $s$-varying focusing channels. It is expected that the Hamiltonian averaging techniques produce increasingly reliable equilibrium representations at sufficiently weak applied-focusing fields. The Hamiltonian averaging methods are beginning to be implemented and tested in selfconsistent Vlasov simulations [60]. Vlasov simulations of the canonical transformation procedure of Ref. [59] have been carried out and appear to verify near-equilibrium structure for solenoidal periodic-focusing channels but not for strong (quadrupole) periodic focusing.

Relaxation procedures have been developed to partially circumvent the lack of a known smooth equilibrium distribution with $x$-varying focusing forces. The intent behind relaxation methods is to allow phase mixing, nonlinear effects, and collective relaxation processes to effectively relax the core of a nonequilibrium ansatz "initial" distribution to a form better adapted to the applied-focusing channel. These approaches presuppose the existence of a stable underlying equilibrium, which particularly for strong focusing strength may not be the case [16,33-35], thereby limiting applicability. One approach tried is to gradually increase the space-charge intensity by adjusting species weights while evolving the beam [61,62], or similarly by slowly removing nonlinear applied-field components applied to better match the edge, while evolving the beam [63]. Another approach has been to employ "Langevin" procedures where stochastic, scattering terms and damping terms are added to the particle equations of motion and the simulations are advanced until these terms balance while driving the beam to a relaxed state [64]. In these methods it can be difficult to parametrically determine sufficient propagation distances and criteria to specify adequate relaxation [15] and/or how rapidly the spacecharge intensity can be increased toward desired values. Moreover, the desired beam parameters (emittances, etc.) can be difficult to obtain. Recent work by Dorf et al. $[65,66]$ improves the relaxation approach by launching 
continuous-focusing distributions (equilibrium and otherwise) and adiabatically modifying the focusing to periodic form. Encouraging results are obtained by allowing the beam to adapt to the channel while adiabatically reducing the continuous-focusing fields simultaneously with increasing periodic-focusing fields in a manner where nearly constant net focusing strength is maintained.

In this study, conventional initial distributions employed in beam simulations are first reviewed within a common framework. Special attention is applied to generating smooth, stationary equilibria in continuous-focusing channels. Continuous equilibria with "waterbag," "parabolic," and "thermal" forms are analyzed in detail. Procedures are formulated for all types of distributions presented to initialize macroparticles in PIC simulations. Results from these standard distributions are then applied to develop a new class of pseudoequilibrium distributions that are useful for initializing Vlasov simulations of beams in transport channels with $s$-varying applied-focusing forces. The pseudoequilibrium distributions are constructed by transforming continuous-focusing equilibria to rms equivalency [31] with a $\mathrm{KV}$ beam in a manner that preserves the linear space-charge Courant-Snyder invariants. The pseudoequilibrium distributions are not exact equilibria, but are relatively easy to synthesize, and have appealing physical properties that better reflect the relaxed, equilibriumlike form expected in stable transport. The proximity to equilibrium form reduces the initial transient waves associated with the lack of full consistency, thereby simplifying interpretation of the simulations. The procedure is formulated using standard accelerator inputs. Results are illustrated for transverse PIC simulations of fourdimensional (4D) phase space describing an unbunched, coasting beam. The results indicate that the loads will prove superior to standard beam initializations-particularly for high relative space-charge intensity. The method used to generate the pseudoequilibrium distributions also applies to 6D phase-space distributions if the axial particle phase space is specified. Parametric simulation studies carried out with initial pseudoequilibrium distributions have already been applied to better understand the intrinsic space-charge limits in the transport of matched beams in periodic-focusing channels [16,33-35]. Parts of formulations presented here have been developed through extensions of cited material in a series of graduate level classes on beam physics with high space-charge intensity taught by Barnard and Lund at the U.S. Particle Accelerator School [67] and the University of California at Berkeley [68], and Lund at the French National Institute for Research in Computer Science and Controls (INRIA) school on modeling and computational methods for kinetic equations [69].

The organization of this paper is the following. Section II presents an overview of PIC simulation codes employed in this study for testing loaded distributions
(Secs. II A and IIB), and reviews system and numerical parameters employed to characterize simulations (Sec. II C). Classes of transverse distributions commonly employed in simulations are summarized in Sec. III including: equilibrium KV (Sec. III A) and continuousfocusing distributions (Sec. III B), and nonequilibrium semi-Gaussian (Sec. IIIC), and linear-field CourantSnyder invariant (Sec. III D) distributions. Important details are given in the Appendices to allow reasonably abbreviated presentation in the main text without sacrificing completeness. In Appendix A, acceleration effects are analyzed in a manner to allow application of coastingbeam results central to the paper to systems with slow axial acceleration. Appendix B elucidates the important connection between statistical rms emittances and singleparticle Courant-Snyder invariants for particles within a $\mathrm{KV}$ distribution. Appendix $\mathrm{C}$ calculates the moments and projections of distributions of linear-field CourantSnyder invariant distributions presented in Sec. IIID. Appendices D, E, and $\mathrm{F}$ provide extensive details on the equilibrium structure of continuous-focusing waterbag, parabolic, and thermal equilibrium distributions. This includes nonlinear equilibrium properties, solution of constraints to express the solutions in terms of standard accelerator parameters, and simplifications to load the distributions in PIC codes. These results are applied in Sec. IV to construct classes of pseudoequilibrium distributions which improve on several aspects of the conventional distributions reviewed. Straightforward procedures are formulated to construct the distributions (Sec. IVA), and the simulations presented (Sec. IV B) highlight key results. Concluding comments in Sec. V outline the range of usefulness and limitations of the pseudoequilibrium distributions.

\section{SIMULATION DESCRIPTIONS}

Here we briefly describe two electrostatic PIC simulation codes employed and parameter choices made associated with example simulations carried out. This allows succinct presentation of later examples while providing complete details on numerical methods and parameters employed so results can be reproduced. Methods described are mostly standard and can be readily applied within a variety of PIC codes used to simulate charged particle beams with high space-charge intensity. The two codes employed to evaluate the performance of initial distributions analyzed in this study are WARP (Sec. II A) and B-DYN (Sec. II B). Example simulations are carried out on both serial and parallel computer systems using both codes. Code descriptions focus on specific numerical methods employed. For simplicity, transverse simulations are carried out of a coasting beam with a common set of system parameters (Sec. II C). Parameter discussions are framed in a general manner to highlight resolution and statistics issues associated with PIC simulations. 


\section{A. The WARP code}

The WARP code is a versatile set of simulation tools originally developed to model ion beams with intense space charge for application to heavy ion fusion $[1,2,5,70,71]$. Particle moving, fieldsolve, loading, and diagnostic routines are linked to a Python interpreter to enable a wide variety of simulations without modifying the source code. The WARP code has both fully 3D, $r-z$, and transverse 2D (xy) slice PIC packages in addition to a moment package for centroid and envelope descriptions. All PIC packages are multispecies. The transverse slice package is applied for this study which leapfrog advances macroparticles in time with time-advance steps iterated to map macroparticles from slice to slice. Residence corrections are applied when macroparticles enter and exit hardedge quadrupole focusing elements. The kinematics are fully relativistic and leading-order self-magnetic field effects are included by gamma-factor corrections [32,72,73]. Various fieldsolvers can be applied to solve the discretized Poisson equation with detailed conducting structures. Here we employ a fast Fourier transform solver coupled with a capacity matrix solver to implement boundary conditions for a cylindrical, conducting beam pipe on a uniform, square grid. Symmetry conditions can be exploited for more efficient simulations $(a \sim 4 \times$ reduction in simulation time with equivalent statistics and resolution for ideal quadrupoles). A wide variety of initial distributions described in this paper can be loaded as well as "firstprinciples" simulations of ions emitted from space-charge limited flow injectors, and various synthesized distributions based on extrapolations of a limited set of beam properties (usually from measured experimental data). Various ordered and pseudorandom number sequences can be applied when generating particle loads. Diagnostics include particle phase-space projections with transforms to remove linear coherent flows (allowing better visualization of small, nonlinear distribution distortions), and various moment and binned quantities calculated from the distribution (standard moments as well as fluid flows, kinetic temperatures, etc.).

\section{B. The B-DYN code}

The B-DYN code was developed to study high spacecharge intensity beam dynamics during the final beam bunching section in heavy ion fusion drivers [74-76]. The B-DYN code employs a 2D, transverse slice model. When applied to longitudinally compressing beams, species weights are adjusted to model increased transverse space-charge forces resulting from the compression [77]. Macroparticles are advanced in the axial coordinate $s$ using the paraxial approximation, relativistic equations of motion, and the leapfrog method. Axial advance steps are chosen so that macroparticles are not advanced through element boundaries when entering and exiting hard-edge quadrupoles. Leading-order self-magnetic field effects are included using gamma-factor corrections [32,73]. The selffield is calculated on a uniform, square grid by solving the discretized Poisson equation using a multigrid, successive over-relaxation method [78]. Conducting-pipe boundary conditions are taken on the square grid boundary. System symmetries are not exploited. A wide variety of distributions can be loaded. Sequences of pseudorandom numbers are employed in generating particle loads.

\section{Simulation parameters}

In simulations carried out to illustrate distribution loads, we assume a periodic focusing-off-defocusing-off (FODO) quadrupole magnetic-focusing lattice or a continuousfocusing lattice. The periodic FODO lattice has piecewise constant lattice focusing functions $\kappa_{j}$ in the $j=x, y$ planes as illustrated in Fig. 1 with $\kappa_{x}=-\kappa_{y}$. Quadrupole focusing elements have fractional magnet occupancy $\eta$ in the lattice with period $L_{p}=0.5 \mathrm{~m}$. Equal axial-length and equal-strength focusing and defocusing quadrupoles $(\ell=$ $\left.\eta L_{p} / 2\right)$ are separated by equal axial-length drifts $[d=$ $\left.(1-\eta) L_{p} / 2\right]$. The scale of the $\kappa_{j}$ are set by the undepressed particle phase advance $\sigma_{0}$ (measured in degrees) using a formula presented in Ref. [79] $\left(\sigma_{0 x}=\sigma_{0 y} \equiv \sigma_{0}\right)$ for $\sigma_{0}=45^{\circ}$ (for relatively weak focusing), and $\sigma_{0}=70^{\circ}$ (for relatively strong focusing near the stability limit of the lattice [16,33-35]). A pure $K^{+}$ion beam is assumed with $\mathcal{E}_{b}=1 \mathrm{MeV}$ axial particle kinetic energy (nonrelativistic). No spread in axial velocity is taken for simplicity, and the beam is unbunched and coasting (not accelerating) in the periodic lattice. The rms-edge emittance of the beam is set as $\varepsilon_{x}=\varepsilon_{y}=50 \mathrm{~mm}$ mrad [see Eq. (24)] for both strengths of applied-focusing fields considered. The beam line charge $\lambda=$ const is adjusted to obtain specified values of the dimensionless perveance $Q$ [see Eq. (22)]. The initial beam envelope is taken to be rms matched in the lattice according to the $\mathrm{KV}$ envelope equations [see Eq. (21)], and the beam slice is launched at the axial midpoint of a drift before a focusing-in- $x$ quadrupole. Depressed particle phase advances $\sigma\left(\sigma_{x}=\sigma_{y} \equiv \sigma\right)$ are specified for the loaded beam with nonuniform charge

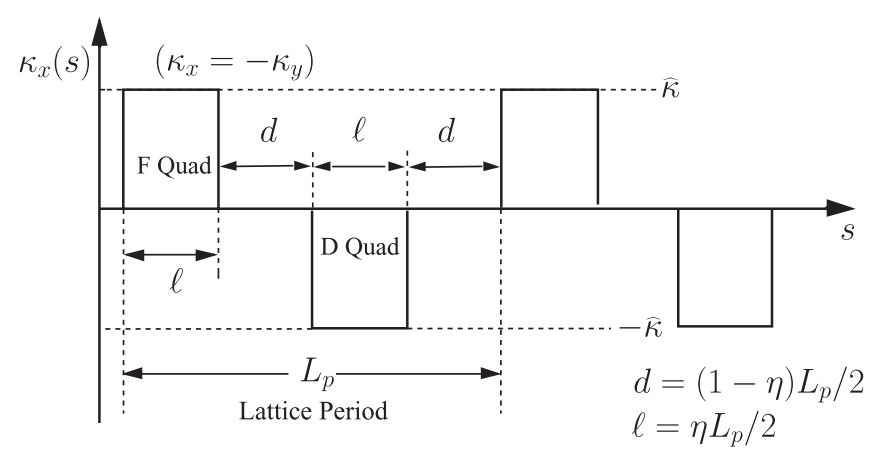

FIG. 1. Quadrupole FODO focusing lattice employed in illustrative simulations. 
density in terms of $\sigma / \sigma_{0}$, calculated from an rmsequivalent matched beam [31] [see Eq. (25)].

For continuous-focusing simulations, the same choices described above for the FODO quadrupole lattice are made, but the applied-focusing functions are set with $\kappa_{j}=$ $k_{\beta 0}^{2}=$ const. To further aid comparisons to FODO lattice simulations, we take (arbitrarily) $k_{\beta 0}=\left(\pi / 180^{\circ}\right) \sigma_{0} / L_{p}$ with $L_{p}=0.5 \mathrm{~m}$.

Numerical parameters of the simulations are set for both high resolution and good statistics (low noise) to better evaluate the subsequent evolution of the distribution loads. Parameter choices are specified for loaded distributions with nonuniform space charge in terms of rms-equivalent beam [31] edge radii $r_{x}$ and $r_{y}$ [see Eq. (23)]. Uniform, rectangular transverse spatial grids are employed with $x$ and $y$-grid increments $\Delta_{x}$ and $\Delta_{y}\left(\Delta_{x}=\Delta_{y}\right)$ chosen for

$$
N_{r}=\sqrt{\frac{r_{x} r_{y}}{\Delta_{x} \Delta_{y}}}
$$

zones (typical $N_{r} \in[20,200]$ ) across the matched-beam radius, with $N_{r}$ sufficiently large to resolve the structure of the beam edge. Round (WARP, with $r_{p}$ radius) or square (B-DYN, with $2 r_{p}$ side length) conducting beam pipes are placed on the grid far enough from the matched envelope excursions with

$$
N_{p}=\frac{r_{p}}{\sqrt{r_{x} r_{y}}},
$$

chosen large enough (typical $N_{p} \simeq 3$ here) to render image-charge effects small. For a given value of $N_{p}$, image-charge effects will be more strongly mitigated in WARP than B-DYN, because the round beam pipe more closely matches the equipotentials external to a roughly elliptical charge symmetry beam than a square pipe. In general, correct image-charge modeling requires implementing boundary conditions associated with the structure of the aperture under consideration in the field solver. The number of macroparticles per grid cell,

$$
N_{\mathrm{ppg}}=\frac{N}{\pi r_{x} r_{y} /\left(\Delta_{x} \Delta_{y}\right)},
$$

where $N$ is the total number of macroparticles loaded, is set large enough (typical $N_{\mathrm{ppg}} \in\left[10^{2}, 10^{4}\right]$ and even larger on parallel machines) to reduce statistical noise on the grid and to produce low noise in binned diagnostic quantities such as densities and kinetic temperatures. Generally, we find that the requirement of reducing noise for clear diagnostics to be more stringent than required for high-fidelity simulations. Symmetry factors are included in measuring $N_{\text {ppg }}$ in WARP simulations. The axial advance stepsize $\Delta_{s}$ of the macroparticles is set for

$$
N_{s}=\frac{L_{p}}{\Delta_{s}}
$$

steps per period (typical $N_{s} \in[100,500]$ ) to resolve both rapidly varying in $s$ applied-focusing forces of the lattice (more restrictive), and evolving collective space-charge waves (generally less restrictive). Total advance lengths in $s$ are carried out over relatively small numbers of lattice periods because the purpose of the present analysis is to evaluate initial transient deviations from the load to stress nonequilibrium like characteristics rather than collective relaxations over longer evolutions [15,48,49]. Diagnostic plots of binned density are contrasted at successive lattice periods to emphasize changes. Spatial binning grids can be independently set from the fieldsolve grid to allow use of coarser diagnostic meshes that reduce noise while resolving relevant features.

The simulation parameters $N_{r}$ and $N_{s}$ should also set consistently with resolving the characteristic Debye screening length $\lambda_{D}=\sqrt{\epsilon_{0} \bar{T} /\left(q^{2} \bar{n}\right)}$ [see Secs. III and Appendix F]. Here, $\bar{T}$ and $\bar{n}$ are characteristic spatially averaged kinetic temperature and density measures over the beam. One expects that the radial falloff distance of the beam density will be related to the Debye length, and that resolving the edge (i.e., $N_{r}$ sufficiently large) will result in the number of cells per Debye length,

$$
N_{D}=\frac{\lambda_{D}}{\sqrt{\Delta_{x} \Delta_{y}}},
$$

being sufficiently large to resolve screening of interactions for high space-charge intensity. Likewise, controlling statistical noise (i.e., $N_{\text {ppg }}$ sufficiently large) on a grid chosen to resolve the Debye length will generally assure that the number of macroparticles within a characteristic Debye screening circle

$$
N_{\mathrm{pp} D}=N \frac{\lambda_{D}^{2}}{r_{x} r_{y}}
$$

is sufficiently large. For charged particle beams with nonuniform temperature and density as well as an effective edge radii $r_{x}$ and $r_{y}$ that evolve in the focusing lattice, issues of adequate resolution of plasma parameters can depend on the specific distribution and application. Although some guidance exists in simple neutral plasma systems $[12,13]$, generally for intense beams these issues must be explored carefully to establish confidence that quantities examined are adequately represented and numerically converged.

\section{FORMULATION AND REVIEW OF INITIAL TRANSVERSE KINETIC DISTRIBUTIONS COMMONLY EMPLOYED IN SIMULATIONS OF LINEAR-FOCUSING CHANNELS}

We consider a beam of particles of charge $q$ and rest mass $m$. The beam can be fully specified by the $\mathbf{x}-\mathbf{p}$ phasespace coordinates of the particles evolving in time. For 
present purposes, we model an axially thin, transverse slice of beam evolving in the accelerator lattice as a function of the axial coordinate $s$ of the slice in the machine. The slice moves axially with velocity $\beta_{b} c=$ const and relativistic gamma factor $\gamma_{b}=1 / \sqrt{1-\beta_{b}^{2}}=$ const. Here, $c$ is the speed of light in vacuo. The transverse phase space of the beam is described by the spatial coordinate $\mathbf{x}_{\perp}=x \hat{\mathbf{x}}+y \hat{\mathbf{y}}$ and the angle $\mathbf{x}_{\perp}^{\prime}$ that the particle makes with the axis of the machine. Primes henceforth denote derivatives with respect to $s$, and in the paraxial approximation, $\mathbf{x}_{\perp}^{\prime} \simeq$ $v_{\perp} /\left(\beta_{b} c\right)$, where $v_{\perp}$ is the transverse particle velocity. In the Vlasov description, the slice is modeled by a continuous, single-particle distribution function $f_{\perp}\left(\mathbf{x}_{\perp}, \mathbf{x}_{\perp}^{\prime}, s\right)$. In the paraxial limit, $f_{\perp}$ evolves as an incompressible fluid in $4 \mathrm{D}$ transverse phase space according to the nonlinear Vlasov equation [30-32,67-69]

$$
\left\{\frac{\partial}{\partial s}+\frac{\partial H_{\perp}}{\partial \mathbf{x}_{\perp}^{\prime}} \cdot \frac{\partial}{\partial \mathbf{x}_{\perp}}-\frac{\partial H_{\perp}}{\partial \mathbf{x}_{\perp}} \cdot \frac{\partial}{\partial \mathbf{x}_{\perp}^{\prime}}\right\} f_{\perp}\left(\mathbf{x}_{\perp}, \mathbf{x}_{\perp}^{\prime}, s\right)=0 .
$$

Here,

$$
H_{\perp}\left(\mathbf{x}_{\perp}, \mathbf{x}_{\perp}^{\prime}\right)=\frac{1}{2} \mathbf{x}_{\perp}^{\prime 2}+\frac{1}{2} \kappa_{x} x^{2}+\frac{1}{2} \kappa_{y} y^{2}+\frac{q}{m \gamma_{b}^{3} \beta_{b}^{2} c^{2}} \phi
$$

is the single-particle Hamiltonian, $\kappa_{j}(s)(j=x, y)$ are the usual functions describing linear applied-focusing forces of the lattice [31,79], and $\phi\left(\mathbf{x}_{\perp}, s\right)$ is the self-field potential generated by the beam space charge. The potential $\phi$ satisfies the transverse Poisson equation,

$$
\nabla_{\perp}^{2} \phi=-\frac{q}{\epsilon_{0}} \int d^{2} x_{\perp}^{\prime} f_{\perp},
$$

with $\phi$ subject to the appropriate boundary conditions on the transverse machine aperture. Here, $\epsilon_{0}$ is the permittivity of free space.

The Vlasov-Poisson system given by Eqs. (8) and (9) models the transverse beam evolution in the continuum approximation. The system is solved as an initial value problem where $f_{\perp}\left(\mathbf{x}_{\perp}, \mathbf{x}_{\perp}^{\prime}, s\right)$ is specified at some initial value of $s=s_{i}$. Any positive-definite distribution function formed from a set of single-particle constants of the motion $\left\{C_{i}\right\}$ will produce a valid, exact "equilibrium" solution to the Vlasov equation. Here, the notion of equilibrium is that the form of $f_{\perp}=f_{\perp}\left(\left\{C_{i}\right\}\right)$ does not evolve in $s$. A special case of this is a stationary equilibrium with $\partial f_{\perp} / \partial s=0$. Stationary beam equilibria occur in continuous-focusing systems with $\kappa_{j}=$ const and are extensively analyzed in Sec. III B. Self-consistency requires that the equilibrium distribution $f_{\perp}\left(\left\{C_{i}\right\}\right)$ generates the required self-field configuration needed for validity of the constants of the motion. Exact self-consistency is highly nontrivial for focusing channels with $s$-varying applied-focusing forces described by $\kappa_{j}(s)$.
In direct Vlasov simulations, a specified initial $\left(s=s_{i}\right)$ distribution $f_{\perp}\left(\mathbf{x}_{\perp}, \mathbf{x}_{\perp}^{\prime}, s=s_{i}\right)$ need only be loaded on the phase-space grid of the simulation. For distributions without singularities or sharp edges the distribution loading for direct Vlasov codes is straightforward. The spatial $\mathbf{x}_{\perp}$ and angle $\mathbf{x}_{\perp}^{\prime}$ grid of the simulation should, of course, be chosen accordingly to resolve distribution variations in phase space. For beams with sharp edges or discontinuities, there will generally be errors involved in discretizing the distribution unless numerical methods that are specific to the type of distribution are employed. For more customary PIC simulations [12], a finite distribution of macroparticles must be synthesized to represent the continuous initial Vlasov distribution. Although the PIC method can simplify the treatment of distributions with sharp edges or discontinuities, sufficiently large numbers of macroparticles must be employed to adequately sample the distribution and limit statistical noise associated with the discretized representation. Undesired correlations between macroparticles must be prevented. Generally, procedures are formulated to load phase-space coordinates exploiting distribution symmetries and using probability transforms of pseudorandom uniform deviates typically available in mathematical library functions. This is generally preferable to Monte Carlo sampling of $f_{\perp}$ due to statistical noise issues. Examples of explicit macroparticle initialization methods for various distributions will be discussed in subsequent sections.

Equations relating the focusing functions $\kappa_{j}$ to magnetic and/or electric fields of practical focusing elements are presented in Refs. [31,79]. If the lattice has nonlinear applied fields, appropriate terms can be added the Vlasov equation (7) and the $\kappa_{j}$ functions describe only the linear component focusing terms (excluding skew couplings). The $\kappa_{j}$ can be periodic in $s$ or not. For periodic lattices, the scale of the $\kappa_{j}$ can be regarded as fixed by the undepressed phase advances $\sigma_{0 j}$ (measured in degrees per lattice period) of a single particle evolving in the absence of the beam in the linear applied fields of the lattice $[31,79]$. For the FODO quadrupole lattice used in illustrative simulations in this paper, $\sigma_{0 x}=\sigma_{0 y} \equiv \sigma_{0}$.

The beam line-charge density,

$$
\lambda \equiv q \int d^{2} x_{\perp} \int d^{2} x_{\perp}^{\prime} f_{\perp},
$$

is constant $(\lambda=$ const $)$ in slice models when particles are not lost from the system. In later 3D generalizations $\lambda$ will be allowed to vary with $s$ in a specified manner. Statistical averages over the full transverse phase space of the beam slice are denoted by

$$
\langle\cdots\rangle_{\perp} \equiv \frac{\int d^{2} x_{\perp} \int d^{2} x_{\perp}^{\prime} \cdots f_{\perp}}{\int d^{2} x_{\perp} \int d^{2} x_{\perp}^{\prime} f_{\perp}},
$$

and restricted angle averages over $\mathbf{x}_{\perp}$ by 


$$
\langle\cdots\rangle_{\mathbf{x}_{\perp}^{\prime}} \equiv \frac{\int d^{2} x_{\perp}^{\prime} \cdots f_{\perp}}{\int d^{2} x_{\perp}^{\prime} f_{\perp}} .
$$

We will frequently employ distribution moments such as the number density of beam particles

$$
n \equiv \int d^{2} x_{\perp}^{\prime} f_{\perp},
$$

the $x$ - and $y$-plane coherent flow angles $\left\langle x^{\prime}\right\rangle_{\mathbf{x}_{\perp}^{\prime}}$ and $\left\langle y^{\prime}\right\rangle_{\mathbf{x}_{\perp}^{\prime}}$, and the incoherent flows (i.e., effective kinetic temperatures)

$$
T_{x} \equiv\left\langle\left(x^{\prime}-\left\langle x^{\prime}\right\rangle_{\mathbf{x}_{\perp}^{\prime}}\right)^{2}\right\rangle_{\mathbf{x}_{\perp}^{\prime}}=\left\langle x^{\prime 2}\right\rangle_{\mathbf{x}_{\perp}^{\prime}}-\left\langle x^{\prime}\right\rangle_{\mathbf{x}_{\perp}^{\prime}}^{2},
$$

with an analogous equation for $T_{y}$. Moments that are formed by integrating over degrees of freedom of the distribution $f_{\perp}$ are sometimes called projections (e.g., the density $n$ is the $x-y$ distribution projection). Generally, to account for centroid motion, transverse phase-space coordinates are measured relative to the charge center of mass of the beam using

$$
\tilde{\mathbf{x}}_{\perp}=\mathbf{x}_{\perp}-\left\langle\mathbf{x}_{\perp}\right\rangle_{\perp}, \quad \tilde{\mathbf{x}}_{\perp}^{\prime}=\mathbf{x}_{\perp}^{\prime}-\left\langle\mathbf{x}_{\perp}^{\prime}\right\rangle_{\perp} .
$$

For notational simplicity, we henceforth assume an on-axis centroid with $\left\langle\mathbf{x}_{\perp}\right\rangle_{\perp}=0=\left\langle\mathbf{x}_{\perp}^{\prime}\right\rangle_{\perp}$. It is straightforward to modify results presented for nonzero centroid evolution by replacing $\mathbf{x}_{\perp} \rightarrow \tilde{\mathbf{x}}_{\perp}$.

If the beam slice is accelerated axially by specified longitudinal forces, then $\gamma_{b} \beta_{b} \neq$ const is allowed to vary as some prescribed function of $s$. It is shown in Appendix A that, if the acceleration is slowly varying, then the formulation presented above is applicable when interpreted in terms of appropriately transformed variables. Consequently, results presented for $\gamma_{b} \beta_{b}=$ const here and in subsequent sections can also be applied to accelerating beams provided that variables are consistently interpreted. This remains true even if the beam is axially long and $\gamma_{b} \beta_{b}$ varies from the head to the tail of the pulse.

\section{A. The Kapchinskij-Vladimirskij equilibrium distribution}

The so-called KV equilibrium distribution was constructed by Kapchinskij-Vladimirskij [36] and has been extensively studied [30-32,37-45,67-69]. Here, we review properties of the distribution for later use in formulating alternative, smooth distributions to load. The KV distribution can be symmetrically expressed as

$$
\begin{aligned}
f_{\perp}\left(\mathbf{x}_{\perp}, \mathbf{x}_{\perp}^{\prime}, s\right)= & \frac{\lambda}{q \pi^{2} \varepsilon_{x} \varepsilon_{y}} \delta\left[\left(\frac{x}{r_{x}}\right)^{2}+\left(\frac{r_{x} x^{\prime}-r_{x}^{\prime} x}{\varepsilon_{x}}\right)^{2}\right. \\
& \left.+\left(\frac{y}{r_{y}}\right)^{2}+\left(\frac{r_{y} y^{\prime}-r_{y}^{\prime} y}{\varepsilon_{y}}\right)^{2}-1\right] .
\end{aligned}
$$

Here, $\delta(x)$ is the Dirac delta function $[\delta(x)=0$ for $x \neq 0$ and $\int d x f(x) \delta(x)=f(0)$ for any integrable function $f(x)$ ], $r_{j}=r_{j}(s)(j=x, y)$ are the edge (envelope) radii of the uniform-density elliptical beam core, $r_{j}^{\prime}=r_{j}^{\prime}(s)$ are the envelope angles, and $\varepsilon_{j}=$ const are the rms-edge emittances of the beam. The KV distribution is an exact equilibrium solution of the Vlasov equation (7) in the absence of nonlinear image-charge forces (axisymmetric system with $\partial / \partial \theta=0$, or free-space approximation) $[30,32,36]$. This follows because the KV distribution is a function of singleparticle Courant-Snyder invariants of the linear applied focusing and linear space-charge defocusing forces generated by the distribution itself (see Appendix B), and therefore an initial distribution with the $\mathrm{KV}$ form given by Eq. (16) maintains the KV form for all $s$. Using techniques similar to those employed in the derivation of the density inversion theorem in continuous-focusing channels (see Sec. III B), it can be shown that the delta-function form of Eq. (16) arises naturally to produce a uniform-density elliptical beam consistent with Courant-Snyder invariant forms $[67,68]$. Canonical transforms can also be applied to equivalently express a wide variety of superficially different appearing expressions of the KV distribution in symmetrical canonical form [i.e., $f(\mathbf{q}, \mathbf{p}) \propto \delta\left(\mathbf{q}^{2}+\mathbf{p}^{2}-1\right)$ for 2D canonical variables $\mathbf{q}$ and $\mathbf{p}$ ] [45]. Danilov et al. [46] investigate alternative classes of exact kinetic equilibrium distributions for linear forces using both Courant-Snyder and Wronskian-type invariants of the particle orbits. These distributions are highly singular in an analogous sense to the KV distribution, and in several cases represent theories that are cold in one or more phase-space planes. The added Wronskian invariant appears to allow construction of linear-force distributions in 3D when one or more phasespace planes are cold.

Projections and moments of the KV distribution are most readily calculated using canonical transforms (see Appendix C and Refs. [30,67,68]). All two-dimensional (2D) phase-space projections of the KV distribution correspond to uniformly filled ellipses. The orientation and shape of the elliptical projections evolve in $s$ as the beam propagates in the lattice. The density $n$ of the KV distribution (i.e., the $x-y$ projection) is uniform within an elliptical beam envelope with

$$
n=\int d^{2} x_{\perp}^{\prime} f_{\perp}= \begin{cases}\frac{\lambda}{q \pi r_{x} r_{y}}, & \text { if }\left(\frac{x}{r_{x}}\right)^{2}+\left(\frac{y}{r_{y}}\right)^{2}<1, \\ 0, & \text { otherwise. }\end{cases}
$$

This uniform-density beam produces linear self-field forces within the beam when nonlinear image-charge effects are absent (free-space or aperture sufficiently large). Two-dimensional $x-x^{\prime}$ and $y-y^{\prime}$ phase-space projections can be calculated as

$$
\begin{aligned}
& \int d y \int d y^{\prime} f_{\perp}= \begin{cases}\frac{\lambda}{q \pi \varepsilon_{x}}, & \text { if }\left(\frac{x}{r_{x}}\right)^{2}+\left(\frac{r_{x} x^{\prime}-r_{x}^{\prime} x}{\varepsilon_{x}}\right)^{2}<1, \\
0, & \text { otherwise, }\end{cases} \\
& \int d x \int d x^{\prime} f_{\perp}= \begin{cases}\frac{\lambda}{q \pi \varepsilon_{y}}, & \text { if }\left(\frac{y}{r_{y}}\right)^{2}+\left(\frac{r_{y} y^{\prime}-r_{y}^{\prime} y}{\varepsilon_{y}}\right)^{2}<1, \\
0, & \text { otherwise. }\end{cases}
\end{aligned}
$$


TABLE I. Moments of the KV distribution. All second-order moments not listed vanish (i.e., $\int d^{2} x_{\perp}^{\prime} x y f_{\perp}=0,\langle x y\rangle_{\perp}=0$ ).

\begin{tabular}{lc}
\hline \hline Moment & Value \\
\hline $\int d^{2} x_{\perp}^{\prime} x^{\prime} f_{\perp}$ & $r_{x}^{\prime} \frac{x}{r_{x}} n$ \\
$\int d^{2} x_{\perp}^{\prime} y^{\prime} f_{\perp}$ & $r_{y}^{\prime} \frac{y}{r_{y}} n$ \\
$\int d^{2} x_{\perp}^{\prime} x^{\prime 2} f_{\perp}$ & {$\left[r_{x}^{\prime 2} \frac{x^{2}}{r_{x}^{2}}+\frac{\varepsilon_{x}^{2}}{2 r_{x}^{2}}\left(1-\frac{x^{2}}{r_{x}^{2}}-\frac{y^{2}}{r_{y}^{2}}\right)\right] n$} \\
$\int d^{2} x_{\perp}^{\prime} y^{\prime 2} f_{\perp}$ & {$\left[r_{y}^{\prime 2} \frac{y^{2}}{r_{y}^{2}}+\frac{\varepsilon_{y}^{2}}{2 r_{y}^{2}}\left(1-\frac{x^{2}}{r_{x}^{2}}-\frac{y^{2}}{r_{y}^{2}}\right)\right] n$} \\
$\int d^{2} x_{\perp}^{\prime} x x^{\prime} f_{\perp}$ & $\frac{r_{x}^{\prime}}{r_{x}} x^{2} n$ \\
$\int d^{2} x_{\perp}^{\prime} y y^{\prime} f_{\perp}$ & $\frac{r_{y}^{\prime}}{r_{y}} y^{2} n$ \\
$\int d^{2} x_{\perp}^{\prime}\left(x y^{\prime}-y x^{\prime}\right) f_{\perp}$ & 0 \\
$\left\langle x^{2}\right\rangle_{\perp}$ & $\frac{r_{x}^{2}}{4}$ \\
$\left\langle y^{2}\right\rangle_{\perp}$ & $\frac{r_{y}^{2}}{4}$ \\
$\left\langle x^{\prime 2}\right\rangle_{\perp}$ & $\frac{r_{x}^{2}}{4}+\frac{\varepsilon_{x}^{2}}{4 r_{x}^{2}}$ \\
$\left\langle y^{\prime 2}\right\rangle_{\perp}$ & $\frac{r_{y}^{\prime 2}}{4}+\frac{\varepsilon_{y}^{2}}{4 r_{y}^{2}}$ \\
$\left\langle x x^{\prime}\right\rangle_{\perp}$ & $\frac{r_{x} r_{x}^{\prime}}{4}$ \\
$\left\langle y y^{\prime}\right\rangle_{\perp}$ & $\frac{r_{y} r_{y}^{\prime}}{4}$ \\
$\left\langle x y^{\prime}-y x^{\prime}\right\rangle_{\perp}$ & 0 \\
$16\left[\left\langle x^{2}\right\rangle_{\perp}\left\langle x^{\prime 2}\right\rangle_{\perp}-\left\langle x x^{\prime}\right\rangle_{\perp}^{2}\right]$ & 0 \\
$16\left[\left\langle y^{2}\right\rangle_{\perp}\left\langle y^{\prime 2}\right\rangle_{\perp}-\left\langle y y^{\prime}\right\rangle_{\perp}^{2}\right]$ & $\varepsilon_{x}^{2}$ \\
\hline \hline & $\varepsilon_{y}^{2}$ \\
\hline \hline
\end{tabular}

First- and second-order moments of the KV distribution are summarized in Table I. From these moments, the $j$-plane coherent flow angles are

$$
\begin{aligned}
& \left\langle x^{\prime}\right\rangle_{\mathbf{x}_{\perp}^{\prime}}= \begin{cases}r_{x}^{\prime} \frac{x}{r_{x}}, & \text { if }\left(\frac{x}{r_{x}}\right)^{2}+\left(\frac{y}{r_{y}}\right)^{2}<1, \\
0, & \text { otherwise, }\end{cases} \\
& \left\langle y^{\prime}\right\rangle_{\mathbf{x}_{\perp}^{\prime}}= \begin{cases}r_{y}^{\prime} \frac{y}{r_{y}}, & \text { if }\left(\frac{x}{r_{x}}\right)^{2}+\left(\frac{y}{r_{y}}\right)^{2}<1, \\
0, & \text { otherwise, }\end{cases}
\end{aligned}
$$

and the kinetic temperatures [i.e., incoherent flows, see Eq. (14)] are

$$
T_{j}= \begin{cases}\frac{\varepsilon_{j}^{2}}{2 r_{j}^{2}}\left(1-\frac{x^{2}}{r_{x}^{2}}-\frac{y^{2}}{r_{y}^{2}}\right), & \text { if }\left(\frac{x}{r_{x}}\right)^{2}+\left(\frac{y}{r_{y}}\right)^{2}<1, \\ 0, & \text { otherwise. }\end{cases}
$$

These parabolic temperature profiles drop to zero at the beam edge, which is consistent with linear thermal pressure and a sharp beam edge.

Although the full four-dimensional KV distribution (16) is a manifest (hypershell) invariant, projections of the beam evolve in $s$. In the absence of perturbations (applied field, perturbed distribution, induced image charges, etc.), the envelope radii $r_{j}$ evolve according to the so-called $\mathrm{KV}$ envelope equations [31,36,79]:

$$
r_{j}^{\prime \prime}+\kappa_{j} r_{j}-\frac{2 Q}{r_{x}+r_{y}}-\frac{\varepsilon_{j}^{2}}{r_{j}^{3}}=0
$$

Here,

$$
Q=\frac{q \lambda}{2 \pi \epsilon_{0} m c^{2} \gamma_{b}^{3} \beta_{b}^{2}}=\text { const }
$$

is the dimensionless perveance. If the focusing functions $\kappa_{j}$ are periodic in $s$, and the initial beam parameters are "matched," then the solution for $r_{j}$ will have the same periodicity as the lattice. This, in general, requires specific choices for the envelope functions $r_{j}$ and angles $r_{j}^{\prime}$ at the axial coordinate $s$ where the distribution is specified $[31,79]$. An efficient procedure for numerically calculating the matched-beam envelope under various parameter specifications is presented in Ref. [80].

For the KV distribution, the envelope radii $r_{j}$, the envelope angles $r_{j}^{\prime}$, and the emittances $\varepsilon_{j}=$ const are related to second-order statistical moments of the distribution (see also Table I) as

$$
\begin{array}{ll}
r_{x}=2\left\langle x^{2}\right\rangle_{\perp}^{1 / 2}, & r_{y}=2\left\langle y^{2}\right\rangle_{\perp}^{1 / 2}, \\
r_{x}^{\prime}=2 \frac{\left\langle x x^{\prime}\right\rangle_{\perp}}{\left\langle x^{2}\right\rangle_{\perp}^{1 / 2}}, & r_{y}^{\prime}=2 \frac{\left\langle y y^{\prime}\right\rangle_{\perp}}{\left\langle y^{2}\right\rangle_{\perp}^{1 / 2}}
\end{array}
$$

and

$$
\begin{aligned}
& \varepsilon_{x}=4\left[\left\langle x^{2}\right\rangle_{\perp}\left\langle x^{\prime 2}\right\rangle_{\perp}-\left\langle x x^{\prime}\right\rangle_{\perp}^{2}\right]^{1 / 2}, \\
& \varepsilon_{y}=4\left[\left\langle x^{2}\right\rangle_{\perp}\left\langle x^{\prime 2}\right\rangle_{\perp}-\left\langle x x^{\prime}\right\rangle_{\perp}^{2}\right]^{1 / 2} .
\end{aligned}
$$

When the envelope $r_{j}$ is matched to a periodic-focusing lattice, the depressed phase advance of particles oscillating within the core of the beam under the action of linear applied-focusing fields and linear space-charge defocusing fields can be calculated from [30,31,79]

$$
\sigma_{j}=\varepsilon_{j} \int_{0}^{L_{p}} \frac{d s}{r_{j}^{2}}
$$

where $L_{p}$ is the lattice periodicity length. The ratio of depressed to undepressed phase advance $\sigma_{j} / \sigma_{0 j}$, also called the tune depression, provides a convenient measure of relative space-charge strength with $\sigma_{j} / \sigma_{0 j} \rightarrow 1$ in the limit of vanishingly small space-charge strength $(Q=0)$, and $\sigma_{j} / \sigma_{0 j} \rightarrow 0$ in the limit of maximum space-charge strength $\left(\varepsilon_{j}=0\right)$. For systems with symmetry between the $x$ and $y$ planes that result in $\sigma_{x}=\sigma_{y}$, we denote $\sigma_{j} \equiv \sigma$ for notational simplicity.

Although Eqs. (23)-(25) apply to a KV beam, they are often used to characterize non-KV distributions in an "rms-equivalent" sense [31,81], where a non-KV distribution (with generally nonlinear beam self-fields internal to the beam) is replaced by a KV distribution with the same energy, line charge, and first- and second-order moments as the actual beam. Sacherer's study in Ref. [81] shows that an unbunched beam with charge density constant on elliptical surfaces (i.e., with $x^{2} / r_{x}^{2}+y^{2} / r_{y}^{2}=$ const) obeys the $\mathrm{KV}$ envelope equation (21) provided the envelope radii $r_{j}$ and emittances $\varepsilon_{j}$ are calculated with the generalized beam 
using the averages defined in Eqs. (23) and (24). However, for non-KV beams the rms-edge emittances $\varepsilon_{j}$ defined by Eq. (24) generally evolve in $s[82,83]$.

The KV envelope equation (21) can be employed with self-consistent $s$-varying emittances [defined by Eq. (24)] as an average force-balance equation for statistical edge radii $r_{j}$ [defined by Eq. (23)] that describe a wide variety of distributions $[31,79,81]$. If the emittance variations have a negligible effect on the evolution of the $r_{j}$, then the $\mathrm{KV}$ equation can be applied with $\varepsilon_{j}=$ const for low-order system modeling. Bands of parametric instability described by the KV envelope equations predict parameter regions where machines cannot reliably operate $[31,79,84]$. Unfortunately, the singular structure of the KV distribution leads to unphysical, higher-order collective mode instabilities [31,32,37-44] that render the distribution generally unsuitable to employ as an initial distribution function in Vlasov simulations of beams with high space-charge intensity. Further reducing the applicability of the KV distribution, Neuman has shown that KV distribution (16) in $4 \mathrm{D}$ phase space is not generalizable to 6D phase space (see Appendix A of Ref. [45]). This follows from bounds established by Neuman which show that the density projection of any distribution of linearforce Courant-Snyder invariants in 6D phase space cannot produce a uniform-density ellipsoidal bunch projection in 3D necessary for linear space-charge forces and selfconsistency. The authors of Ref. [46] investigate classes of $6 \mathrm{D}$ phase-space distributions with linear forces by exploiting Courant-Snyder and Wronskian symmetries simultaneously. However, these distributions are highly singular in a similar manner to the KV case. Elementary plasma physics considerations suggest that they can be expected to be unstable (similar to the KV distribution) in regimes of high space-charge intensity.

Loading the initial KV distribution (16) in a direct Vlasov code can be challenging due to the singular (delta-function) structure of $f_{\perp}$. Optimal loading procedures generally center on how to best represent the singular delta function defining the hypershell surface in $4 \mathrm{D}$ phase space where $f_{\perp}=$ const on the discrete phase-space grid of the simulation. For PIC simulations, an initial KV distribution can be loaded several ways. In one approach, the fact that the Courant-Snyder invariant argument of the delta function define a hyperellipsoidal shell in $4 \mathrm{D} \mathbf{x}_{\perp}-\mathbf{x}_{\perp}^{\prime}$ phase space with

$$
\left(\frac{x}{r_{x}}\right)^{2}+\left(\frac{r_{x} x^{\prime}-r_{x}^{\prime} x}{\varepsilon_{x}}\right)^{2}+\left(\frac{y}{r_{y}}\right)^{2}+\left(\frac{r_{y} y^{\prime}-r_{y}^{\prime} y}{\varepsilon_{y}}\right)^{2}=1
$$

can be employed. A finite number of macroparticles can be loaded with phase-space coordinates uniformly distributed on the $4 \mathrm{D}$ hyperellipsoid (or to the extent possible with a finite number of particles) defined by Eq. (26).
In another macroparticle loading approach, the KV distribution property that $\int d x_{1} \int d x_{2} f_{\perp}$ is a uniformly filled ellipse when $x_{1}$ and $x_{2}$ are chosen to be any two of the phase-space coordinates $x, y, x^{\prime}$, and $y^{\prime}$ can be exploited. This approach has the virtue that techniques developed can be generalized to apply to loading other classes of distributions (see Secs. III B and IV). One procedure [85] based on uniform elliptical projections is to first load macroparticle coordinates $\mathbf{x}_{\perp}$ consistent with uniform beam density within the elliptical envelope radii $r_{j}$ [see Eq. (17)]. This can be accomplished using two independent, uniformly distributed random numbers $\hat{u}_{r} \in[0,1)$ and $\hat{u}_{\theta} \in[0,1)$ for each macroparticle and taking

$$
x=r_{x} \sqrt{\hat{u}_{r}} \cos \left(2 \pi \hat{u}_{\theta}\right), \quad y=r_{y} \sqrt{\hat{u}_{r}} \sin \left(2 \pi \hat{u}_{\theta}\right) .
$$

Equation (27) is readily derived by transforming a uniformly filled unit disk to a uniformly filled ellipse with major radii $r_{j}$. With spatial coordinates set according to Eq. (27), macroparticle angles $\mathbf{x}_{\perp}^{\prime}$ can be resolved into coherent and incoherent components as

$$
\mathbf{x}_{\perp}^{\prime}=\left.\mathbf{x}_{\perp}^{\prime}\right|_{\mathrm{c}}+\left.\mathbf{x}_{\perp}^{\prime}\right|_{\mathrm{ic}},
$$

with the coherent (i.e., generally $\left\langle\left.\mathbf{x}_{\perp}^{\prime}\right|_{c}\right\rangle_{\mathbf{x}_{\perp}^{\prime}} \neq 0$ ) components set consistently with Eq. (19) as

$$
\left.x^{\prime}\right|_{\mathrm{c}}=r_{x}^{\prime} \frac{x}{r_{x}},\left.\quad y^{\prime}\right|_{\mathrm{c}}=r_{y}^{\prime} \frac{y}{r_{y}},
$$

and the incoherent (i.e., $\left\langle\left.\mathbf{x}_{\perp}^{\prime}\right|_{\mathrm{ic}}\right\rangle_{\mathbf{x}_{\perp}^{\prime}}=0$ ) components constrained [see Eq. (26)] to satisfy

$$
\left(\frac{\left.r_{x} x^{\prime}\right|_{\mathrm{ic}}}{\varepsilon_{x}}\right)^{2}+\left(\frac{\left.r_{y} y^{\prime}\right|_{\mathrm{ic}}}{\varepsilon_{y}}\right)^{2}=1-\frac{x^{2}}{r_{x}^{2}}-\frac{y^{2}}{r_{y}^{2}} .
$$

Incoherent angles can be generated consistent with this constraint without introducing correlations by using another independent, uniformly distributed random number $\hat{u}_{\varphi} \in[0,1)$ for each macroparticle and taking

$$
\begin{aligned}
& \left.x^{\prime}\right|_{\text {ic }}=\frac{\varepsilon_{x}}{r_{x}} \sqrt{1-\frac{x^{2}}{r_{x}^{2}}-\frac{y^{2}}{r_{y}^{2}}} \cos \left(2 \pi \hat{u}_{\varphi}\right), \\
& \left.y^{\prime}\right|_{\text {ic }}=\frac{\varepsilon_{y}}{r_{y}} \sqrt{1-\frac{x^{2}}{r_{x}^{2}}-\frac{y^{2}}{r_{y}^{2}}} \cos \left(2 \pi \hat{u}_{\varphi}\right) .
\end{aligned}
$$

The finite number of macroparticles loaded can never exactly represent the distribution. There will always be statistical errors resulting from finite statistics and the shape of the macroparticles employed. Also, the discrete spatial grid employed in the PIC method will generally introduce errors in resolving the sharp edge of the KV distribution. For cases where the KV distribution is unstable, these statistical and gridding errors will generally project on unstable collective modes complicating applications of the KV distribution [31,38,39]. 
Statistical noise associated with loads of a finite number of macroparticles in PIC simulations can be substantially reduced by replacing the pseudorandom numbers $\hat{u}_{r}, \hat{u}_{\theta}$, and $\hat{u}_{\varphi}$ with ordered sets of numbers (based on digitreversed numbers, Fibonacci numbers, etc.) in the interval $[0,1)$ to obtain more uniform macroparticle spacing in phase space [12]. Such techniques are especially useful for high-resolution tests of equilibrium loads. However, as simulations are advanced in $s$, noise will eventually grow to levels consistent with the underlying statistics and discretizations associated with the numerical methods employed. Considerable care should be taken when using ordered numbers to load both macroparticle coordinates $\mathbf{x}_{\perp}$ and angles $\mathbf{x}_{\perp}^{\prime}$ that unphysical phase-space correlations are not introduced via the systematic orderings. The possibility of introducing unwanted correlations can be mitigated (at the expense of more load noise) by using ordered numbers only in the particle coordinate or angle loads, but not both. Comments given here on the application of ordered sets of numbers in generating $\mathrm{KV}$ distribution loads are also applicable to loads developed for other distributions in subsequent sections.

Numerous examples of KV beam Vlasov simulations can be found in the literature $[31,38,39,43,59]$ and will not be repeated here. Intrinsic instabilities of the distribution are generally seeded by noise and errors specific to the loading method and numerical approximations employed. This renders results difficult to interpret, particularly for strong relative space-charge strength.

\section{B. Continuous-focusing equilibrium distributions}

The continuous-focusing model has been extensively studied by Davidson and Qin [30,32], Reiser [31], and a comprehensive review can be found in courses taught on intense beams by Barnard and Lund [67,68]. An early treatment of the model within the context of self-consistent beam equilibria with space charge was presented by Sacherer [86]. Here we parallel the formulation presented by Barnard and Lund to review general properties of the continuous-focusing model for later application in formulating approximate Vlasov loads for focusing channels with $s$-varying applied-focusing forces that improve on the KV model. Details of specific choices of continuousfocusing beam equilibria are presented in Appendices D, $\mathrm{E}$, and F. In a continuous-focusing channel, $\kappa_{x}=\kappa_{y}=$ $k_{\beta 0}^{2}=$ const and the transverse particle Hamiltonian $H_{\perp}$ given by Eq. (8) is a single-particle constant of the motion with $H_{\perp}=$ const. Therefore, any function

$$
f_{\perp}\left(\mathbf{x}_{\perp}, \mathbf{x}_{\perp}^{\prime}, s\right)=f_{\perp}\left(H_{\perp}\right)
$$

satisfying $f_{\perp} \geq 0$ at $s=s_{i}$ will form a valid stationary $(\partial / \partial s=0)$ equilibrium solution to the Vlasov equation (7) with continuous focusing. Moreover, functional bounds can be employed to show that the monotonicity condition $\partial f_{\perp}\left(H_{\perp}\right) / \partial H_{\perp} \leq 0$ is a sufficient condition for stability of the continuous-focusing equilibrium $f_{\perp}$ to both small- and large-amplitude perturbations [30,32,87-89]. Conversely, any continuous-focusing equilibrium not satisfying $\partial f_{\perp}\left(H_{\perp}\right) / \partial H_{\perp} \leq 0$ meets a necessary condition for instability and intuitively one expects that such nonmonotonic profiles to have "free energy" to drive instabilities.

It can be shown [90] that any valid choice of function $f_{\perp}\left(H_{\perp}\right)$ with $\partial f_{\perp}\left(H_{\perp}\right) / \partial H_{\perp} \leq 0$ necessarily produces an axisymmetric $(\partial / \partial \theta=0)$ continuous-focusing equilibrium when the aperture is axisymmetric or sufficiently large to have a negligible effect on the beam (as will be assumed to hold in the remainder of this section). In this case, the Poisson equation (9) can be expressed as

$$
\frac{1}{r} \frac{\partial}{\partial r}\left(r \frac{\partial \phi}{\partial r}\right)=-\frac{q n}{\epsilon_{0}}=-\frac{q}{\epsilon_{0}} \int d^{2} x_{\perp}^{\prime} f_{\perp}\left(H_{\perp}\right),
$$

where $r=\sqrt{x^{2}+y^{2}}$ is the transverse radial coordinate. It is convenient to define an effective potential [30-32,91]

$$
\psi(r) \equiv \frac{1}{2} k_{\beta 0}^{2} r^{2}+\frac{q \phi(r)}{m \gamma_{b}^{3} \beta_{b}^{2} c^{2}} .
$$

Then,

$$
H_{\perp}=\frac{1}{2} \mathbf{x}_{\perp}^{\prime 2}+\psi
$$

and system axisymmetry can be exploited to calculate the beam density as

$$
n(r)=\int d^{2} x_{\perp}^{\prime} f_{\perp}\left(H_{\perp}\right)=2 \pi \int_{\psi}^{\infty} d H_{\perp} f_{\perp}\left(H_{\perp}\right),
$$

to recast the Poisson equation (33) as

$$
\frac{1}{r} \frac{\partial}{\partial r}\left(r \frac{\partial \psi}{\partial r}\right)=2 k_{\beta 0}^{2}-\frac{2 \pi q^{2}}{m \epsilon_{0} \gamma_{b}^{3} \beta_{b}^{2} c^{2}} \int_{\psi}^{\infty} d H_{\perp} f_{\perp}\left(H_{\perp}\right) .
$$

An explicit solution of this equation for $\psi$ [or equivalently Eq. (33) for $\phi$ ] is necessary to calculate the continuousfocusing equilibrium density profile $n=\int d^{2} x_{\perp}^{\prime} f_{\perp}\left(H_{\perp}\right)$. For most physically appealing, smooth choices of $f_{\perp}\left(H_{\perp}\right)$ the equation is highly nonlinear and the solution must be done numerically. Details on how the solution is best carried out vary with the choice of $f_{\perp}\left(H_{\perp}\right)$. In some cases it can be advantageous to eliminate $\psi$ in Eq. (37) in terms of the density $n$ using Eq. (36).

To better understand the continuous-focusing equilibrium structure, it can be useful to calculate the radial kinetic temperature profile $T_{x}=\left\langle x^{\prime 2}\right\rangle_{\mathbf{x}_{\perp}^{\prime}}$ [see Eq. (14)] of the axisymmetric equilibrium defined by $f_{\perp}\left(H_{\perp}\right)$. By symmetry, $T_{x}=T_{y}$. Using Eq. (35), explicit calculation of $T_{x}(r)$ can be simplified as 


$$
\begin{aligned}
T_{x}(r) n(r) & =\frac{1}{2} \int d^{2} x_{\perp}^{\prime} \mathbf{x}_{\perp}^{\prime 2} f_{\perp}\left(H_{\perp}\right) \\
& =2 \pi \int_{\psi}^{\infty} d H_{\perp}\left(H_{\perp}-\psi\right) f_{\perp}\left(H_{\perp}\right) .
\end{aligned}
$$

The axisymmetric continuous-focusing equilibrium beam formed by $f_{\perp}\left(H_{\perp}\right)$ will be envelope matched to the continuous-focusing channel with $r_{j} \equiv r_{b}=\sqrt{2\left\langle r^{2}\right\rangle_{\perp}}=$ const satisfying the rms-envelope equation

$$
k_{\beta 0}^{2} r_{b}-\frac{Q}{r_{b}}-\frac{\varepsilon_{b}^{2}}{r_{b}^{3}}=0 .
$$

Here, $f_{\perp}\left(H_{\perp}\right)$ can be employed to consistently calculate the statistical beam envelope radius $r_{b}$ [see Eq. (23)] as

$$
r_{b}^{2}=2\left\langle r^{2}\right\rangle_{\perp}=\frac{\int_{0}^{\infty} d r r^{3} \int_{\psi}^{\infty} d H_{\perp} f_{\perp}\left(H_{\perp}\right)}{\int_{0}^{\infty} d r r \int_{\psi}^{\infty} d H_{\perp} f_{\perp}\left(H_{\perp}\right)},
$$

the line-charge density $\lambda=$ const [see Eq. (10)], or perveance $Q=q \lambda /\left(2 \pi \epsilon_{0} m c^{2} \gamma_{b}^{3} \beta_{b}^{2}\right)$ [see Eq. (22)] as

$$
\lambda=(2 \pi)^{2} q \int_{0}^{\infty} d r r \int_{\psi}^{\infty} d H_{\perp} f_{\perp}\left(H_{\perp}\right),
$$

and the rms-edge emittance $\varepsilon_{j} \equiv \varepsilon_{b}=$ const [see Eq. (24)] as

$$
\begin{aligned}
\varepsilon_{b}^{2} & =2 r_{b}^{2}\left\langle\mathbf{x}_{\perp}^{\prime 2}\right\rangle_{\perp} \\
& =2 r_{b}^{2} \frac{\int_{0}^{\infty} d r r \int_{\psi}^{\infty} d H_{\perp}\left(H_{\perp}-\psi\right) f_{\perp}\left(H_{\perp}\right)}{\int_{0}^{\infty} d r r \int_{\psi}^{\infty} d H_{\perp} f_{\perp}\left(H_{\perp}\right)} .
\end{aligned}
$$

Comparing Eqs. (38) and (42) and employing previous results, note that the beam emittance $\varepsilon_{b}$ is related to the spatially averaged radial temperature profile of the beam by

$$
\varepsilon_{b}^{2}=2 r_{b}^{2} \frac{\int_{0}^{\infty} d r r T_{x}(r) n(r)}{\int_{0}^{\infty} d r r n(r)} .
$$

Although the self-field potential specified by Eq. (37) is generally difficult to solve for physically appealing, smooth choices of $f_{\perp}\left(H_{\perp}\right)$, some general comments can be made on the solution structure. For $f_{\perp}\left(H_{\perp}\right)$ without singularities, one expects a radial density profile $n(r)=$ $\int d^{2} x_{\perp}^{\prime} f_{\perp}\left(H_{\perp}\right)$ that, for strong space charge, becomes very flat in the core due to Debye screening [30] before falling off near the beam edge with a radial shape characteristic of the form of $f_{\perp}\left(H_{\perp}\right)$. For weak space charge the characteristic radial edge shape will reach more deeply into the core of the beam. Screening effects associated with strong space charge will also influence the kinetic temperature profile $T_{x}(r)$ of the continuous-focusing distribution. For very strong space charge, screening can cause the equilibrium density profile to become flat enough where special numerical methods prove necessary to solve the equilibrium Poisson equation for $\phi$. Also, as a practical matter, parameters used in the definition of $f_{\perp}\left(H_{\perp}\right)$ [e.g., thermal equilibrium: $f_{\perp}\left(H_{\perp}\right)=C_{1} \exp \left(-C_{2} H_{\perp}\right)$, where $C_{1}=$ const and $C_{2}=$ const are positive parameters; see Appendix F] should be identified in terms of parameters customarily employed in beam physics to facilitate application of results. Parameters should be related to particle properties (i.e., the charge $q$, mass $m$, and relativistic factors $\beta_{b}$ and $\left.\gamma_{b}\right)$, the applied-focusing strength $\left(k_{\beta 0}^{2}\right)$, the perveance $(Q)$, and the rms-edge emittance $\left(\varepsilon_{b}\right)$. This typically requires analysis of highly nonlinear integral constraint equations that must be solved consistently with changes in the equilibrium potential $\phi$ as parameters used in the definition of $f_{\perp}\left(H_{\perp}\right)$ vary. Concrete examples of such procedures are given in Appendices D, E, and F for unit-step ("waterbag" distribution), linear ("parabolic" distribution), and Gaussian ("thermal" distribution) choices of $f_{\perp}\left(H_{\perp}\right)$. These three radically different choices of $f_{\perp}\left(H_{\perp}\right)$ functions serve to illustrate the commonality and differences involved with a wide range of choice in equilibrium distributions. All three of these choices satisfy $\partial f_{\perp}\left(H_{\perp}\right) / \partial H_{\perp} \leq 0$ and therefore correspond to stable Vlasov equilibria.

It is instructive to examine the form of the KV equilibrium distribution presented in Sec. III A in the ideal, continuous-focusing limit. In this case, $r_{j}=r_{b}=$ const, $r_{j}^{\prime}=0$, and $\varepsilon_{j}=\varepsilon_{b}=$ const. Using the delta-function property $\delta(C x)=\delta(x) /|C|$ with $C=$ const, the KV distribution (16) can be expressed in the continuous limit as

$$
f_{\perp}=\frac{\lambda}{q \pi^{2} r_{b}^{2}} \delta\left(\frac{1}{2} \mathbf{x}_{\perp}^{\prime 2}+\frac{\varepsilon_{b}^{2}}{2 r_{b}^{4}} \mathbf{x}_{\perp}^{2}-\frac{\varepsilon_{b}^{2}}{2 r_{b}^{2}}\right) .
$$

For the uniform-density, axisymmetric beam core of the continuous focused KV beam, the Poisson equation (33) can be integrated to show that the single-particle Hamiltonian [see Eq. (8)] is expressible as

$$
H_{\perp}=\frac{1}{2} \mathbf{x}_{\perp}^{\prime 2}+\frac{\varepsilon_{b}^{2}}{2 r_{b}^{4}} \mathbf{x}_{\perp}^{2}+\text { const. }
$$

At the beam edge $\left(r=r_{b}\right)$, all particles must turn, $\mathbf{x}_{\perp}^{\prime}=0$, and $\left.H_{\perp}\right|_{r=r_{b}}=\varepsilon_{b}^{2} /\left(2 r_{b}^{2}\right)+$ const $\equiv H_{b}$. Using these results, the continuous-focusing KV distribution (44) can be equivalently expressed as

$$
f_{\perp}=\frac{\lambda}{2 \pi^{2} q r_{b}^{2}} \delta\left(H_{\perp}-H_{b}\right)
$$

Using $\lambda=q \hat{n} \pi r_{b}^{2}$ with $\hat{n}=$ const the density within the beam, Eq. (46) can be written as $f_{\perp}=\frac{\hat{n}}{2 \pi} \delta\left(H_{\perp}-H_{b}\right)$. This alternative expression for the KV distribution function in a continuous-focusing channel is commonly employed in the literature [30-32,67,68]. The equivalence of Eqs. (16) and (46) shows that for continuous focusing, the Courant-Snyder invariant form of the KV distribution reduces to the expected Hamiltonian invariant form. This should not be interpreted as implying that the CourantSnyder invariant is equivalent to $H_{\perp}=$ const for other than 
continuous focusing. In periodic-focusing channels the Hamiltonian $H_{\perp}$ has explicit $s$ dependence from the applied-focusing terms. Unfortunately, this $s$ dependence renders $H_{\perp}$ inappropriate for constructing single-particle invariants and equilibrium distribution functions for focusing channels with $s$-varying focusing forces. It is also interesting to point out that $\partial f_{\perp}\left(H_{\perp}\right) / \partial H_{\perp}$ changes sign about $H_{\perp}=H_{b}$, showing that the $\mathrm{KV}$ distribution does not satisfy the sufficient condition for stability and therefore satisfies a necessary condition for instability. Well-known kinetic analyses show that the continuous-focusing $\mathrm{KV}$ distribution is unstable for all space-charge strengths satisfying $\sigma / \sigma_{0}<0.3985[38,42]$.

The KV model can be applied in an rms-equivalent beam sense to characterize relative space-charge strength of a continuous-focusing equilibrium with nonuniform $n(r)$. Taking the equivalent axial "lattice period" length of phase accumulation to be $L_{p}$, we first set $k_{\beta 0}=\sigma_{0} / L_{p}$, where $\sigma_{0}$ is the undepressed particle phase advance over $L_{p}$. Then the depressed phase-advance equation (25) for $\sigma_{j} \equiv \sigma$ is applied over an axial length $L_{p}$ with $\varepsilon_{j}=\varepsilon_{b}$ and $r_{j}=r_{b}$ given by the matched-beam envelope equation (39). This yields

$$
\frac{\sigma}{\sigma_{0}}=\sqrt{1-\frac{Q}{k_{\beta 0}^{2} r_{b}^{2}}}=\frac{\varepsilon_{b}}{k_{\beta 0} r_{b}^{2}} .
$$

In this context, $\sigma / \sigma_{0}$ characterizes the relative spacecharge strength of an equilibrium with $\sigma / \sigma_{0}=1(Q=$ $0)$ corresponding to negligible space-charge strength, and $\sigma / \sigma_{0}=0\left(\varepsilon_{b}=0\right)$ corresponding to an equilibrium with maximum space-charge strength.

Some authors employ alternative dimensionless parameters to $\sigma / \sigma_{0}$ when characterizing relative space-charge strength. One choice [32] is to define a normalized selffield intensity parameter $s_{b}$ as the ratio of one-half of the squared relativistic plasma frequency formed from the onaxis $(r=0)$ density $\hat{n}=n(r=0)$ of the distribution [i.e., $\hat{\omega}_{p}^{2} /\left(2 \gamma_{b}\right)$ with $\left.\hat{\omega}_{p}^{2}=q^{2} \hat{n} /\left(m \epsilon_{0}\right)\right]$ to the squared frequency of single-particle oscillations in the applied-focusing field $\left[\gamma_{b}^{2} \beta_{b}^{2} c^{2} k_{\beta 0}^{2}\right]$, i.e.,

$$
s_{b} \equiv \frac{\hat{\omega}_{p}^{2}}{2 \gamma_{b}^{3} \beta_{b}^{2} c^{2} k_{\beta 0}^{2}} .
$$

For a KV continuously focused beam, $s_{b}$ and $\sigma / \sigma_{0}$ are simply related by

$$
s_{b}=1-\left(\frac{\sigma}{\sigma_{0}}\right)^{2} .
$$

The dimensionless self-field parameter $s_{b}$ defined in terms of the on-axis density of the beam can be algebraically convenient when specifying continuous-focusing equilibria with monotonic radial density profiles $n(r)$ satisfying $\partial n(r) / \partial r \leq 0$. However, monotonic equilibrium specifications of $f_{\perp}\left(H_{\perp}\right)$ with $\partial f_{\perp}\left(H_{\perp}\right) / \partial H_{\perp} \leq 0$ (see examples in Appendices D, E, and F) generally result in $s_{b} \simeq 1$ over a relatively large range of high space-charge intensity (with rms equivalent $\sigma / \sigma_{0} \lesssim 1 / 2$ ), rendering $s_{b}$ a relatively insensitive measure in regimes where $s_{b} \rightarrow 1$.

The form of an continuous-focusing equilibrium distribution $f_{\perp}\left(H_{\perp}\right)$ and the corresponding density $n(r)$ are strongly linked. For monotonic density profiles with $\partial n(r) / \partial r \leq 0, \hat{n}=n(r=0)$ is maximum value of $n(r)$ and the density inversion theorem $[30,32,67,68,92]$ can be employed to calculate the equilibrium function $f_{\perp}\left(H_{\perp}\right)$ from a specified density profile as

$$
f_{\perp}\left(H_{\perp}\right)=-\left.\frac{1}{2 \pi} \frac{\partial n}{\partial \psi}\right|_{\psi=H_{\perp}} .
$$

If $f_{\perp}\left(H_{\perp}\right)$ satisfies $\partial f_{\perp}\left(H_{\perp}\right) / \partial H_{\perp} \leq 0$, then the equilibrium specified by $n(r)$ will be stable.

Although the structure of the continuous-focusing equilibria satisfying $\partial f_{\perp}\left(H_{\perp}\right) / \partial H_{\perp} \leq 0$ is physically appealing and stable, unfortunately the continuous-focusing model cannot provide a direct guide for properties of beam transport in realistic $s$-varying applied-focusing channels. The continuous-focusing function $\kappa_{j}=k_{\beta 0}^{2}=$ const is equivalent to a partially neutralizing, immobile $(m \rightarrow \infty)$ background species with charge density $\rho=$ $-2 m \epsilon_{0} \gamma_{b} \beta_{b}^{2} c^{2} k_{\beta 0}^{2} / q=$ const and can only represent realistic, $s$-varying focusing forces in an average sense. While this approximate continuous and $s$-varying focusing correspondence can be useful in simplified estimates of transport properties where the system is far from instability, periodic-focusing channels have well-known instabilities that are not present in the continuous-focusing limit [16,33-35]. Continuous-focusing analogies to periodicfocusing systems typically become progressively worse as the strength of the applied-focusing field (as measured by $\sigma_{0}$ ) increases-particularly for alternating-gradient quadrupole focusing. The desire for radially compact beams often requires applications with high focusing strength, exacerbating this breakdown of approximate analogies. Canonical transforms [30,45,67,68] and Hamiltonian average theories [32,52-58] can improve applicability of the continuous-focusing model to systems with nonconstant (especially periodic) focusing-particularly as the focusing becomes weaker, e.g., for $\sigma_{0} \lesssim 60^{\circ}$ in periodic lattices.

To load the continuous-focusing distributions (32) in direct Vlasov or PIC simulations, parameters used in defining the function $f_{\perp}\left(H_{\perp}\right)$ should be determined in terms of standard beam parameters (i.e., $k_{\beta 0}^{2}, Q, \varepsilon_{b}$, etc.). Such procedures are generally nontrivial, as evident from the examples in Appendices D, E, and F. With equilibrium parameters specified, the effective potential $\psi$ must then be calculated (generally numerically) within the beam to determine $H_{\perp}=\mathbf{x}_{\perp}^{\prime 2} / 2+\psi$ and thereby fully specify $f_{\perp}\left(H_{\perp}\right)$. For direct Vlasov simulations $f_{\perp}\left(\mathbf{x}_{\perp}^{\prime 2} / 2+\psi\right)$ can then be loaded on the $4 \mathrm{D}$ (or $3 \mathrm{D} / 2 \mathrm{D}$ if axisymmetry 
is partially/fully taken into account) phase-space grid of the simulation.

For PIC simulations, macroparticles can be loaded to approximate the continuous-focusing distributions by building on techniques discussed in Sec. III A for initializing macroparticles to model the KV distribution. First, the radial beam density profile $n(r)$ can be calculated from Eq. (36) using the calculated radial effective potential $\psi(r)$. Then macroparticle spatial coordinates $\mathbf{x}_{\perp}$ can be loaded consistent with this density profile using a probability transform to map a uniform distribution within a unit circle to a distribution consistent with the radial density profile $n(r)$. This can be accomplished using two independent, uniformly distributed random numbers $\hat{u}_{r} \in[0,1)$ and $\hat{u}_{\theta} \in[0,1)$ for each macroparticle, carrying out a probability transformation [12]

$$
\frac{\int_{0}^{r} d \tilde{r} \tilde{r} n(\tilde{r})}{\int_{0}^{\infty} d r r n(r)}=\hat{u}_{r},
$$

and taking

$$
x=r\left(\hat{u}_{r}\right) \cos \left(2 \pi \hat{u}_{\theta}\right), \quad y=r\left(\hat{u}_{r}\right) \sin \left(2 \pi \hat{u}_{\theta}\right) .
$$

Here, $r\left(\hat{u}_{r}\right)$ is the smallest positive solution of Eq. (51), and $\hat{u}_{\theta}$ generates a uniform distribution of azimuthal coordinate angles in the axisymmetric beam. If a lowerdimensional simulation is used (with "ring" macroparticles) to more efficiently model the axisymmetric beam, then only macroparticle radii $r\left(\hat{u}_{r}\right)$ need be calculated. The solution $r\left(\hat{u}_{r}\right)$ must, in general, be solved numerically for smooth $n(r)$. When a large number of macroparticles must be loaded, the transform in Eq. (51) can be calculated for gridded values of $\hat{u}_{r} \in[0,1]$ and the corresponding gridded values of $r\left(\hat{u}_{r}\right)$ stored to allow efficient calculation of $r\left(\hat{u}_{r}\right)$ for arbitrary values of $\hat{u}_{r} \in[0,1)$ by calculating the nearest grid indices and interpolating. For cases where $n(r)$ does not have a sharp edge, the grid will generally need to be cutoff at a radius beyond which the density is negligible. If a uniform grid is employed the cutoff should not be chosen too large or the resolution in the core of the distribution will be degraded (see, for example, the thermal equilibrium analysis in Appendices F and G). Some simple classes of density profiles allow analytical solution. For example, if the density is uniform within radius $r=r_{e}$, then Eq. (51) yields $r\left(\hat{u}_{r}\right)=r_{e} \sqrt{\hat{u}_{r}}$ [compare this to Eq. (27) with $r_{x}=r_{y}=r_{e}$ ].

With the macroparticle coordinates loaded and the radial dependence of $\psi(r)$ thereby specified, the macroparticle angles $\mathbf{x}_{\perp}^{\prime}$ can be generated consistently with $f_{\perp}\left(H_{\perp}\right)$ using an analogous procedure to the one employed for the coordinate loading. Taking $H_{\perp}=U+\psi$ with $U=$ $\mathbf{x}_{\perp}^{12} / 2$, the probability transform

$$
\frac{\int_{0}^{U} d \tilde{U} f_{\perp}(\tilde{U}+\psi)}{\int_{0}^{\infty} d U f_{\perp}(U+\psi)}=\hat{u}_{U}
$$

is solved for $U\left(\hat{u}_{U}\right)$ and then the angles are set consistent with beam axisymmetry using

$$
\begin{aligned}
& x^{\prime}=\sqrt{2 U\left(\hat{u}_{U}\right)} \cos \left(2 \pi \hat{u}_{\varphi}\right), \\
& y^{\prime}=\sqrt{2 U\left(\hat{u}_{U}\right)} \sin \left(2 \pi \hat{u}_{\varphi}\right) .
\end{aligned}
$$

Here, $\hat{u}_{U} \in[0,1)$ and $\hat{u}_{\varphi} \in[0,1)$ are two independent, uniformly distributed random numbers generated for each macroparticle. In general, $U\left(\hat{u}_{U}\right)$ must be calculated numerically. Analogous to the case for the macroparticle coordinates discussed above, values of the transform in Eq. (53) can be precalculated on a grid and interpolation applied to efficiently load a large number of macroparticles. In simulations where beam axisymmetry is fully exploited, only $r^{\prime}$ may be necessary to initialize macroparticles. In this case, using $r^{\prime}=\left(x x^{\prime}+y y^{\prime}\right) / r$ and Eqs. (52) and (54), it follows that $r^{\prime}$ can be loaded as

$$
r^{\prime}=\sqrt{2 U\left(\hat{u}_{U}\right)} \cos \left(2 \pi \hat{u}_{r^{\prime}}\right)
$$

with $\hat{u}_{r^{\prime}} \in[0,1)$ a uniformly distributed random number for each macroparticle.

Analogous to the situation discussed for the KV distribution in Sec. III A, the random numbers employed to load the continuous-focusing distribution $\left\{\hat{u}_{r}, \hat{u}_{\theta}\right\}$ and/or $\left\{\hat{u}_{U}, \hat{u}_{\varphi}\right\}$ can be replaced by ordered sets of numbers to reduce initial statistical noise for simulations with a finite number of macroparticles.

It is interesting to point out that the loading formalism outlined above can be applied for a specified, stable monotonic decreasing radial density profile $n(r)$ without detailed knowledge of the corresponding equilibrium function $f_{\perp}\left(H_{\perp}\right)$ that specifies the continuous-focusing distribution. First, macroparticle coordinates $\mathbf{x}_{\perp}$ consistent with $n(r)$ can be calculated from Eqs. (51) and (54). The equilibrium potential $\psi$ can then be calculated from Eqs. (36) and (37), and this result applied in the inversion theorem (50) to implicitly specify $f\left(H_{\perp}\right)$ for use in Eqs. (53) and (54) to load the macroparticle angles $\mathbf{x}_{\perp}$.

Because the continuous-focusing distributions are exact Vlasov equilibria, any evolution in simulated distribution from the loaded beam results from numerical approximations in the procedure used to load the distribution and/or in the Vlasov simulations. This property, when employing accurate loads, can render the continuous-focusing distributions useful for checking the accuracy of simulations. Two-dimensional PIC slice simulations illustrating this point are shown in Fig. 2 for a waterbag (step-function) choice of $f\left(H_{\perp}\right)$. Properties of the waterbag equilibrium are analyzed in detail in Appendix D. Codes and parameter choices made in the simulations are described in Sec. II. Simulations illustrated were carried out of strong relative space-charge strength $\left(\sigma / \sigma_{0}=0.2\right)$, so the equilibrium radial density profile is flat in the core of the beam. The waterbag equilibrium has a sharp edge in phase-space projections, which can aid visualization of small evolutions 

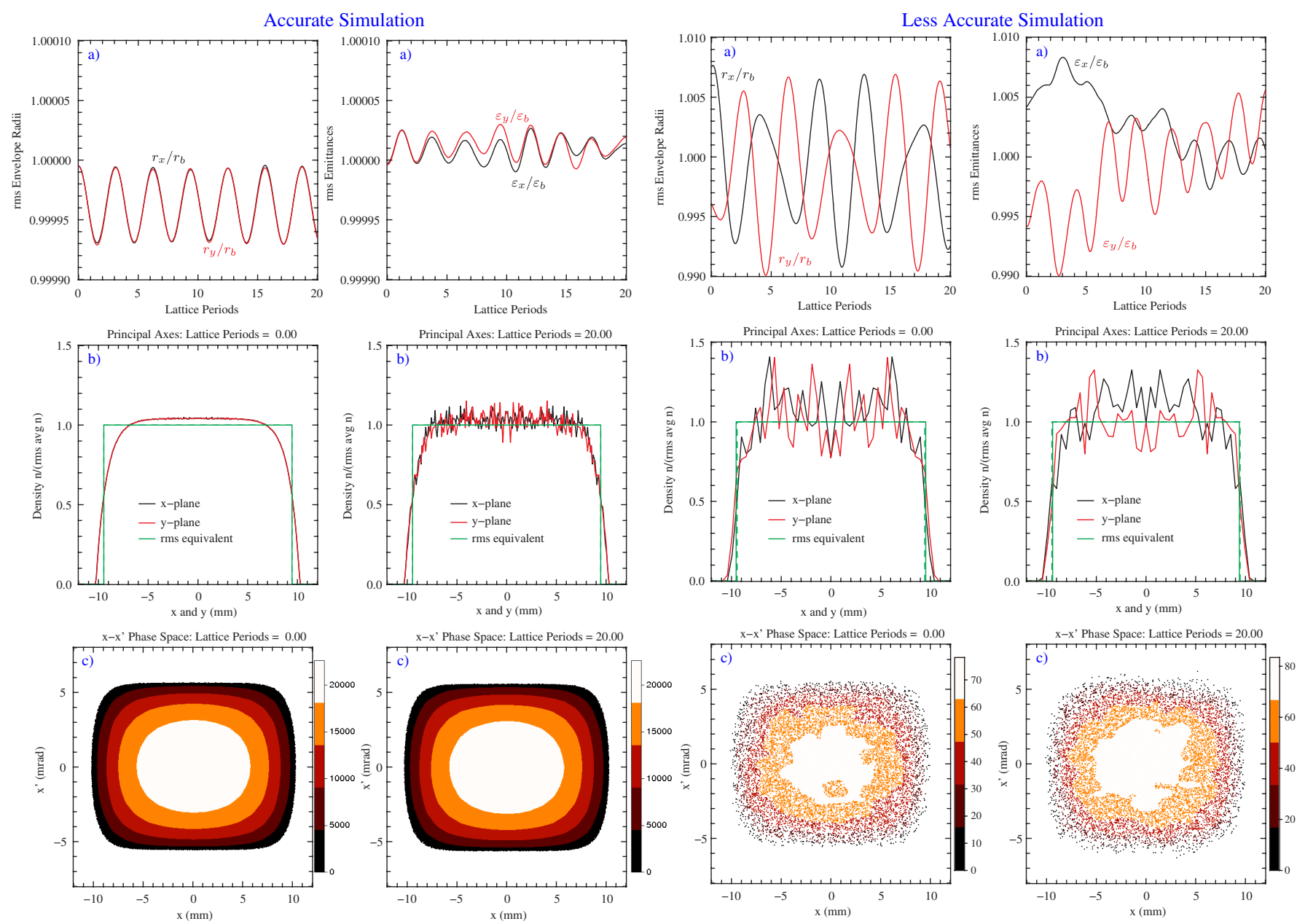

FIG. 2. (Color) PIC simulations of an initial waterbag equilibrium distribution in a continuous-focusing channel with $\sigma_{0}=80^{\circ}$ and $\sigma / \sigma_{0}=0.2$ for a well-converged (two left columns; $N_{r}=100, N_{\mathrm{ppg}}=500$ ) and a less-converged simulation (two right columns: $\left.N_{r}=20, N_{\text {ppg }}=40\right)$. In row (a) the evolutions of rms-envelope radii $\left[r_{j} / r_{j}(s=0)\right]$ and rms-edge emittances $\left[\varepsilon_{j} / \varepsilon_{j}(s=0)\right]$ are shown as a function of lattice periods $\left(s / L_{p}\right)$. In rows (b) and (c), the principal axis beam density profiles and $x$ - $x^{\prime}$ phase-space projections are shown at zero (load) and 20 lattice periods. (WARP simulations with parameters: $N_{p}=3$ and $N_{s}=25$.)

induced by numerical approximations. Both an accurate simulation and a less accurate simulation with poorer resolution and statistics are shown. Details of the waterbag equilibrium distribution are presented in Appendix D and the simulations and parameter choices are described in Sec. II. To precisely load the distribution in both the accurate and less accurate cases, the radial density transformation (51) is solved on a uniform mesh of 500 points and the angle transform (53) is solved exactly (see discussions in Appendix D). In the accurate simulation, ordered digit-reversed numbers are used to generate a load with reduced noise in phase space, whereas the less accurate simulation uses pseudorandom numbers to generate the load resulting enhanced initial statistical noise relative to ordered numbers. Statistical beam envelope radii $r_{j}$ and emittances $\varepsilon_{j}$ are calculated using Eqs. (23) and (24) with centroid measures subtracted [see Eq. (15) and the related discussion]. Quantities associated with the $x$ and $y$ planes are plotted in black and red, respectively. Profiles of the beam density $n$ are shown along the $x$ and $y$ axes (in black and red, respectively) as calculated from the gridded charge density in the simulation with no additional smoothing. The density is normalized by the rms average measure, $\lambda /\left(q \pi r_{x} r_{y}\right)$, so values not equal to unity indicate deviations from an rms-equivalent KV beam. Density profile plots are superimposed with the density of an rmsequivalent $\mathrm{KV}$ beam (in green, with unit density as normalized). The $x-x^{\prime}$ phase-space projections are generated by plotting macroparticle markers that are color coded based on the local phase-space density. All macroparticles are shown in the projections of the less accurate simulation, whereas a method is employed in the plots of the accurate simulation that shows almost all particles in the lowdensity regions and a sampling of macroparticles in the high-density regions.

The accurate simulation shown in Fig. 2 shows very small evolution in all distribution projections and moments, whereas the less accurate simulation shows a larger, 
but still relatively modest degree of evolution due primarily to poor grid resolution and macroparticle statistics (note the large change in scale between the plots). Fluctuations and oscillations associated with the less accurate simulation are still modest considering the coarse numerical parameters employed. This surprisingly benign consequence of errors likely results from both the stability of the underlying steady equilibrium and that the numerical errors seed a broad spectrum of oscillations that remain bounded by initial conditions (system energy is conserved in continuous focusing) and phase mix. Differences between the $x$ - and $y$-plane envelope and emittance evolutions as well as nonaxisymmetries in the distribution projections that are clearly evident in the less accurate simulation are related to finite statistics and discretizations breaking ideal symmetries. The initial fluctuations in the density profiles of the accurate simulation are suppressed both by the use of large numbers of macroparticles and by the use of ordered numbers in the load. By the end of the evolution, the statistical noise of the accurate simulation has increased to levels expected if pseudorandom numbers had been employed in the load. It appears that the primary error source in the accurate simulation is the discretization associated with the radial mesh introducing a systematic error in the location of the beam edge which primarily translates into a small amplitude breathing mismatch as evident from the regular, nearly in-phase oscillations of the envelope radii $r_{j}$.

\section{Nonequilibrium semi-Gaussian distribution}

The semi-Gaussian distribution $[47,67,68]$ can be defined by

$$
\begin{aligned}
f_{\perp}\left(\mathbf{x}_{\perp}, \mathbf{x}^{\prime}\right)= & \frac{2 \lambda}{q \pi^{2} \varepsilon_{x} \varepsilon_{y}} \Theta\left[1-\left(\frac{x^{2}}{r_{x}^{2}}+\frac{y^{2}}{r_{y}^{2}}\right)\right] \\
& \times \exp \left[-2\left(\frac{r_{x} x^{\prime}-r_{x}^{\prime} x}{\varepsilon_{x}}\right)^{2}-2\left(\frac{r_{y} y^{\prime}-r_{y}^{\prime} y}{\varepsilon_{y}}\right)^{2}\right] .
\end{aligned}
$$

Here,

$$
\Theta(x)= \begin{cases}1, & x>0 \\ 0, & x<0\end{cases}
$$

is a Heaviside unit-step function, $r_{j}$ and $r_{j}^{\prime}(j=x, y)$ are the initial $\left(s=s_{i}\right)$ beam envelope radii and angles, and $\varepsilon_{j}$ are the initial rms-edge emittances. As for the KV distribution, the density $n=\int d^{2} x_{\perp}^{\prime} f_{\perp}$ of the initial distribution is uniform within an ellipse of radii $r_{j}$ as given by Eq. (17). Likewise, the coherent flows $\left\langle x^{\prime}\right\rangle_{\mathbf{x}_{\perp}^{\prime}}$ and $\left\langle y^{\prime}\right\rangle_{\mathbf{x}_{\perp}^{\prime}}$ are identical to the KV expression in Eq. (19). In contrast to the KV distribution, the incoherent angular spreads in $x^{\prime}$ and $y^{\prime}$ are spatially uniform and Gaussian distributed. Direct calculation with Eqs. (14) and (56) shows that the kinetic temperatures corresponding to the Gaussian-distributed spreads are

$$
T_{j}= \begin{cases}\frac{\varepsilon_{j}^{2}}{4 r_{j}^{2}}, & \text { if }\left(x / r_{x}\right)^{2}+\left(y / r_{y}\right)^{2}<1, \\ 0, & \text { otherwise }\end{cases}
$$

The semi-Gaussian distribution is not an equilibrium of the Vlasov equation (7) for a linear-focusing channel with finite beam space charge. The distribution will evolve from the initial condition, resulting in a change in form associated with the launching of a transient, nonlinear wave $[48,91]$. This wave evolution will result in rmsedge emittances evolving from the initial conditions $\left(\varepsilon_{j} \neq\right.$ const) and the subsequent envelope evolution $r_{j}(s)$ will only approximately follow that of the KV envelope equation (21).

The strength of the transient evolution of the semiGaussian distribution depends primarily on the relative intensity of the applied-focusing and space-charge forces. The initially uniform space charge within an elliptical beam envelope gives linear electrostatic defocusing space-charge forces when image-charge effects are negligible. But the initially uniform temperature within the beam results in an unbalanced thermal force inconsistent with the sharp beam edge. The collective wave launched is a manifestation of this inconsistency. Despite this nonequilibrium structure, the semi-Gaussian distribution is commonly employed to model space-charge-dominated beams where Debye screening is expected to lead to a flat density profile for a relaxed distribution. The semi-Gaussian distribution has manifest rms equivalency with the KV distribution which simplifies interpretation, and the distribution structure corresponds roughly to a beam that would be produced by an ideal injector (uniform current density within the beam emitted from a diode with ChildLangmuir emission $[31,93]$ and spatially uniform temperature due to a heated source at local thermodynamic equilibrium). Waves launched by the nonequilibrium form of the semi-Gaussian distribution generally lead to small, space-charge intensity dependent reductions in the rmsedge emittance where the beam is stable $[31,83,91]$. Stability is considered in the sense of having limited wave growth from the initial transient evolution. In the case of stability, transient-wave perturbations launched from the initial semi-Gaussian distribution rapidly wash out due to phase mixing and nonlinear collective effects present for finite space charge $[15,48,49,91]$. SemiGaussian distributions with $\varepsilon_{x} \neq \varepsilon_{y}$ (i.e., $T_{x} \neq T_{y}$ ) sufficiently anisotropic can lead to evolutions with characteristics of space-charge driven instabilities in situations where the system drives to a more thermally isotropic state [94].

Loading of the initial semi-Gaussian distribution (56) can be carried out using similar steps to those described for the KV distribution in Sec. III A. For direct Vlasov simulations, loading $f_{\perp}$ on the phase-space grid is generally less 
challenging than for a KV distribution because the semiGaussian distribution is not singular in 4D phase space. The most significant issue is the need to adequately represent the sharp elliptical beam edge in $\mathbf{x}_{\perp}$ on the phasespace grid and to have sufficient resolution in the phasespace mesh to model the nonlinear transient wave launched from the lack of detailed equilibrium force balance $[22,48,49,94]$. For PIC simulations, macroparticle spatial coordinates $\mathbf{x}_{\perp}$ can be loaded exactly as discussed for the KV distribution [see Eq. (27)]. Macroparticle angles $\mathbf{x}_{\perp}^{\prime}$ can be loaded in terms of coherent and incoherent components terms as $\mathbf{x}_{\perp}^{\prime}=\left.\mathbf{x}_{\perp}^{\prime}\right|_{\mathrm{c}}+\left.\mathbf{x}_{\perp}^{\prime}\right|_{\text {ic }}$ [see Eq. (28)] with the coherent term $\mathbf{x}_{\perp}^{\prime} l_{c}$ set exactly as for the KV distribution using Eq. (29), and the incoherent term $\left.\mathbf{x}_{\perp}^{\prime}\right|_{\text {ic }}$ simply set with

$$
\left.x^{\prime}\right|_{\mathrm{ic}}=\frac{\varepsilon_{x}}{2 r_{x}} \hat{g}_{x},\left.\quad y^{\prime}\right|_{\mathrm{ic}}=\frac{\varepsilon_{y}}{2 r_{y}} \hat{g}_{y}
$$

rather than Eq. (31). Here, $\hat{g}_{x}$ and $\hat{g}_{y}$ are two independent, Gaussian-distributed random numbers with unit variance generated for each macroparticle. If bounded particle phase space is necessary, the $\hat{g}_{j}$ can be replaced with truncated, Gaussian-distributed values with minimal error if the unit variance Gaussian is truncated for values beyond a few units. Note the similarity of the incoherent load angles specified by Eq. (59) with the formula used to specify the macroparticle angles for the continuousfocusing thermal equilibrium distribution [see Appendix F, Eq. (F23)].

Analogously to the situation discussed for the KV distribution in Sec. III A, the random numbers employed to load the semi-Gaussian distribution can be replaced by ordered sets of numbers to reduce initial statistical noise for a finite number of macroparticles. Appropriate sets of ordered Gaussian numbers $\left\{\hat{g}_{j}\right\}$ can be generated employing the same transforms used to map a uniformly distributed random number $\hat{u} \in[0,1)$ to a Gaussian-distributed random number $\hat{g}$ with unit variance [95] to ordered sets of $\left\{\hat{u}_{j}\right\}$.

Examples of Vlasov simulations of initial semiGaussian distributions can be found in the literature $[22,48,49]$ and will not be repeated here. The ease of loading the semi-Gaussian distribution together with the relative faithfulness of the distribution to the form expected for a cold beam with strong relative space-charge forces has resulted in the semi-Gaussian distribution being the load of choice in many intense-beam simulation studies. The main disadvantage of the semi-Gaussian load is that the lack of approximate force balance near the edge of the beam launches a strong transient wave that can complicate interpretations of other effects of interest.

\section{Nonequilibrium distributions of linear-field Courant-Snyder invariants}

An alternative nonequilibrium distribution that is a specified function of linear-field single-particle CourantSnyder invariants of an rms-equivalent beam has been formulated by Batygin [50,51] building on earlier work $[31,39]$. Here we review results under a common notation to aid comparisons to other classes of initial distribution functions. As with the KV (see Sec. III A) and semiGaussian (see Sec. IIIC) distributions, these distributions have elliptical symmetry and consequently can employed in a linear-focusing channel with $s$ variation in the focusing functions $\kappa_{j}(j=x, y)$.

The linear-field Courant-Snyder invariant (LCS) distribution is specified as

$$
f_{\perp}\left(\mathbf{x}_{\perp}, \mathbf{x}_{\perp}^{\prime}, s\right)=\frac{\lambda}{q} f\left(\mathcal{A}^{2}\right),
$$

where $f\left(\mathcal{A}^{2}\right)$ is any function of the single-particle amplitude

$$
\mathcal{A}^{2} \equiv\left(\frac{x}{r_{x}}\right)^{2}+\left(\frac{r_{x} x^{\prime}-r_{x}^{\prime} x}{\varepsilon_{x}}\right)^{2}+\left(\frac{y}{r_{y}}\right)^{2}+\left(\frac{r_{y} y^{\prime}-r_{y}^{\prime} y}{\varepsilon_{y}}\right)^{2},
$$

with $f \geq 0$ that satisfies the normalization constraint

$$
\int d^{2} x_{\perp} \int d^{2} x_{\perp}^{\prime} f\left(\mathcal{A}^{2}\right)=1,
$$

and the moment constraint

$$
\frac{\int_{0}^{\infty} d U U G(U)}{\int_{0}^{\infty} d U G(U)}=\frac{1}{2},
$$

with

$$
G(U) \equiv \int_{U}^{\infty} d \tilde{U} f(\tilde{U})
$$

The quantities $r_{j}$ and $\varepsilon_{j}$ employed in $\mathcal{A}^{2}$ are the statistical envelope radii and emittances of the distribution as defined by Eqs. (23) and (24) in an rms-equivalent beam sense (see Sec. III A). The form of $\mathcal{A}^{2}$ and the normalization and moment constraints are sufficient to ensure that the LCS distribution defined by Eqs. (60)-(64) satisfies rms equivalency for arbitrary (physical) values of $r_{j}, r_{j}^{\prime}$, and $\varepsilon_{j}$. This rms equivalency is demonstrated in Appendix $\mathrm{C}$ through the use of canonical transformations.

The amplitude $\mathcal{A}^{2}$ can be resolved as

$$
\mathcal{A}^{2}=\mathcal{A}_{x}^{2}+\mathcal{A}_{y}^{2}
$$

where 


$$
\begin{aligned}
& \mathcal{A}_{x}^{2} \equiv\left(\frac{x}{r_{x}}\right)^{2}+\left(\frac{r_{x} x^{\prime}-r_{x}^{\prime} x}{\varepsilon_{x}}\right)^{2}, \\
& \mathcal{A}_{y}^{2} \equiv\left(\frac{y}{r_{y}}\right)^{2}+\left(\frac{r_{y} y^{\prime}-r_{y}^{\prime} y}{\varepsilon_{y}}\right)^{2} .
\end{aligned}
$$

The amplitudes $\mathcal{A}_{j}^{2}$ are single-particle $j$-plane CourantSnyder invariants of a particle evolving within the linear fields (including applied and space charge) of an rmsequivalent beam (see Appendix B). Consequently, the LCS distribution $f_{\perp} \propto f\left(\mathcal{A}^{2}\right)=f\left(\mathcal{A}_{x}^{2}+\mathcal{A}_{y}^{2}\right)$ is constant on two-dimensional elliptical surfaces in $x$ - $x^{\prime}$ and $y-y^{\prime}$ phase space where $\mathcal{A}_{j}^{2}=$ const as well as a fourdimensional ellipsoidal hypersurfaces in $\mathbf{x}_{\perp}-\mathbf{x}_{\perp}^{\prime}$ phase space where $\mathcal{A}^{2}=$ const.

Characteristics of some simple choices of functions $f\left(\mathcal{A}^{2}\right)$ satisfying the normalization constraint (62) and the moment constraint (63) are given in Table II. For the $\mathrm{KV}$ case listed, the LCS distribution reduces to the KV equilibrium discussed in Sec. III A and is an exact equilibrium distribution. For any choice of $f\left(\mathcal{A}^{2}\right)$ other than the delta-function KV form, the LCS distribution is not a consistent equilibrium for finite space-charge intensity because the argument $\mathcal{A}^{2}$ is a single-particle invariant only for linear space-charge fields within the beam. General choices of $f\left(\mathcal{A}^{2}\right)$ result in density profiles $n=$ $(\lambda / q) \int d^{2} x_{\perp}^{\prime} f$ with nonuniform, elliptic-symmetry density profiles (see Table II). The nonuniform ellipticsymmetry space charge will generate nonlinear self-field forces within the beam and the $\mathcal{A}_{j}^{2}$ will evolve, changing the form of the LCS distribution. However, in the limit of vanishing space-charge intensity (i.e., $Q \rightarrow 0$ ), the LCS distributions are exact equilibria for general choices of $f\left(\mathcal{A}^{2}\right)$ satisfying Eqs. (62) and (63). In this warm-beam limit the $\mathcal{A}_{j}^{2}$ are invariants of a single particle evolving in linear applied-focusing fields. The warm-beam limit of the LCS distribution with a Gaussian choice of $f$ listed in Table II represents a standard initial particle distribution in accelerator simulations of beams with weak spacecharge intensity. Original applications of the LCS distributions appeared to be targeted for use in modeling beams with weak relative space-charge forces by including leading-order space-charge corrections by modeling space-charge forces as linear as would arise in a uniformdensity rms-equivalent beam in spite of the actual density distribution of the beam [50,51]. Discussions and tests presented here cover this original context as well as strong space-charge regimes.

Loading the LCS distribution specified by Eqs. (60)(64) in a direct Vlasov code is straightforward. The phasespace grid should be chosen to adequately resolve the distribution structure consistent with the choice of $f\left(\mathcal{A}^{2}\right)$ made and the ensuing evolution associated with the nonequilibrium form. Distribution projections listed in Table II provide a guide for characteristic resolutions needed for a range of choices in $f\left(\mathcal{A}^{2}\right)$.

An elegant procedure to load the LCS distribution using macroparticles in PIC simulations has been formulated by Batygin [50,51]. This procedure can be summarized as follows. First, canonical transformations analogous to those employed in Appendix $\mathrm{C}$ can be applied to calculate the distribution of $\mathcal{A}$ in terms of a probability transform. The resulting equation

$$
\pi^{2} \varepsilon_{x} \varepsilon_{y} \int_{0}^{\mathcal{A}^{2}} d U U f(U)=\hat{u}_{\mathcal{A}}
$$

is solved for the smallest positive real solution $\mathcal{A}\left(\hat{u}_{\mathcal{A}}\right)$ for a uniformly distributed random number $\hat{u}_{\mathcal{A}} \in[0,1)$. The transformation (67) must be solved numerically for general choices of $f$. If a large number of macroparticles are loaded, analogously to the cases discussed in Sec. III B, the transform can be presolved on a grid of values for $\hat{u}_{\mathcal{A}} \in[0,1]$ and interpolation employed for increased numerical efficiency. For the choices of functions $f$ in Table II the transformation (67) can be simplified, and in some cases analytically solved, as indicated. With $\mathcal{A}\left(\hat{u}_{\mathcal{A}}\right)$

\begin{tabular}{|c|c|c|c|c|}
\hline & \multicolumn{4}{|c|}{ Distribution name } \\
\hline & KV & Waterbag & Parabolic & Gaussian \\
\hline Definition $f\left(\mathcal{A}^{2}\right)$ & $\frac{1}{\pi^{2} \varepsilon_{x} \varepsilon_{y}} \delta\left(\mathcal{A}^{2}-1\right)$ & $\frac{8}{9 \pi^{2} \varepsilon_{x} \varepsilon_{y}} \Theta\left(1-\frac{2}{3} \mathcal{A}^{2}\right)$ & $\frac{3}{2 \pi^{2} \varepsilon_{2} \varepsilon_{y}}\left(1-\frac{1}{2} \mathcal{A}^{2}\right) \Theta\left(1-\frac{1}{2} \mathcal{A}^{2}\right)$ & $\frac{4}{\pi^{2} \varepsilon_{x} \varepsilon_{y}} e^{-2 \mathcal{A}^{2}}$ \\
\hline $\begin{array}{l}\text { Projections: } \\
\int d^{2} x_{\perp}^{\prime} f\end{array}$ & $\frac{1}{\pi r_{x} r_{y}} \Theta\left(1-\xi^{2}\right)$ & $\frac{4}{3 \pi r_{x} r_{y}}\left(1-\frac{2}{3} \xi^{2}\right) \Theta\left(1-\frac{2}{3} \xi^{2}\right)$ & $\frac{3}{2 \pi r_{x} r_{y}}\left(1-\frac{1}{2} \xi^{2}\right)^{2} \Theta\left(1-\frac{1}{2} \xi^{2}\right)$ & $\frac{2}{\pi r_{x} r_{y}} e^{-2 \xi^{2}}$ \\
\hline $\int d y \int d y^{\prime} f$ & $\frac{1}{\pi \varepsilon_{x}} \Theta\left(1-\mathcal{A}_{x}^{2}\right)$ & $\frac{4}{3 \pi \varepsilon_{x}}\left(1-\frac{2}{3} \mathcal{A}_{x}^{2}\right) \Theta\left(1-\frac{2}{3} \mathcal{A}_{x}^{2}\right)$ & $\frac{3}{2 \pi \varepsilon_{x}}\left(1-\frac{1}{2} \mathcal{A}_{x}^{2}\right)^{2} \Theta\left(1-\frac{1}{2} \mathcal{A}_{x}^{2}\right)$ & $\frac{2}{\pi \varepsilon_{x}} e^{-2 \mathcal{A}_{x}^{2}}$ \\
\hline $\int d x \int d x^{\prime} f$ & $\frac{1}{\pi \varepsilon_{y}} \Theta\left(1-\mathcal{A}_{y}^{2}\right)$ & $\frac{4}{3 \pi \varepsilon_{y}}\left(1-\frac{2}{3} \mathcal{A}_{y}^{2}\right) \Theta\left(1-\frac{2}{3} \mathcal{A}_{y}^{2}\right)$ & $\frac{3}{2 \pi \varepsilon_{y}}\left(1-\frac{1}{2} \mathcal{A}_{y}^{2}\right)^{2} \Theta\left(1-\frac{1}{2} \mathcal{A}_{y}^{2}\right)$ & $\frac{2}{\pi \varepsilon_{y}} e^{-2 \mathcal{A}_{y}^{2}}$ \\
\hline Probability & $\Theta\left(\mathcal{A}^{2}-1\right)=\hat{u}_{\mathcal{A}}$ & $\mathcal{A}^{4}=\frac{9}{4} \hat{u}_{\mathcal{A}}$ & $\mathcal{A}^{6}-3 \mathcal{A}^{4}+4 \hat{u}_{\mathcal{A}}=0$ & $\left(1+2 \mathcal{A}^{2}\right) e^{-2 \mathcal{A}^{2}}=1-\hat{u}_{\mathcal{A}}$ \\
\hline Transform & $\begin{array}{c}\Rightarrow \mathcal{A}=1 \\
\left(\text { for } \hat{u}_{\mathcal{A}}=1\right)\end{array}$ & $\Rightarrow \mathcal{A}=\sqrt{\frac{3}{2} \sqrt{\hat{u}_{\mathcal{A}}}}$ & $\begin{array}{c}\Rightarrow \mathcal{A}=\sqrt{1-2 \cos \left(\frac{\alpha-2 \pi}{3}\right)} \\
\alpha=\cos ^{-1}\left(1-2 \hat{u}_{\mathcal{A}}\right)\end{array}$ & Solve numerically \\
\hline
\end{tabular}

TABLE II. Characteristics of linear-field Courant-Snyder distributions generated for choices of $f\left(\mathcal{A}^{2}\right)$. Here, $\xi^{2} \equiv\left(x / r_{x}\right)^{2}+$ $\left(y / r_{y}\right)^{2}$. 
specified, values of $\mathcal{A}_{x} \geq 0$ and $\mathcal{A}_{y} \geq 0$ consistent with $\mathcal{A}^{2}=\mathcal{A}_{x}^{2}+\mathcal{A}_{y}^{2}$ are set by taking

$$
\mathcal{A}_{x}=\mathcal{A} \sqrt{\hat{u}_{\varphi}}, \quad \mathcal{A}_{y}=\mathcal{A} \sqrt{1-\hat{u}_{\varphi}},
$$

with $\hat{u}_{\varphi} \in[0,1)$ an independent, uniformly distributed random number. This form applies to all choices of $f$ and is taken to statistically represent the total oscillation amplitude $\mathcal{A}^{2}$ equally in the $x$ and $y$ planes [96]. Then, macroparticle phase-space coordinates are set using a phase-amplitude formulation [97] to uniformly populate oscillations in the elliptical phase spaces represented by the values of the $\mathcal{A}_{j}$ by taking

$$
\begin{array}{ll}
x=\mathcal{A}_{x} r_{x} \cos \beta_{x}, & x^{\prime}=\mathcal{A}_{x}\left(r_{x}^{\prime} \cos \beta_{x}-\frac{\varepsilon_{x}}{r_{x}} \sin \beta_{x}\right), \\
y=\mathcal{A}_{y} r_{y} \cos \beta_{y}, & y^{\prime}=\mathcal{A}_{y}\left(r_{y}^{\prime} \cos \beta_{y}-\frac{\varepsilon_{y}}{r_{y}} \sin \beta_{y}\right) .
\end{array}
$$

Here, the $\beta_{j}$ are betatron phases set as

$$
\beta_{j}=2 \pi \hat{u}_{j},
$$

with independent, uniformly distributed random numbers $\hat{u}_{j} \in[0,1)$ to uniformly distribute the oscillations in phase about the elliptical symmetry phase space.

It is worth pointing out that the Gaussian choice of $f$ indicated in Table II can be loaded using similar methods to those presented in Sec. IIIC rather than solving the probability transform in Eq. (67) (with the reduced form in Table II). Employing the factorization properties of Gaussian-distributed probability densities, it is straightforward to demonstrate that the Gaussian distribution can be alternatively loaded as

$$
\begin{array}{rlrl}
x=\frac{r_{x}}{2} \hat{g}_{x}, & y & =\frac{r_{y}}{2} \hat{g}_{y}, \\
x^{\prime}=\frac{r_{x}^{\prime}}{r_{x}} x+\frac{\varepsilon_{x}}{2 r_{x}} \hat{g}_{x^{\prime}}, & y^{\prime}=\frac{r_{y}^{\prime}}{r_{y}} y+\frac{\varepsilon_{y}}{2 r_{y}} \hat{g}_{y^{\prime}} .
\end{array}
$$

Here, $\hat{g}_{j}$ and $\hat{g}_{j^{\prime}}$ are Gaussian distributed (or truncated Gaussian for bounded phase space as discussed in Sec. III C) random numbers with unit variance.

Analogous to the cases discussed in Secs. III A, III B, and III C, the random numbers employed above to load the LCS distributions can be replaced by ordered sets of numbers to reduce initial statistical noise for a finite number of macroparticles.

Transverse slice PIC simulations illustrating the initial transient (few lattice period) evolution of the LCS distributions in a periodic FODO quadrupole transport channel are shown in Figs. 3-5. Evolutions associated with both waterbag and Gaussian choices of the function $f$ listed in Table II are shown. The simulations and parameter choices are described in Sec. II and the beam envelope is initially rms-envelope matched to the FODO lattice [80]. Simu- lations are shown for weaker $\left(\sigma_{0}=45^{\circ}\right)$ and stronger $\left(\sigma_{0}=70^{\circ}\right)$ applied-focusing strengths, each case for weak $\left(\sigma / \sigma_{0}=0.9\right)$ and strong $\left(\sigma / \sigma_{0}=0.2\right)$ relative space-charge strength. These values are selected to be parametrically removed from regions where beam transport in periodic alternating-gradient focusing channels is expected to become unstable due to the intrinsic structure of orbits near the beam edge [16,35]. Loads are generated for the waterbag $f$ case using uniformly distributed pseudorandom numbers [employing the analytic probability transform in Table II and Eqs. (68)-(70)] and Gaussiandistributed pseudorandom numbers in the Gaussian $f$ case [using Eq. (71)]. Initial distribution projections numerically calculated by binning macroparticles loaded were checked against analytically calculated projections (see Table II) to verify the validity of numerical procedures employed. Also the Gaussian distribution loading method based on Eq. (71) was carefully cross-checked against the transform method using results in Table II and Eqs. (68)(70). High macroparticle statistics are employed so the evolution of the beam density (shown every lattice period) can be observed with minimal noise. Only modest statistics are necessary for converged emittance evolutions.

The simulations clearly illustrate the expected result: that for weak relative space-charge forces $\left(\sigma / \sigma_{0} \sim 1\right)$ the LCS distributions are fairly well adapted to the transport channel and the subsequent evolutions of the distributions from the initial state are relatively small, whereas for large relative space-charge forces $\left(\sigma / \sigma_{0}\right.$ small) the lack of local force balance associated with the inconsistent use of linear-field Courant-Snyder invariants to define the distributions launches strong, transient-wave perturbations. This effect is clearly seen in the density profile evolutions in Fig. 3. In these plots, profiles of the beam density $n$ along the principal $x$ and $y$ axes are calculated from the gridded charge density in the simulation with no additional smoothing. The beam density is normalized by an rms average measure, $\lambda /\left(q \pi r_{x} r_{y}\right)$, so values not equal to unity indicate deviations from an rms-equivalent $\mathrm{KV}$ beam. Density profiles are shown at each lattice period of the evolution (in separate colors) with variations indicating deviations from periodic equilibrium conditions rather than numerical errors. Profiles along the $x$ and $y$ axesare shown separately, because the evolution introduces asymmetries between the planes. Evolutions in the rms-edge emittances $\varepsilon_{j}$ corresponding to the density evolutions in Fig. 3 are shown in Fig. 4. The emittances are calculated from Eq. (24) with the $x$ and $y$ emittances shown in black and red. Note that the $x$ - and $y$-plane evolutions in both the density profiles and emittances vary between the planes due to both the phase of the launching condition of the load within the lattice period (taken between quadrupoles) and the lack of system axisymmetry (i.e., $\partial / \partial \theta \neq 0$ ). The $x$ and $y$ emittances tend to evolve out of phase and plane average emittances [i.e., $\left(\varepsilon_{x}+\varepsilon_{y}\right) / 2$ or $\left.\sqrt{\varepsilon_{x} \varepsilon_{y}}\right)$ evolve less 
a) Waterbag: $\sigma=45^{\circ}, \sigma / \sigma=0.9$

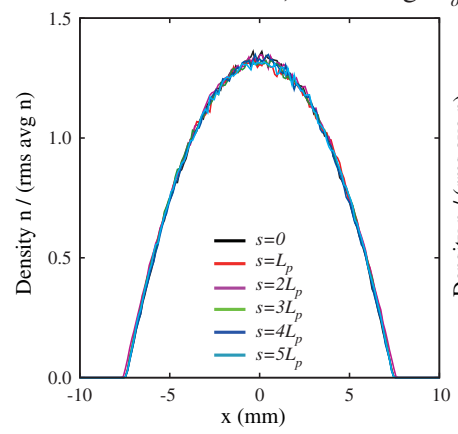

c) Waterbag: $\sigma_{o}=70^{\circ}, \sigma / \sigma_{o}=0.9$
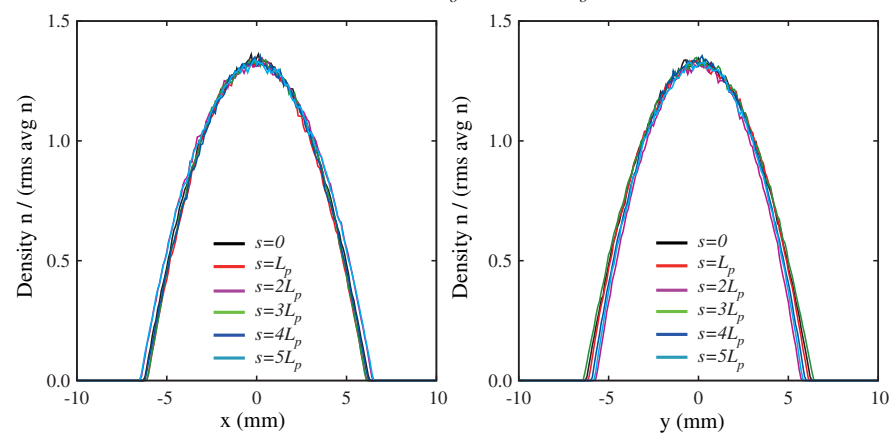

e) Gaussian: $\sigma_{o}=45^{\circ}, \sigma / \sigma_{o}=0.9$
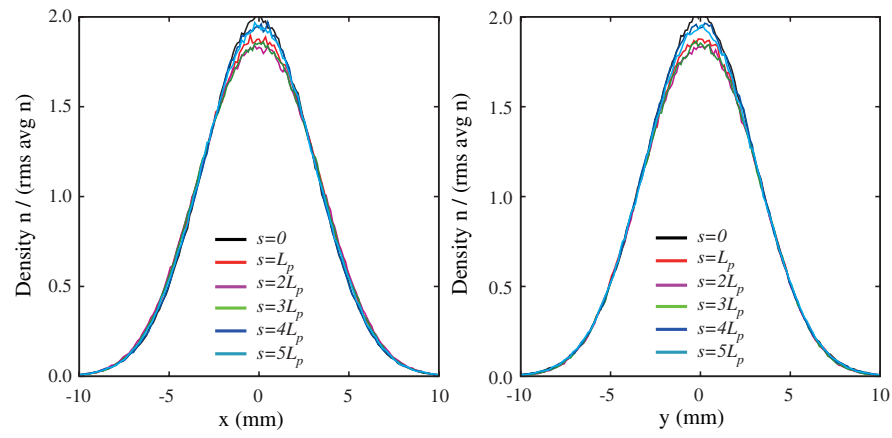

g) Gaussian: $\sigma_{o}=70^{\circ}, \sigma / \sigma_{o}=0.9$
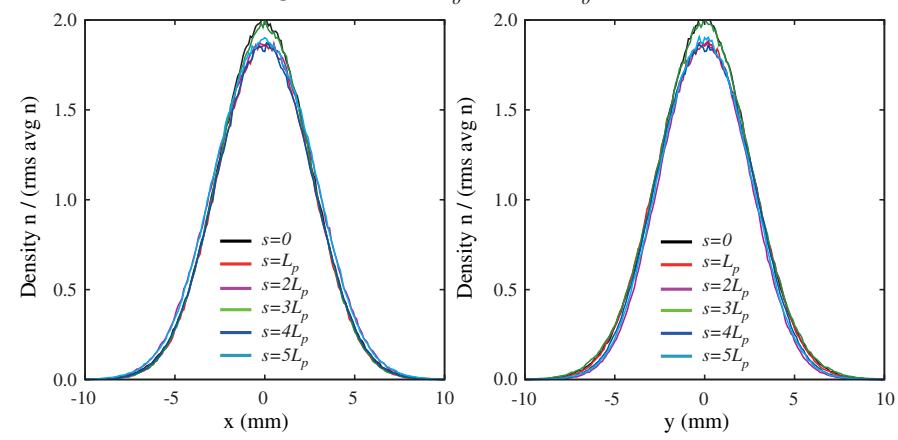

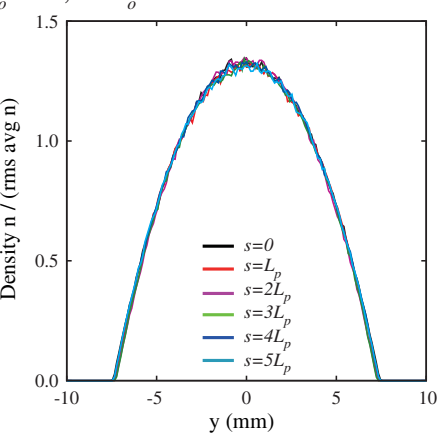

10
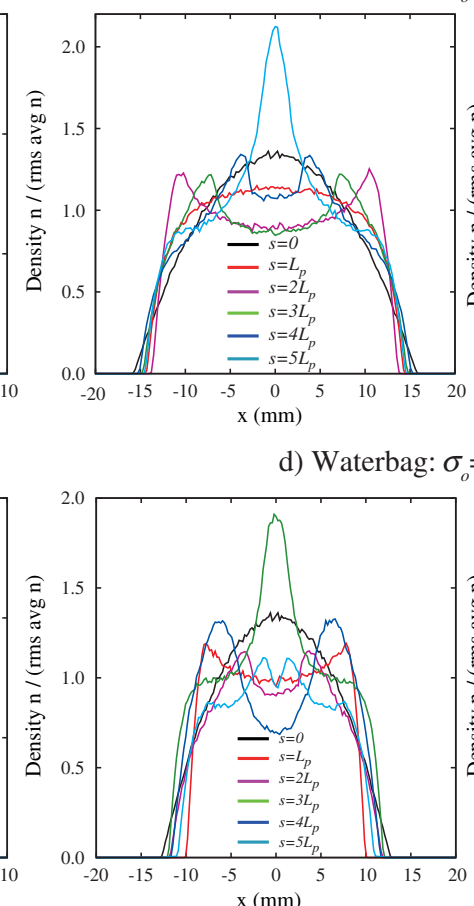

b) Waterbag: $\sigma_{o}=45^{\circ}, \sigma / \sigma_{o}=0.2$
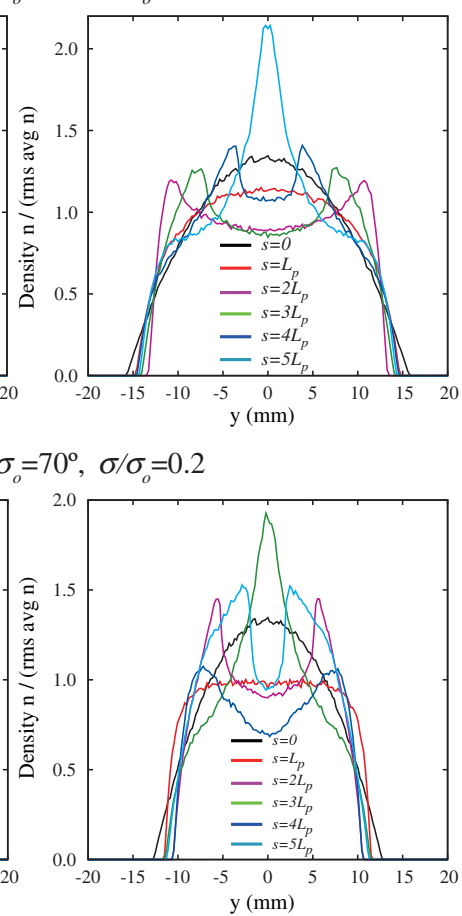

f) Gaussian: $\sigma_{o}=45^{\circ}, \sigma / \sigma_{o}=0.2$
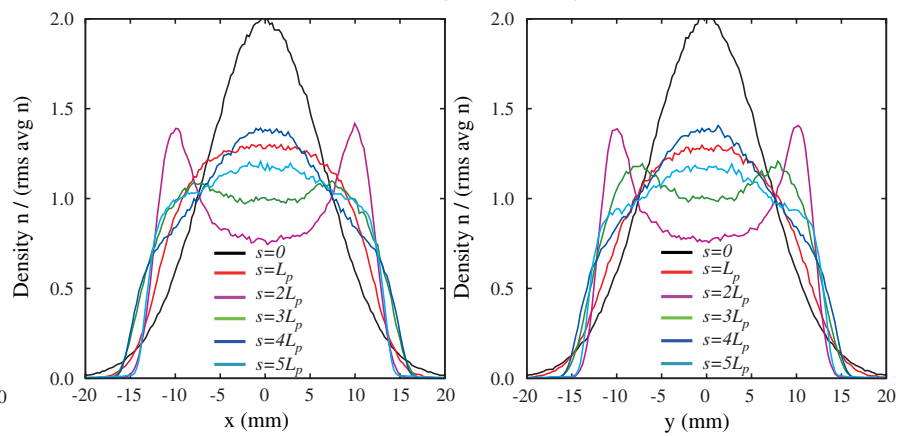

h) Gaussian: $\sigma_{o}=70^{\circ}, \sigma / \sigma_{o}=0.2$
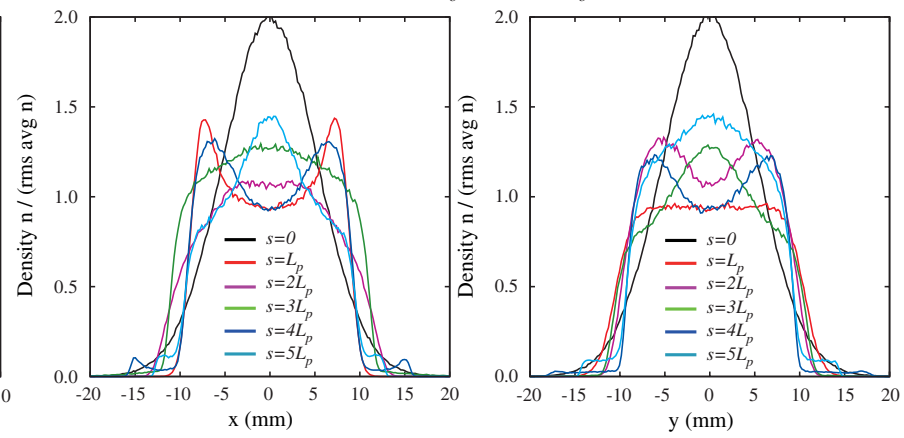

FIG. 3. (Color) Transient evolution of the beam density $n$ of initial LCS distribution loads for: (a) waterbag $f, \sigma_{0}=45^{\circ}, \sigma / \sigma_{0}=0.9$; (b) waterbag $f, \sigma_{0}=45^{\circ}, \sigma / \sigma_{0}=0.2$; (c) waterbag $f, \sigma_{0}=70^{\circ}, \sigma / \sigma_{0}=0.9$; (d) waterbag $f, \sigma_{0}=70^{\circ}, \sigma / \sigma_{0}=0.2$; (e) Gaussian $f, \sigma_{0}=45^{\circ}, \sigma / \sigma_{0}=0.9$; (f) Gaussian $f, \sigma_{0}=45^{\circ}, \sigma / \sigma_{0}=0.2$; (g) Gaussian $f, \sigma_{0}=70^{\circ}, \sigma / \sigma_{0}=0.9$; and (h) Gaussian $f$, $\sigma_{0}=70^{\circ}, \sigma / \sigma_{0}=0.2$. Density profiles are shown along the principal $x$ and $y$ axes at lattice period intervals. (B-DYN: $N_{r}=50$, $N_{\text {ppg }}=4 k, N_{s}=100, N_{p} \simeq 3$.)

but are not conserved. System (beam kinetic plus total field) energy need not be conserved because the appliedfocusing lattice can transfer energy into or out of the system. However, if rms matching is maintained, system energy is approximately conserved in an average sense over multiple lattice periods. Phase-space projections at 


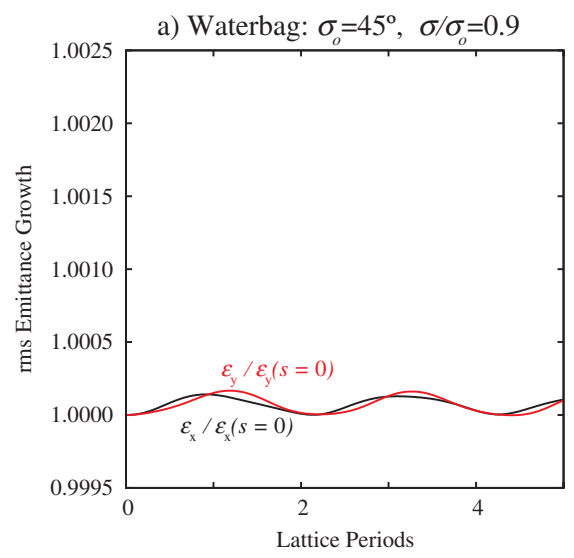

c) Waterbag: $\sigma=70^{\circ}, \sigma / \sigma=0.9$

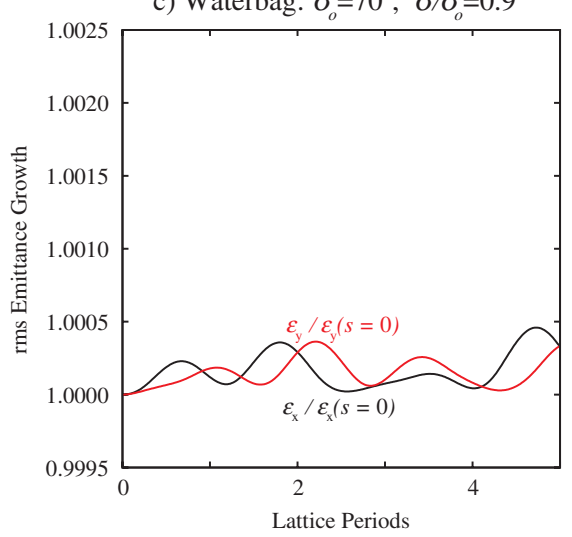

e) Gaussian: $\sigma=45^{\circ}, \sigma / \sigma=0.9$
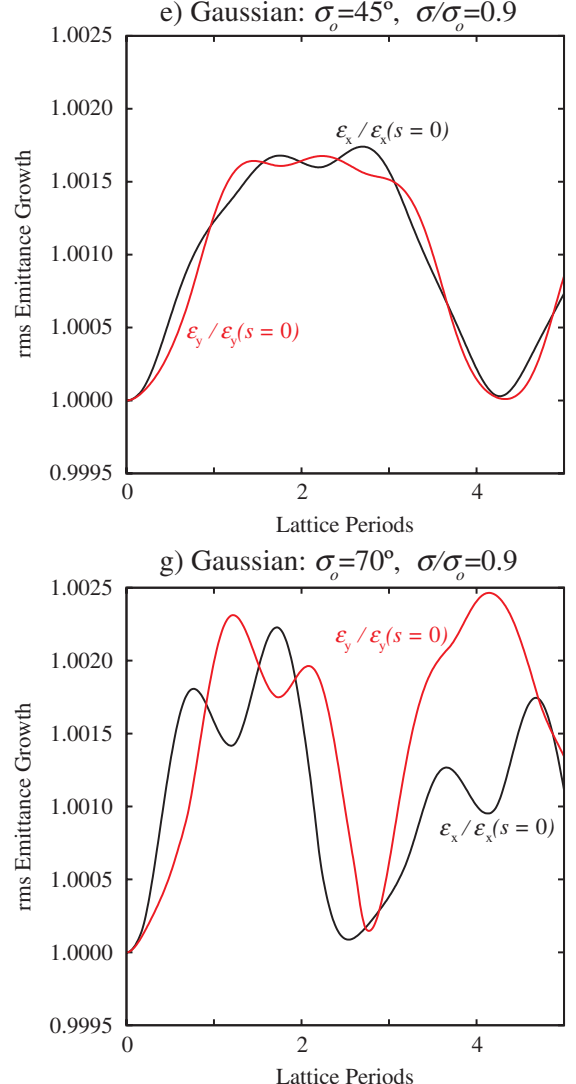

b) Waterbag: $\sigma=45^{\circ}, \sigma / \sigma=0.2$

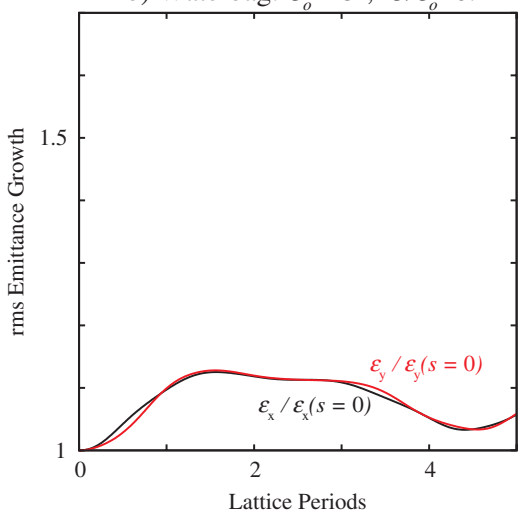

d) Waterbag: $\sigma_{o}=70^{\circ}, \sigma / \sigma_{o}=0.2$

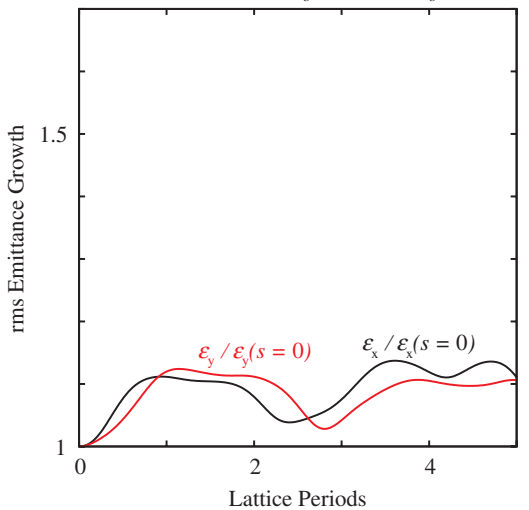

f) Gaussian: $\sigma_{o}=45^{\circ}, \sigma / \sigma=0.2$

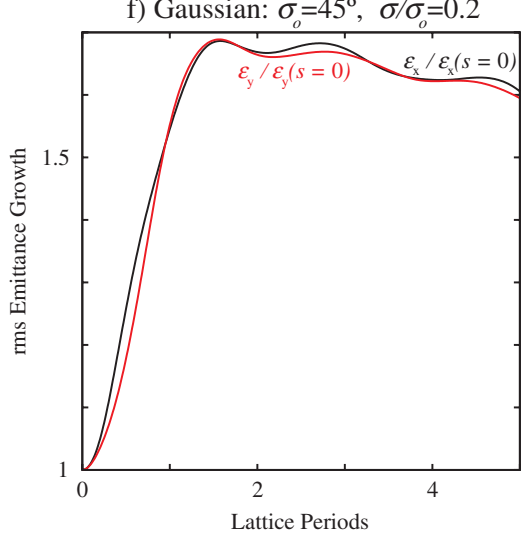

h) Gaussian: $\sigma_{o}=70^{\circ}, \sigma / \sigma_{o}=0.2$

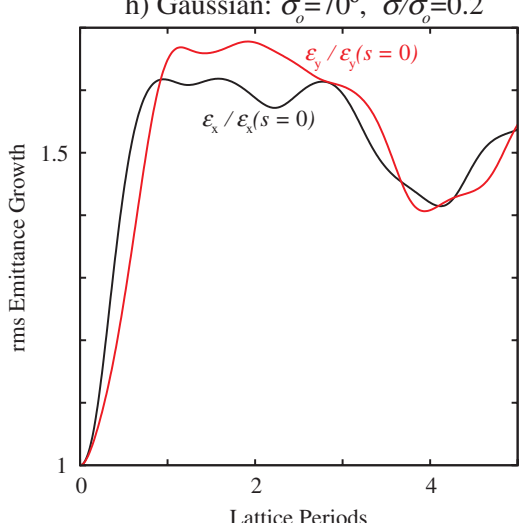

FIG. 4. (Color) Evolution of the beam rms-edge emittances $\varepsilon_{x}$ and $\varepsilon_{y}$ as a function of lattice periods $\left(s / L_{p}\right)$ for the simulations shown in Fig. 3. 

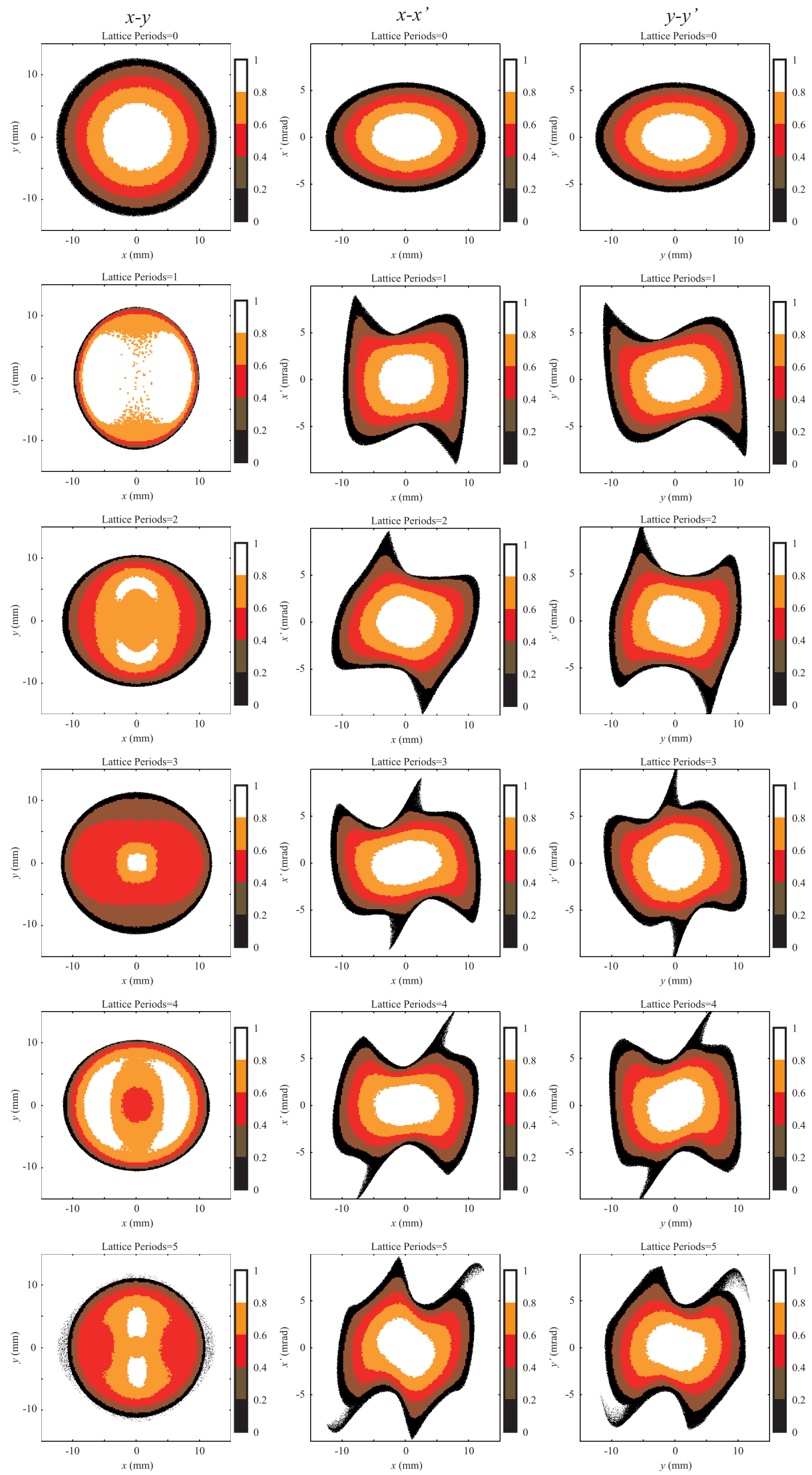

FIG. 5. (Color) Evolution of phase-space projections in $x-y, x-x^{\prime}$, and $y-y^{\prime}$ for the initial LCS waterbag distribution shown in Figs. 3 and 4 with $\sigma_{0}=70^{\circ}$ and $\sigma / \sigma_{0}=0.2$. Projections (columns) are shown at lattice period intervals (rows). 
lattice period intervals for one evolution are shown in Fig. 5. The $x-y, x-x^{\prime}$, and $y-y^{\prime}$ projections illustrate further characteristics of the large waves launched due to the lack of force balance in the beam distribution when space charge is strong. In the $x-x^{\prime}$ projections, $x^{\prime}$ represents $x^{\prime}-$ $r_{x}^{\prime}\left(x / r_{x}\right)$ with $r_{x}$ and $r_{x}^{\prime}$ calculated from Eq. (23) (i.e., in an rms-equivalent beam sense). This transformation removes the tilt angle of the ellipse associated with the coherent flow while conserving local $x-x^{\prime}$ phase-space area and thereby better illustrate distribution distortions. An analogous transformation is made in the $y-y^{\prime}$ projections. A random sampling of particles is plotted to represent the distribution. Colors of the plotted particles represent relative densities of the projections as indicated (in arbitrary units).

Despite the strength of the transient evolution for strong space charge, the rms-envelope radii $r_{j}$ of the distributions remain well matched to the focusing lattice and the emittance evolutions are relatively modest. This is not surprising given that choices of initial distributions even further out of local force balance are also observed to remain relatively well matched with modest emittance growth [15]. Waves launched by the lack of local consistency in the initial distribution tend to drive the profile to a more uniform-density beam through phase mixing, Landau damping, and nonlinear interactions. Such relaxations tend to result in increased beam emittance because the more uniform profiles have lower field energy and the energy difference between the initial and relaxed state (in an approximate sense: $x$-varying focusing forces can also transfer energy into and out of the beam during the period and details of such processes can vary especially in the initial transient evolution) is available to drive increases in incoherent spreads $[15,31,67,68]$. Longer simulations suggest that the distribution can relax to a state better adapted to the transport channel with a significant, but reduced spectrum of residual oscillations persisting. The relaxed state tends to be more nearly plane equilibrated with $\varepsilon_{x} \sim$ $\varepsilon_{y}$. Propagation distances necessary for relaxation can be significant and are difficult to determine because the relaxation distance varies with the strength of the applied focusing $\left(\sigma_{0}\right)$ and the relative space-charge strength $\left(\sigma / \sigma_{0}\right)$. Numerical approximations can also induce effective, nonphysical relaxations that are difficult to separate from other processes. This can further complicate the relaxation issue: very large simulations can be necessary for proper, physical convergence. Batygin has explored alternative techniques where nonlinear terms can be added to the applied-focusing forces so that the total applied plus space-charge force acting on the particles is linear, and then adiabatically decreasing the applied nonlinear force [63]. In any event, for strong relative space-charge forces it is desirable to generate improved, but still relatively simple loads with smooth distributions that are more equilibriumlike with lesser transient-wave evolution to simplify appli- cations and interpretation of results. This issue is addressed in Sec. IV.

\section{PSEUDOEQUILIBRIUM DISTRIBUTIONS}

We improve on the classes of specified kinetic distributions reviewed in Sec. III for Vlasov simulations of unbunched or weakly bunched beams with high space-charge intensity in nonconstant linear-focusing lattices by formulating ansatz distributions which retain desirable features of the reviewed distributions while mitigating problematic ones. Desirable features include a smooth, nonsingular distribution structure, density projections that reflect Debye screening for high space-charge intensity, and preservation of the low-order (envelope model) structure consistent with the KV distribution. Specifically, we formulate a class of "pseudoequilibrium" distributions $[67,68]$ that are straightforward to specify, and can have both smooth core structure and more nearly equilibriumlike properties that would be expected for an initial beam better adapted to a linear transport channel. The procedure (Sec. IVA) is conceptually simple to formulate through a series of transformations motivated by results in Sec. III. Beam slices are specified by rms-equivalent parameters, which are then mapped to a local rms matched continuous-focusing equilibrium distribution with self-consistent Debye screening. The distribution is then transformed back to a form more appropriate for nonconstant linear-focusing forces. Following the description of the method, example pseudoequilibrium loads are self-consistently simulated (Sec. IV B) in a periodic FODO quadrupole transport channel to illustrate results. The simulations verify improved, closer to equilibriumlike properties of the pseudoequilibrium distributions relative to the standard distributions reviewed in Sec. III.

The pseudoequilibrium procedure is formulated for a beam in an applied-focusing lattice with linear-focusing functions $\kappa_{j}(s)$ that can vary arbitrarily in $s\left(\kappa_{j} \neq\right.$ const; $j=x, y$ ). Nonlinear (or skew coupled) applied-field components can exist but are ignored in the specification of the $\kappa_{j}$ (a proper inclusion would greatly complicate the formulation since it would require the application of more complicated Courant-Snyder forms; see Ref. [98]). The beam need not be rms matched to the linear-focusing lattice and the procedure can be applied to generate transverse or full 3D beam distributions when axial variations are sufficiently slow where self-fields can be approximated as 2D transverse fields. For either the 4D or 6D phasespace cases, axial phase-space coordinates of the particles are regarded as specified (in the $s$ slice for transverse loads). The pseudoequilibrium distribution is assumed to have the form

$$
f=f_{\perp}\left(\mathbf{x}_{\perp}, \mathbf{x}_{\perp}^{\prime} ; s\right) f_{z}\left(z, p_{z}\right),
$$

where $z$ and $p_{z}$ are the longitudinal particle coordinate and momentum and $f_{z} \geq 0$ is the longitudinal distribution. 
The connection between $s$ and $z$ must be specified and the total number of particles within the beam $N=$ $\int d z \int d p_{z} \int d^{2} x_{\perp} \int d^{2} x_{\perp}^{\prime} f$ sets the normalization of $f_{z}$. A variety of longitudinal distributions $f_{z}$, such as the Neuffer distribution [99], can be applied to model the beam ends.

\section{A. Pseudoequilibrium procedure}

In the pseudoequilibrium procedure, initial transverse particle phase-space coordinates $\left(x, y, x^{\prime}, y^{\prime}\right)$ are loaded as follows.

Step 1.-For each particle at axial coordinate $s$, specify the beam perveance

$$
Q(s)=\frac{q \lambda(s)}{2 \pi \epsilon_{0} m c^{2} \gamma_{b}^{3} \beta_{b}^{2}},
$$

statistical beam edge radii

$$
r_{x}(s)=2\left\langle x^{2}\right\rangle_{\perp}^{1 / 2}, \quad r_{y}(s)=2\left\langle y^{2}\right\rangle_{\perp}^{1 / 2},
$$

envelope angles

$$
r_{x}^{\prime}(s)=\frac{2\left\langle x x^{\prime}\right\rangle_{\perp}}{\left\langle x^{2}\right\rangle_{\perp}^{1 / 2}}, \quad r_{y}^{\prime}(s)=\frac{2\left\langle y y^{\prime}\right\rangle_{\perp}}{\left\langle y^{2}\right\rangle_{\perp}^{1 / 2}},
$$

and rms-edge emittances

$$
\begin{aligned}
& \varepsilon_{x}(s)=4\left[\left\langle x^{2}\right\rangle_{\perp}\left\langle x^{\prime 2}\right\rangle_{\perp}-\left\langle x x^{\prime}\right\rangle_{\perp}^{2}\right]^{1 / 2}, \\
& \varepsilon_{y}(s)=4\left[\left\langle y^{2}\right\rangle_{\perp}\left\langle y^{\prime 2}\right\rangle_{\perp}-\left\langle y y^{\prime}\right\rangle_{\perp}^{2}\right]^{1 / 2} .
\end{aligned}
$$

For a given beam ion and slice energy, Eqs. (73)-(76) fix the beam line charge $\lambda$ and the 2nd-order moments $\left\langle x^{2}\right\rangle_{\perp}$, $\left\langle x^{\prime 2}\right\rangle_{\perp},\left\langle x x^{\prime}\right\rangle_{\perp}$ (and corresponding $y$-plane moments) in terms of $Q, r_{j}, r_{j}^{\prime}$, and $\varepsilon_{j}$. In this specification, we have not assumed that the linear focusing functions $\kappa_{j}(s)$ are periodic. If the $\kappa_{j}$ are periodic, the envelope radii $r_{j}$ need not be matched to the focusing lattice. The mean axial factors $\beta_{b}$ and $\gamma_{b}=1 / \sqrt{1-\beta_{b}^{2}}$ are set consistently with the longitudinal distribution of particles being loaded with

$$
\beta_{b}=\left\langle\frac{v_{z}}{c}\right\rangle_{v_{z}} .
$$

Here, $\langle\cdots\rangle_{v_{z}}$ denotes an average over the axial beam velocity $v_{z}$ calculated at axial slice location $s$. The paraxial approximation of small longitudinal velocity spread is assumed to apply. In cases where a long beam is accelerating and/or longitudinally compressing/expanding, a prescribed head-to-tail $s$ variation in $\beta_{b}$ is permitted over the axial length of the beam insofar as the fractional change is small.

Step 2.-Define an rms-matched, continuously focused beam for each particle with perveance $Q(s)$, statistical edge envelope radius

$$
r_{b}(s)=\sqrt{r_{x}(s) r_{y}(s)}
$$

rms-edge emittance

$$
\varepsilon_{b}(s)=\sqrt{\varepsilon_{x}(s) \varepsilon_{y}(s)},
$$

and focusing-field strength

$$
k_{\beta 0}^{2}(s)=\frac{Q(s)}{r_{b}^{2}(s)}+\frac{\varepsilon_{b}^{2}(s)}{r_{b}^{4}(s)} .
$$

The choices in Eqs. (78)-(80) are consistent with approximating the KV envelope equations (21) as an average force-balance equation with: $r_{j}=r_{b}, \kappa_{j}=k_{\beta 0}^{2}$, and $\varepsilon_{j}=$ $\varepsilon_{b}$, where we treat $r_{b}$ as slowly varying (i.e., $r_{b}^{\prime \prime}$ negligible in the envelope equation). Alternatively, the geometricmean definitions made in Eqs. (78) and (79) can be replaced with arithmetic-mean measures [e.g., $r_{b}=\left(r_{x}+\right.$ $\left.r_{y}\right) / 2$ rather than $r_{b}=\sqrt{r_{x} r_{y}}$ ] resulting in only small differences in typical applications where beams are not highly elliptical (i.e., $r_{x} \sim r_{y}$ ) and are nearly plane equilibrated (i.e., $\varepsilon_{x} \simeq \varepsilon_{y}$ ). However, the geometric-mean definitions apply more logically in the general cases since they reflect an equivalence of beam cross-sectional area and fourdimensional phase-space volume.

Step 3.-For the rms-matched, continuously focused transverse distribution defined in step 2 , specify an axisymmetric (i.e., $\partial / \partial \theta \neq 0$ ) Vlasov equilibrium distribution

$$
f_{\perp}\left(x, y, x^{\prime}, y^{\prime} ; s\right)=f_{\perp}\left[H_{\perp}(s)\right]
$$

with a particular functional form $f_{\perp}\left(H_{\perp}\right)$ (e.g., waterbag, parabolic, thermal, ...). Here,

$$
H_{\perp}(s)=\frac{1}{2} \mathbf{x}_{\perp}^{2}+\frac{1}{2} k_{\beta 0}^{2} \mathbf{x}_{\perp}^{2}+\frac{q}{m \gamma_{b}^{3} \beta_{b}^{2} c^{2}} \phi
$$

is the transverse Hamiltonian of a beam particle and parameters employed in the definition of $f_{\perp}\left(H_{\perp}\right)$ are constrained by

$$
\begin{aligned}
\lambda(s) & =q \int d^{2} x_{\perp} \int d^{2} x_{\perp}^{\prime} f_{\perp}\left(H_{\perp}\right), \\
r_{b}^{2}(s) & =\frac{4 \int d^{2} x_{\perp} \int d^{2} x_{\perp}^{\prime} x^{2} f_{\perp}\left(H_{\perp}\right)}{\int d^{2} x_{\perp} \int d^{2} x_{\perp}^{\prime} f_{\perp}\left(H_{\perp}\right)}, \\
\frac{\varepsilon_{b}^{2}(s)}{r_{b}^{2}(s)} & =\frac{4 \int d^{2} x_{\perp} \int d^{2} x_{\perp}^{\prime} x^{\prime 2} f_{\perp}\left(H_{\perp}\right)}{\int d^{2} x_{\perp} \int d^{2} x_{\perp}^{\prime} f_{\perp}\left(H_{\perp}\right)} .
\end{aligned}
$$

Generally, a function $f_{\perp}\left(H_{\perp}\right)$ satisfying the monotonicity condition $\partial f_{\perp}\left(H_{\perp}\right) / \partial H_{\perp} \leq 0$ is preferable to correspond to a stable core distribution in the continuous limit $[30,32]$. The procedure for implementing the constraints in Eq. (83) will generally be complex because $\phi$ occurring in $H_{\perp}$ must be calculated self-consistently with the transverse Poisson equation [see Eq. (33)]

$$
\frac{1}{r} \frac{\partial}{\partial r} r \frac{\partial}{\partial r} \phi=-\frac{q}{\epsilon_{0}} \int d^{2} x_{\perp}^{\prime} f_{\perp}\left(H_{\perp}\right)
$$

for each beam slice for 3D beams. Careful analysis and scaling can reduce the number of free parameters and 
numerical work necessary to calculate $\phi$ and implement the constraints associated with particular choices of equilibrium functions $f_{\perp}\left(H_{\perp}\right)$. Formulating efficient procedures is especially important in 3D applications because constraints may need to be applied to each particle independently (each particle can be in a different transverse slice of the beam). Gridded transforms can be generated (see Sec. III B) for the needed range of equilibrium parameters to reduce numerical work. For the case of transverse applications, the constraints need only be solved once rendering efficiency issues much less important. Special numerical methods can prove necessary in analyzing the constraints for strong space charge. When space charge becomes sufficiently strong, Debye screening results in the radial density profile interior to the beam becoming very flat and the beam edge sharp (see Appendices D, E, and F).

Step 4.-Load the transverse particle phase-space coordinates $x, y, x^{\prime}, y^{\prime}$ consistent with the continuous distribution calculated in step 3 (see discussions in Sec. III B).

Step 5.-Transform the axisymmetric distribution particle coordinates loaded in step 4 to local rms equivalency in the slice of the beam. This can be accomplished with a twostep procedure by first transforming the particle coordinates as

$$
x \rightarrow x=\frac{r_{x}}{r_{b}} x, \quad y \rightarrow y=\frac{r_{y}}{r_{b}} y,
$$

and then (with the first step carried out) transforming the particle angles as

$$
x^{\prime} \rightarrow x^{\prime}=\frac{\varepsilon_{x}}{r_{x}} \frac{r_{b}^{2}}{\varepsilon_{b}^{2}} x^{\prime}+\frac{r_{x}^{\prime}}{r_{x}} x, \quad y^{\prime} \rightarrow y^{\prime}=\frac{\varepsilon_{y}}{r_{y}} \frac{r_{b}^{2}}{\varepsilon_{b}^{2}} y^{\prime}+\frac{r_{y}^{\prime}}{r_{y}} y .
$$

Denoting initial/final particle coordinates with a subscript/ superscript $i / f$, this transform can be carried out in a single step as ( $y$-plane expressions analogous):

$$
x_{f}=\frac{r_{x}}{r_{b}} x_{i}, \quad x_{f}^{\prime}=\frac{\varepsilon_{x}}{\varepsilon_{b}} \frac{r_{b}}{r_{x}} x_{i}^{\prime}+\frac{r_{x}^{\prime}}{r_{b}} x_{i} .
$$

These transforms preserve linear-force Courant-Snyder invariants of the particle distribution (see Appendix B).

The transverse beam density $n=\int d^{2} x_{\perp}^{\prime} f_{\perp}$ of the initial pseudoequilibrium distribution generated by this procedure will have $n=$ const on elliptical surfaces with $\left(x / r_{x}\right)^{2}+\left(y / r_{y}\right)^{2}=$ const within the beam. However, in contrast to the KV distribution, the density profile of the pseudoequilibrium distribution will have radial structure in $\left(x / r_{x}\right)^{2}+\left(y / r_{y}\right)^{2}$ with an edge profile that reflects the choice of $f_{\perp}\left(H_{\perp}\right)$ made. For monotonic $f_{\perp}\left(H_{\perp}\right)$ and strong space charge, Debye screening will lead to a flat charge profile within the core of the beam that falls off in a few characteristic Debye lengths near the edge where $\left(x / r_{x}\right)^{2}+\left(y / r_{y}\right)^{2} \sim 1$. The specific structure of the edge (the rapidity of the falloff, whether it reduces to zero or exponentially small values, etc.), will depend on the functional form of $f_{\perp}\left(H_{\perp}\right)$ chosen. The pseudoequilibrium distribution is exact for the case of an ideal, continuousfocusing system (see Sec. III B). For $s$-varying focusing lattices the initial pseudoequilibrium distribution will not be an exact equilibrium [except when $f_{\perp}\left(H_{\perp}\right)$ is chosen to correspond to a continuous-focusing $\mathrm{KV}$ distribution] and some initial transient evolution is expected. For stronger focusing (larger $\sigma_{0 j}$ ), this transient is expected to become more pronounced because stronger focusing will generally be more poorly approximated by the continuous limit. For sufficiently large $\sigma_{0 j}$ the beam is expected to become destabilized for any choice of $f_{\perp}\left(H_{\perp}\right)$ [16]. It should again be stressed that, although the underlying continuous distribution $f_{\perp}\left(H_{\perp}\right)$ used in the construction of the pseudoequilibrium distribution are rms matched, the resulting pseudoequilibrium distribution applies to arbitrary $s$-varying focusing lattices. If the lattice focusing functions are periodic, the initial pseudoequilibrium distribution can be envelope matched or envelope mismatched, depending on the choice of $r_{j}$ made.

\section{B. Illustrative simulations}

The pseudoequilibrium loading procedure outlined in Sec. IVA is implemented in the WARP and B-DYN PIC codes for underlying continuous waterbag, and thermal equilibrium distributions. Example transverse slice PIC simulations illustrating the initial transient evolution of the pseudoequilibrium distributions in a periodic FODO quadrupole transport channel are presented in Figs. 6-9 for distributions with underlying waterbag and thermal equilibrium form. Codes and parameter choices are described in Sec. II, and data are presented using analogous procedures and formats as presented in Secs. III B and IIID. Exceptions to this correspondence are explicitly noted. Simulations are shown for weaker $\left(\sigma_{0}=45^{\circ}\right)$ and stronger $\left(\sigma_{0}=70^{\circ}\right)$ applied-focusing strengths, each for weak $\left(\sigma / \sigma_{0}=0.9\right)$ and strong $\left(\sigma / \sigma_{0}=0.2\right)$ relative spacecharge strength. These values are selected to be parametrically removed from regions where alternating-gradient transport is expected to become unstable resulting from the intrinsic structure of orbits near the beam edge in periodic systems with strong space charge [16,35]. Beams are initially rms-envelope matched to the transport channel [80]. In all simulations digit-reversed numbers are used to generate macroparticle loads with lower statistical noise to allow better visualization of collective wave evolution. Also, large numbers of macroparticles are simulated to allow clear visualization of density profile evolutions with limited noise-only relatively modest numbers of macroparticles are necessary for converged emittance evolutions. The radial probability transform (see discussion in Sec. III B) needed to load the macroparticle coordinates are solved on a uniform mesh of 500 points with a cutoff set at the beam edge in the waterbag case and where the density 

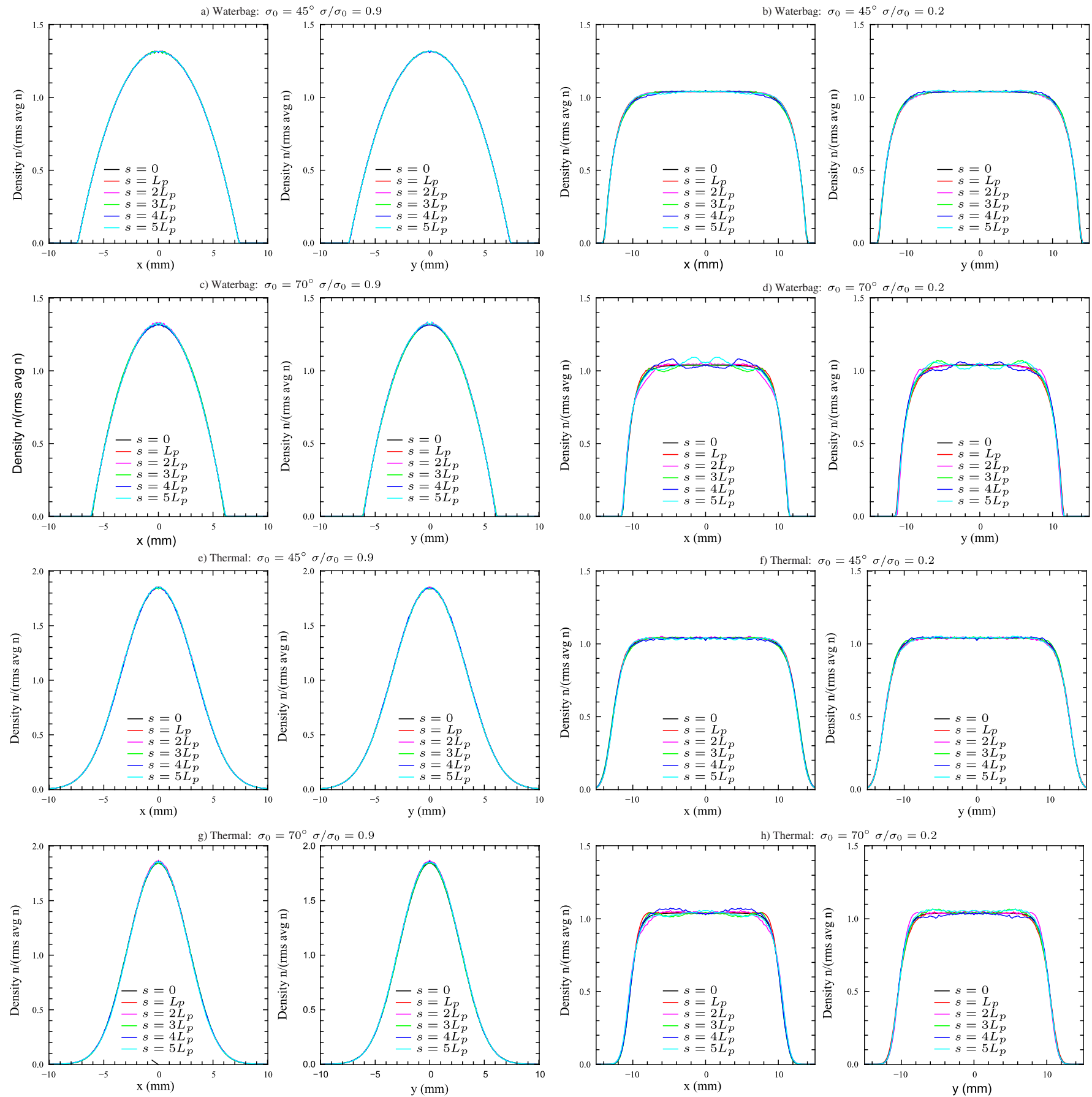

FIG. 6. (Color) Transient evolution of the beam density $n$ of initial pseudoequilibrium distribution loads for: (a) waterbag form: $\sigma_{0}=45^{\circ}, \sigma / \sigma_{0}=0.9$; (b) waterbag form: $\sigma_{0}=45^{\circ}, \sigma / \sigma_{0}=0.2$; (c) waterbag form: $\sigma_{0}=70^{\circ}, \sigma / \sigma_{0}=0.9$; (d) waterbag form: $\sigma_{0}=70^{\circ}, \sigma / \sigma_{0}=0.2$; (e) thermal form: $\sigma_{0}=45^{\circ}, \sigma / \sigma_{0}=0.9$; (f) thermal form: $\sigma_{0}=45^{\circ}, \sigma / \sigma_{0}=0.2$; (g) thermal form: $\sigma_{0}=$ $70^{\circ}, \sigma / \sigma_{0}=0.9$; (h) thermal form: $\sigma_{0}=70^{\circ}, \sigma / \sigma_{0}=0.2$. Density profiles are shown along the principal $x$ and $y$ axes at lattice period intervals. (WARP: $N_{r}=50, N_{\text {ppg }}=40 \mathrm{k}, N_{s}=100, N_{p} \simeq 3$.)

becomes exponentially small in the thermal case. Short (few-lattice-period) evolutions are simulated to display initial transient evolutions characteristic of nonequilibrium behavior. We generally find that longer evolutions have negligible emittance growth with particles remaining well confined in the beam core without large changes in distri- bution structure if parameters are chosen sufficiently far from regions where the system is expected to be unstable [16,35].

Comparing the results in Figs. 6-9 for the pseudoequilibrium distributions to the results presented in Sec. III D for analogous linear-field Courant-Snyder invariant distribu- 
a) Waterbag: $\sigma_{0}=45^{\circ}, \sigma / \sigma_{0}=0.9$
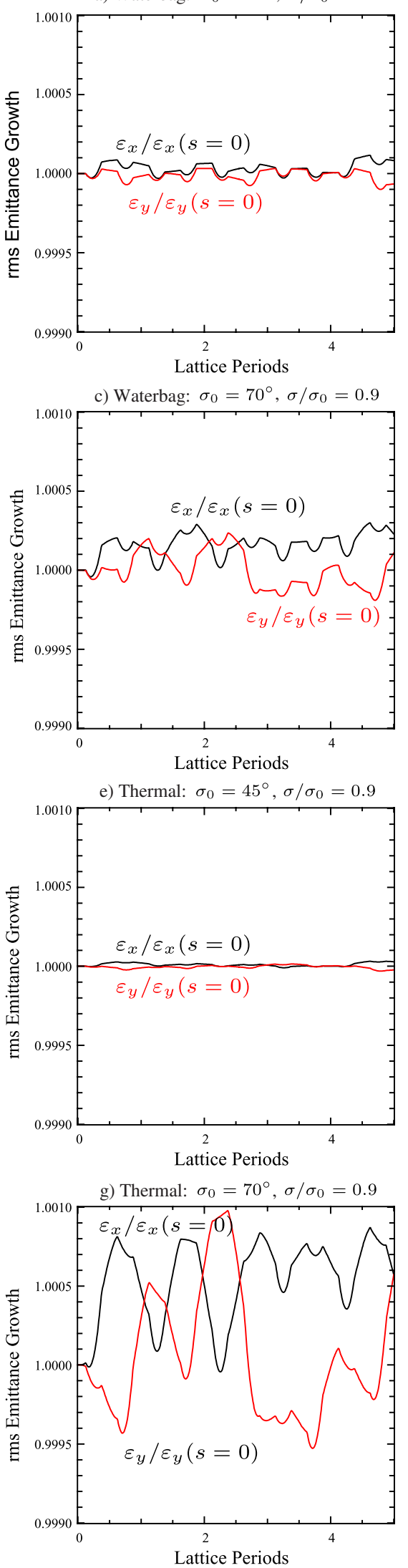

b) Waterbag: $\sigma_{0}=45^{\circ}, \sigma / \sigma_{0}=0.2$
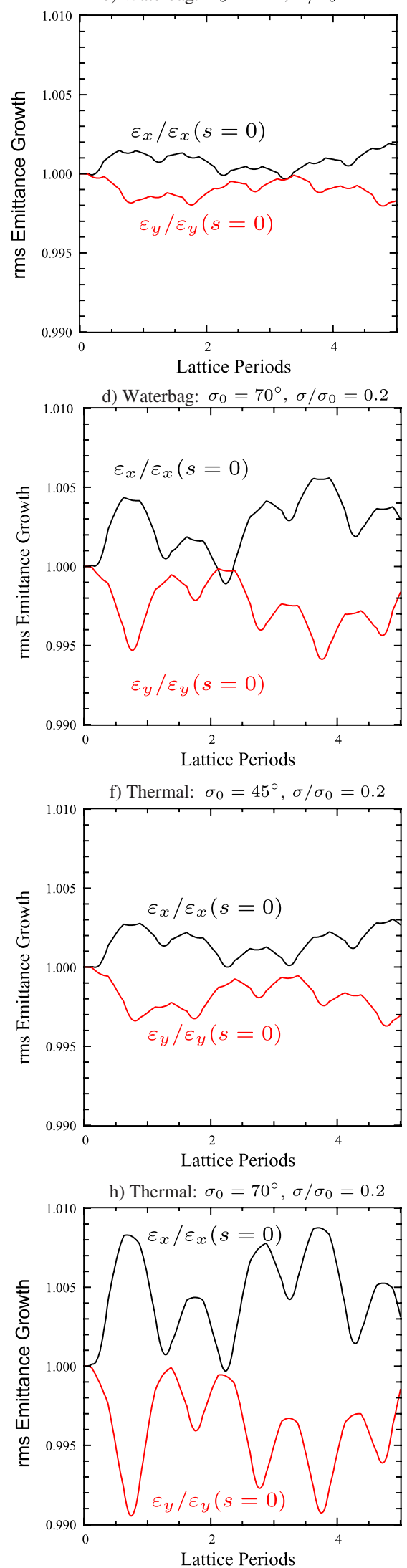

FIG. 7. (Color) Evolution of the beam rms-edge emittances $\varepsilon_{x}$ and $\varepsilon_{y}$ as a function of lattice periods $\left(s / L_{p}\right)$ for the simulations shown in Fig. 6. 

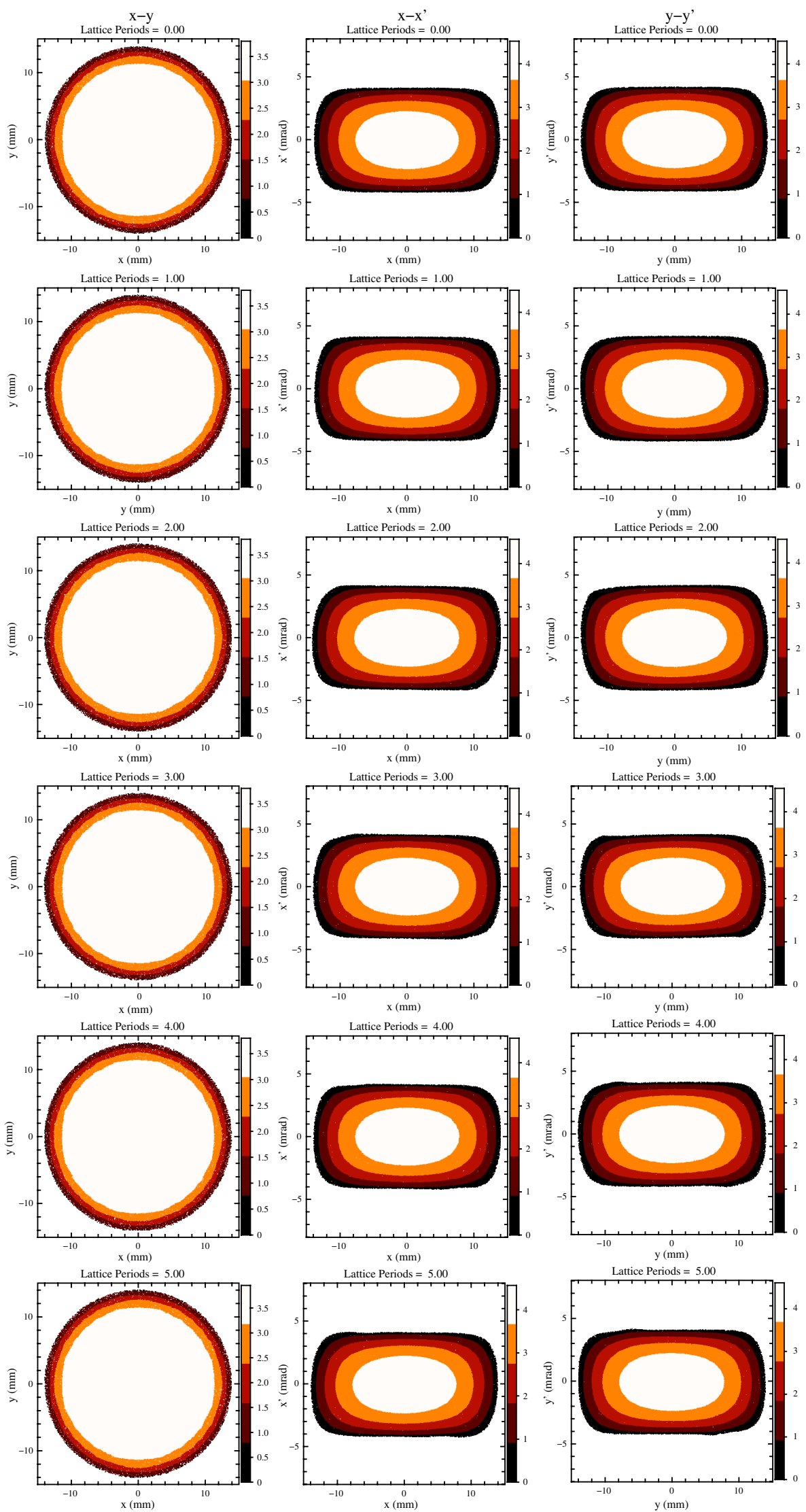

FIG. 8. (Color) Evolution of phase-space projections in $x-y, x-x^{\prime}$, and $y-y^{\prime}$ for the initial pseudoequilibrium waterbag distribution simulation shown in Figs. 6 and 7 with $\sigma_{0}=45^{\circ}$ and $\sigma / \sigma_{0}=0.2$. Projections (columns) are shown at lattice period intervals (rows). 

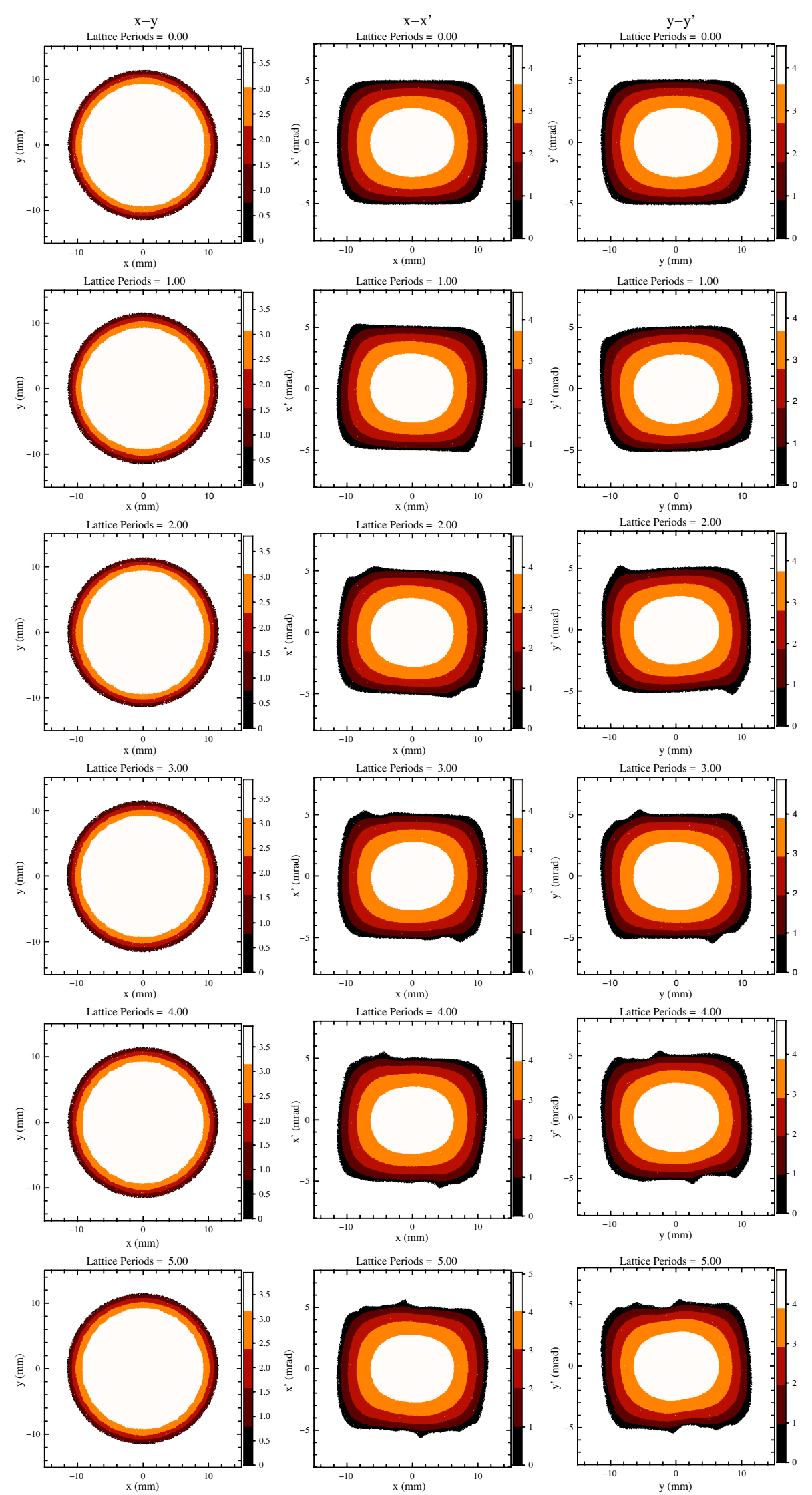

FIG. 9. (Color) Evolution in phase-space projections in $x-y, x-x^{\prime}$, and $y-y^{\prime}$ for the initial pseudoequilibrium waterbag distribution simulation shown in Figs. 6 and 7 with $\sigma_{0}=70^{\circ}$ and $\sigma / \sigma_{0}=0.2$. Projections (columns) are shown at lattice period intervals (rows). 
tions, it is clear that the pseudoequilibrium distributions are much better adapted to the applied-focusing channelparticularly for stronger relative space-charge intensity. Although collective wave perturbations are still launched from the initial pseudoequilibrium distributions, the strength of residual waves launched from the lack of detailed equilibrium form is significantly reduced indicating an initial beam that is better adapted to the transport channel. This improved adaptation is particularly apparent for cases of high space-charge intensity when contrasting the density profile evolutions in Figs. 3 and 6. Simulations of initial pseudoequilibrium distributions with weaker applied focusing (i.e., smaller $\sigma_{0}$ ) and weaker relative spacecharge intensity (i.e., higher $\sigma / \sigma_{0}$ ) have evolutions closer to equilibrium form with the distribution projections nearly periodically repeating with each lattice period. Evolutions in the rms-edge emittances $\varepsilon_{j}$ are relatively small in all cases of the pseudoequilibrium distributions because the evolutions in the charge-density profile are modest. Simulation cases in Figs. 6 and 7 that are not shown in the phase-space projections in Figs. 8 and 9 have smaller deviations. In these projection plots a numerical method is employed which shows almost all macroparticles in the low-density regions and a sampling of macroparticles in the high-density regions. This enhances visualization of perturbations near the edge of the beam relative to the sampling method employed in the corresponding phasespace projection plots in Sec. III D. Phase-space projections of the waterbag form pseudoequilibrium distribution are shown rather than projections of the thermal form pseudoequilibrium distribution because the sharp phasespace boundary of the waterbag distribution (along with plotting essentially all macroparticles near the edge) makes it easier to visualize waves associated with the lack of equilibrium form which manifest most strongly near the beam edge. In all cases, waves are launched due to lack of exact force balance near the radial edge of the beam distribution as the beam evolves in the alternating-gradient focusing structure. Note in Figs. 8 and 9 that wave perturbations are primarily launched near the edge of the distribution and only weakly perturb the core while distorting the low-density region near the beam edge most. Emittance evolutions shown in Fig. 7 are small in all cases examined-as should be expected because charge redistributions are modest and the beam envelope remains rmsenvelope matched to high accuracy $[15,31]$.

Although the pseudoequilibrium distributions are not exact equilibria, the underlying smooth continuousfocusing distributions reflect self-consistent space-charge screening and stable functional forms in the continuous limit that are expected to have less free energy relative to the KV distribution to drive wavelike instabilities. In terms of waves launched from the lack of detailed equilibrium form, the pseudoequilibrium distributions also exhibit improved performance relative to other nonequilibrium ansatz-type initial distributions such as the semiGaussian (see Sec. IIIC) and linear-field Courant-Snyder invariant (see Sec. III D) distributions that are commonly in use. These properties render the pseudoequilibrium distributions useful in probing intrinsic space-charge-related transport limits of periodic-focusing channels. Parametric simulation studies carried out with the pseudoequilibrium loads have already been applied as part of a study to better understand space-charge related transport limits in quadrupole focusing channels [16]. Finally, it should be stressed that the pseudoequilibrium distribution loads are not only applicable to periodic alternating-gradient focusing channels. The procedure applies to any lattice with $s$-varying or constant applied-focusing forces described by the focusing functions $\kappa_{j}(s)$. A simple periodic FODO lattice is employed here only for simplicity of illustration and for demanding test cases. One might expect the procedure for constructing the pseudoequilibrium distributions to work even better in the sense of approximating equilibrium properties because particle orbits in high-occupancy solenoidal transport systems are generally better approximated by particle orbits in the continuous-focusing model relative to orbits in strong (quadrupole) focusing systems. The pseudoequilibrium distributions can also be applied to simulate beam transition and matching sections, or other aperiodic transport lattices. As with the case of periodic systems, better performance can be expected for aperiodic lattices with the pseudoequilibrium distributions relative to other conventional choices of ansatz distributions when space-charge intensity is high.

\section{CONCLUSIONS}

Standard classes of distributions commonly in use for initializing transverse Vlasov simulations of charged particle beams with intense space-charge were reviewed in this paper, including the following: the KV equilibrium distribution; continuous-focusing equilibria, with detailed examples for "waterbag," "parabolic," and "thermal" forms; the nonequilibrium semi-Gaussian distribution; and nonequilibrium distributions of linear-field CourantSnyder invariants. All distributions were presented within a common notation and prescriptions were given to generate macroparticle distributions for loading PIC simulations. Care was taken to formulate the presentation in terms of standard accelerator variables (perveances, rms emittances, etc.) rather than special theoretical parameters not in common use, to render methods directly applicable to standard accelerator problems. Procedures were developed to specify loads over the full range of space-charge strength-even for continuous-focusing equilibria where high space-charge intensity can present practical difficulties. Deficiencies of the various distributions used in modeling linear-focusing channels with noncontinuous focusing forces were discussed and illustrative Vlasov 
PIC simulations were presented for initial distributions not already detailed in the literature.

Following this review, a new class of pseudoequilibrium distribution functions was derived, building on the standard classes of distributions reviewed. The pseudoequilibrium distributions were formulated to satisfy the need for a more equilibriumlike, yet simple, smooth distribution to apply in simulations of intense beams in focusing channels with linear applied forces that vary arbitrarily (other than excluding skew couplings) in the axial coordinate $s$. The pseudoequilibrium distributions are not exact equilibria of a linear-focusing channel with nonconstant appliedfocusing forces, but they are relatively simple to formulate, and have appealing physical properties expected for a relaxed beam evolving in a linear-focusing channel with space-charge driven Debye screening. The cores of the pseudoequilibrium distributions are specified by any, stable continuous-focusing equilibrium beam. Transformations that preserve linear-field Courant-Snyder invariants are then applied to map these continuous distributions to a form more appropriate for focusing channels with $s$-varying applied-focusing forces. Details are presented to generate pseudoequilibrium distributions with underlying waterbag, parabolic, and thermal equilibrium continuous-focusing forms-which cover a wide range of phase-space structure. Illustrative Vlasov PIC simulations were carried out to evolve transverse pseudoequilibrium loads in a periodic FODO quadrupole focusing channel to explicitly demonstrate the advantages of the smooth core structure in terms of diminished transient waves relative to more standard initial distributions. This more quiescent behavior can aid understanding of detailed transport physics. The pseudoequilibrium procedure can be applied to load both 2D transverse slices (4D phase space) as well as full $6 \mathrm{D}$ phase space (with specified longitudinal structure) distributions. Also, relaxation methods can be applied to initial pseudoequilibrium loads to further improve the adaptation of the beam in the sense of being more equilibriumlike in cases where the distributions should be stable. Considerable opportunities still exist for future research in equilibriumlike loads in linear-focusing channels with nonconstant focusing forces - both in terms of the intrinsic existence or nonexistence of smooth Vlasov equilibrium distribution functions and in construction of better approximate loads through improved physical insight or systematic perturbation theory.

\section{ACKNOWLEDGMENTS}

The authors wish to thank the following: D. P. Grote and J.-L. Vay for assistance with the WARP simulations and helpful discussions; J.J. Barnard, Y.K. Batygin, A. Friedman, and E. A. Startsev for helpful discussions; and S. Kawata for guidance and helpful discussions. This research was performed under the auspices of the U.S. Department of Energy at the Lawrence Livermore
National Laboratory under Contract No. W-7405-Eng-48, at Princeton Plasma Physics Laboratory under Contract No. DE-ACOZ-76-CHO-3073, and by JSPS (Japan Society for the Promotion of Science) and MEXT (Ministry of Education, Culture, Sports, Science, and Technology). Part of the research was carried out during visits by S. M. Lund to Utsunomiya University and reciprocal visits by T. Kikuchi to Lawrence Berkeley National Laboratory. These visits were part of an ongoing series of Japan-U.S. exchanges on ion-beam driven heavy ion fusion and high energy density physics.

\section{APPENDIX A: ACCELERATION EFFECTS}

In the absence of axial beam acceleration, $\gamma_{b} \beta_{b}=$ const and the particle equation of motion in the $x$ direction that is produced by the Hamiltonian (8) is

$$
x^{\prime \prime}+\kappa_{x} x=-\frac{q}{m \gamma_{b}^{3} \beta_{b}^{2} c^{2}} \frac{\partial \phi}{\partial x} .
$$

If $\gamma_{b} \beta_{b}$ is allowed to vary slowly in $s$ consistent with axial acceleration forces acting on the beam, then the equation of motion (A1) is modified as $[31,67,68]$

$$
x^{\prime \prime}+\frac{\left(\gamma_{b} \beta_{b}\right)^{\prime}}{\left(\gamma_{b} \beta_{b}\right)} x^{\prime}+\kappa_{x} x=-\frac{q}{m \gamma_{b}^{3} \beta_{b}^{2} c^{2}} \frac{\partial \phi}{\partial x} .
$$

For $\left(\gamma_{b} \beta_{b}\right)^{\prime}>0$, one may deduce from Eq. (A2) and analysis of damped harmonic oscillators [100] that the acceleration will tend to damp particle oscillations. Analogous equations hold in the $y$ plane both here and in subsequent equations.

A transformation to tilde variables is defined by taking $[67,68,101]$

$$
\tilde{x}=\sqrt{\gamma_{b} \beta_{b}} x .
$$

Then

$$
\tilde{x}^{\prime}=\sqrt{\gamma_{b} \beta_{b}} x^{\prime}+\frac{1}{2} \frac{\left(\gamma_{b} \beta_{b}\right)^{\prime}}{\sqrt{\gamma_{b} \beta_{b}}} x,
$$

and the particle $x$-x $x^{\prime}$ phase-space coordinates are related to the $\tilde{x}-\tilde{x}^{\prime}$ coordinates by

$$
x=\frac{1}{\sqrt{\gamma_{b} \beta_{b}}} \tilde{x}, \quad x^{\prime}=\frac{1}{\sqrt{\gamma_{b} \beta_{b}}} \tilde{x}^{\prime}-\frac{1}{2} \frac{\left(\gamma_{b} \beta_{b}\right)^{\prime}}{\left(\gamma_{b} \beta_{b}\right)^{3 / 2}} \tilde{x} .
$$

Some straightforward manipulation then shows that the equation of motion (A2) can be expressed as

$$
\begin{aligned}
\tilde{x}^{\prime \prime} & +\left[\kappa_{x}+\frac{1}{4} \frac{\left(\gamma_{b} \beta_{b}\right)^{\prime 2}}{\left(\gamma_{b} \beta_{b}\right)^{2}}-\frac{1}{2} \frac{\left(\gamma_{b} \beta_{b}\right)^{\prime \prime}}{\left(\gamma_{b} \beta_{b}\right)}\right] \tilde{x} \\
& =-\frac{q}{m \gamma_{b}^{2} \beta_{b} c^{2}} \frac{\partial \phi}{\partial \tilde{x}} .
\end{aligned}
$$

A transformed potential $\tilde{\phi}$ is defined as 


$$
\tilde{\phi}=\gamma_{b} \beta_{b} \phi
$$

Then the equation of motion (A6) becomes

$$
\tilde{x}^{\prime \prime}+\tilde{\kappa}_{x} \tilde{x}=-\frac{q}{m \gamma_{b}^{3} \beta_{b}^{2} c^{2}} \frac{\partial \tilde{\phi}}{\partial \tilde{x}},
$$

where

$$
\tilde{\kappa}_{x} \equiv \kappa_{x}+\frac{1}{4} \frac{\left(\gamma_{b} \beta_{b}\right)^{\prime 2}}{\left(\gamma_{b} \beta_{b}\right)^{2}}-\frac{1}{2} \frac{\left(\gamma_{b} \beta_{b}\right)^{\prime \prime}}{\left(\gamma_{b} \beta_{b}\right)}
$$

is a linear-focusing function that incorporates acceleration effects.

The equivalence of form between the equations of motion (A2) and (A8) shows that the formulation with $\gamma_{b} \beta_{b}=$ const can be applied to accelerating beams if the particle phase-space coordinates are interpreted consistently with the transformations in Eqs. (A3) and (A4). Note that in a periodic applied-focusing lattice, if the beam is to remain envelope matched in the usual sense, Eq. (A9) shows that the focusing functions $\kappa_{j}(j=x, y)$ must, in general, be adjusted such that the $\tilde{\kappa}_{j}$ maintain proper periodicity with $\tilde{\kappa}_{j}\left(s+L_{p}\right)=\tilde{\kappa}_{j}(s)$. For a lattice with discrete acceleration gaps and separated function magnets for beam focusing, this can be done approximately when the fractional gain in particle energy through each acceleration gap is small.

The transformation defined by Eqs. (A3) and (A4) is straightforward to interpret. The Jacobian of the transformation shows that phase-space area elements are related as

$$
d \tilde{x} \otimes d \tilde{x}^{\prime}=\gamma_{b} \beta_{b} d x \otimes d x^{\prime} .
$$

The $\gamma_{b} \beta_{b}$ factor compensates for acceleration induced damping in $x-x^{\prime}$ phase space. If the transformed equations of motion are linear, then single-particle Courant-Snyder invariants exist in $\tilde{x}-\tilde{x}^{\prime}$ phase space (see Appendix B), but the phase-space area associated with the Courant-Snyder invariant will be damped by the factor $1 /\left(\gamma_{b} \beta_{b}\right)$ in $x$ - $x^{\prime}$ phase space. This is the reason for the conventional application of normalized emittances that incorporate the $\gamma_{b} \beta_{b}$ factor when measuring the phase-space area in accelerating beams $[31,67,68,102]$.

Finally, it should be pointed out that if a transformed distribution $\tilde{f}_{\perp}$ is defined such that

$$
\tilde{f}_{\perp} d^{2} \tilde{x}_{\perp} d^{2} \tilde{x}_{\perp}^{\prime}=f_{\perp} d^{2} x_{\perp} d^{2} x_{\perp}^{\prime},
$$

then the Jacobian of the transformation (A5) $d^{2} \tilde{x}_{\perp} d^{2} \tilde{x}_{\perp}^{\prime}=$ $\left(\gamma_{b} \beta_{b}\right)^{2} d^{2} x_{\perp} d^{2} x_{\perp}^{\prime}$, and consequently $\tilde{f}_{\perp}$ is simply related to the beam distribution $f_{\perp}$ by

$$
\tilde{f}_{\perp}=\frac{1}{\left(\gamma_{b} \beta_{b}\right)^{2}} f_{\perp} .
$$

If one naturally defines a charge density for the transformed distribution as

$$
\tilde{\rho}=q \int d^{2} \tilde{x}_{\perp}^{\prime} \tilde{f}_{\perp}
$$

then the regular charge distribution $\rho=q \int d^{2} x_{\perp}^{\prime} f_{\perp}$ is related to $\tilde{\rho}$ by

$$
\tilde{\rho}=\frac{1}{\gamma_{b} \beta_{b}} \rho,
$$

and the transformation of the Poisson equation (9) is

$$
\left(\frac{\partial^{2}}{\partial \tilde{x}^{2}}+\frac{\partial^{2}}{\partial \tilde{y}^{2}}\right) \tilde{\phi}=-\frac{\rho}{\epsilon_{0}}=-\frac{\rho}{\gamma_{b} \beta_{b} \epsilon_{0}} .
$$

The additional factor of $1 /\left(\gamma_{b} \beta_{b}\right)$ is an expression of the weakening of transverse space-charge effects with acceleration and must be treated with care to establish the proper correspondences.

\section{APPENDIX B: LINEAR-FORCE COURANT- SNYDER INVARIANTS}

An $x$-plane particle orbit within a KV beam is described by the Hill's equation $[30-32,36,67,68]$

$$
x^{\prime \prime}(s)+\kappa_{x}(s) x(s)-\frac{2 Q x(s)}{\left[r_{x}(s)+r_{y}(s)\right] r_{x}(s)}=0 .
$$

For $Q=0$ this equation also describes an $x$-plane orbit of a single-particle moving in the linear applied-focusing fields of a lattice. In a phase-amplitude resolution of the particle orbit we take

$$
x=A_{x} \cos \psi_{x},
$$

where $A_{x}$ and $\psi_{x}$ denote $s$-varying amplitude and phase functions. Without loss of generality, $A_{x}$ and $\psi_{x}$ can be taken to satisfy $2 A_{x}^{\prime} \psi_{x}^{\prime}+A_{x} \psi_{x}^{\prime \prime}=0$, or equivalently,

$$
\psi_{x}^{\prime}=\frac{\text { const }}{A_{x}^{2}} .
$$

The amplitude is set to be $A_{x}=\mathcal{A}_{x} r_{x}$, where $\mathcal{A}_{x}$ is a positive dimensionless constant. We can then take const $=$ $\mathcal{A}_{x}^{2} \varepsilon_{x}$ in Eq. (B3) without restrictions on the generality of the solution. Then the equation of motion (B1) becomes

$$
\begin{gathered}
\mathcal{A}_{x}\left[r_{x}^{\prime \prime}+\kappa_{x} r_{x}-\frac{2 Q}{r_{x}+r_{y}}-\frac{\varepsilon_{x}^{2}}{r_{x}^{3}}\right]=0, \\
\psi_{x}^{\prime}=\frac{\varepsilon_{x}}{r_{x}^{2}} .
\end{gathered}
$$

The amplitude equation is satisfied for $\mathcal{A}_{x} \neq 0$ if the $r_{j}$ $(j=x, y)$ satisfy the KV envelope equation (21). Note that the rate of phase advance, $\psi_{x}^{\prime}=\varepsilon_{x} / r_{x}^{2}$, is independent of relative particle oscillation amplitude $\mathcal{A}_{x}$. This phaseadvance equation can be integrated to express the total phase advance $\sigma_{x}$ through an axial distance from $s=s_{i}$ to $s=s_{i}+L_{p}$ (lattice period) as 


$$
\sigma_{x}=\psi_{x}\left(s_{i}+L_{p}\right)-\psi_{x}\left(s_{i}\right)=\varepsilon_{x} \int_{s_{i}}^{s_{i}+L_{p}} \frac{d s}{r_{x}^{2}} .
$$

If the focusing lattice is periodic with period $L_{p}$, and the envelope $r_{j}$ is matched, then the "depressed" phase advance $\sigma_{x}$ [see Eq. (25)] is independent of $s_{i}$. Using $A_{x}=$ $\mathcal{A}_{x} r_{x}$ and $\psi_{x}^{\prime}=\varepsilon_{x} / r_{x}^{2}$, we have

$$
\frac{x}{r_{x}}=\mathcal{A}_{x} \cos \psi_{x}, \quad \frac{r_{x} x^{\prime}-r_{x}^{\prime} x}{\varepsilon_{x}}=\mathcal{A}_{x} \sin \psi_{x} .
$$

Adding the square of these equations, we obtain the Courant-Snyder invariant

$$
\left(\frac{x}{r_{x}}\right)^{2}+\left(\frac{r_{x} x^{\prime}-r_{x}^{\prime} x}{\varepsilon_{x}}\right)^{2}=\mathcal{A}_{x}^{2}=\text { const. }
$$

An analogous invariant holds in the $y$ plane.

The $x-x^{\prime}$ phase-space area enclosed by the ellipse defined by the Courant-Snyder invariant (B7) is $\pi \varepsilon_{x} \mathcal{A}_{x}$. A particle at the edge of the beam in phase space will have amplitude $\mathcal{A}_{x}=1$, showing that $\pi \varepsilon_{x}$ is the maximum phase-space area enclosed by particles in the coasting beam. A statistical average of Eq. (B7) shows that $\left\langle\mathcal{A}_{x}^{2}\right\rangle_{\perp}=1 / 2$ for consistency with the requirement that the statistical emittance of a KV beam satisfy $\varepsilon_{x}^{2}=$ $16\left[\left\langle x^{2}\right\rangle_{\perp}\left\langle x^{\prime 2}\right\rangle_{\perp}-\left\langle x x^{\prime}\right\rangle_{\perp}^{2}\right]$. For the $\mathrm{KV}$ distribution, $\varepsilon_{x}$ can be interpreted as the maximum single-particle emittance and a statistical edge measure of the rms-edge emittance (i.e., $\varepsilon_{x}=4 \varepsilon_{x \text {,rms }}$ ). Note that the KV distribution (16) is a delta function of $x$ - and the $y$-plane Courant-Snyder invariants that generates the required uniform-density elliptical beam required for self-consistency. The initial particle distributions defined in Sec. III D are based on linear-field Courant-Snyder invariants.

In the limit of zero space charge $(Q=0)$, the CourantSnyder invariant (B7) reduces to a form familiar from conventional accelerator physics of a single-particle oscillating in linear applied fields [97]. In this case it is conventional to employ alternative, positive-definite amplitude functions $\beta_{0 j}(s)$ (or alternatively, $w_{0 j}=\beta_{0 j}^{2}$ ) that are related to the $Q=0$ envelope functions $r_{j} \equiv r_{0 j}$ by

$$
r_{0 j}=\sqrt{\varepsilon_{j}} w_{0 j}=\sqrt{\varepsilon_{j} \beta_{0 j}}
$$

The function $\beta_{0 j}$ is called the betatron function, and for periodic lattices, is employed analogously to a defined special function that characterizes the applied-focusing properties of the lattice.

The Courant-Snyder invariant can be applied to improve the understanding of the pseudoequilibrium initial distributions defined in Sec. IV. Using the transformation (87) in the Courant-Snyder invariant (B7), we obtain

$$
\left(\frac{x}{r_{b}}\right)^{2}+\left(\frac{r_{b} x^{\prime}}{\varepsilon_{b}}\right)^{2}=\text { const }
$$

where $r_{b}$ and $\varepsilon_{b}$ are the envelope radius and emittance of an rms-equivalent, matched $\mathrm{KV}$ beam in a continuousfocusing channel. Adding the analogous $y$-plane invariant then shows that

$$
2 \frac{r_{b}^{2}}{\varepsilon_{b}^{2}}\left[\frac{1}{2} \mathbf{x}_{\perp}^{\prime 2}+\frac{\varepsilon_{b}^{2}}{2 r_{b}^{4}} \mathbf{x}_{\perp}^{2}\right]=\mathcal{A}_{x}^{2}+\mathcal{A}_{y}^{2}=\text { const. }
$$

For a matched KV equilibrium beam in a continuousfocusing channel, it is straightforward to show that the transverse beam Hamiltonian $H_{\perp}=\frac{1}{2} \mathbf{x}_{\perp}^{\prime 2}+\frac{k_{\beta 0}^{2}}{2} \mathbf{x}_{\perp}^{2}+$ $q \phi /\left(m \gamma_{b}^{2} \beta_{b}^{2} c^{2}\right)$ can be expressed as $H_{\perp}=\frac{1}{2} \mathbf{x}_{\perp}^{\prime 2}+\frac{\varepsilon_{b}^{2}}{2 r_{b}^{4}} \mathbf{x}_{\perp}^{2}$ [see Eq. (45)], giving

$$
2 \frac{r_{b}^{2}}{\varepsilon_{b}^{2}} H_{\perp}=\mathcal{A}_{x}^{2}+\mathcal{A}_{y}^{2}=\text { const. }
$$

This shows that the composite Courant-Snyder invariant $\mathcal{A}_{x}^{2}+\mathcal{A}_{y}^{2}$ is proportional to $H_{\perp}$ for the equivalent continuous-focusing channel. Therefore, the transforms applied to generate the pseudoequilibrium distributions from a continuous-focusing equilibrium distribution preserve linear-field Courant-Snyder invariants appropriate for the noncontinuous lattice. The transformations fail to produce an exact equilibrium because the self-fields are not linear for general (non-KV) continuous equilibrium distributions employed. For high space-charge intensity, the approximation of replacing the actual nonlinear spacecharge field with an rms-equivalent beam linear-field is expected to be worse for particles near the edge of the beam.

\section{APPENDIX C: RMS EQUIVALENCY AND PROJECTIONS OF THE DISTRIBUTIONS IN SEC. III D}

For a distribution to be rms equivalent with a KV distribution described by the envelope radii $r_{j}(j=x, y)$, the envelope angles $r_{j}^{\prime}$, and the rms-edge emittances $\varepsilon_{j}$, it follows from Eqs. (23) and (24) that the nonzero secondorder moments of the distribution must satisfy

$$
\begin{gathered}
\left\langle x^{2}\right\rangle_{\perp}=\frac{r_{x}^{2}}{4}, \quad\left\langle x x^{\prime}\right\rangle_{\perp}=\frac{r_{x} r_{x}^{\prime}}{4} \\
\left\langle x^{\prime 2}\right\rangle_{\perp}=\frac{r_{x}^{\prime 2}}{4}+\frac{\varepsilon_{x}^{2}}{4 r_{x}^{2}} .
\end{gathered}
$$

Here, we have expressed the $x$-plane equations and $\langle\cdots\rangle_{\perp}=\int d^{2} x \int d^{2} x^{\prime} \cdots f_{\perp} / \int d^{2} x \int d^{2} x^{\prime} f_{\perp}$. Analogous equations hold in the $y$ plane both here and in subsequent equations. All second-order cross moments must vanish (e.g., $\left.\langle x y\rangle_{\perp}=0\right)$.

To analyze constraints that rms equivalency places on the class of linear-field Courant-Snyder invariant (LCS) distributions defined by Eqs. (60)-(64), it is convenient to employ canonical transformations [30] by taking 


$$
X \equiv \sqrt{\varepsilon_{x}} \frac{x}{r_{x}}, \quad \dot{X} \equiv \sqrt{\varepsilon_{x}}\left(\frac{r_{x} x^{\prime}-r_{x}^{\prime} x}{\varepsilon_{x}}\right),
$$

with inverse transform

$$
x=\frac{r_{x}}{\sqrt{\varepsilon_{x}}} X, \quad x^{\prime}=\frac{\sqrt{\varepsilon_{x}}}{r_{x}} \dot{X}+\frac{r_{x}^{\prime}}{\sqrt{\varepsilon_{x}}} X .
$$

Phase-space area elements transform as

$$
\begin{aligned}
& d^{2} x_{\perp}=d x \otimes d y=\frac{r_{x} r_{y}}{\sqrt{\varepsilon_{x} \varepsilon_{y}}} d X \otimes d Y, \\
& d^{2} x_{\perp}^{\prime}=d x^{\prime} \otimes d y^{\prime}=\frac{\sqrt{\varepsilon_{x} \varepsilon_{y}}}{r_{x} r_{y}} d \dot{X} \otimes d \dot{Y},
\end{aligned}
$$

with

$$
\begin{aligned}
d^{2} x_{\perp} d^{2} x_{\perp}^{\prime} & =d x \otimes d y \otimes d x^{\prime} \otimes d y^{\prime} \\
& =d X \otimes d Y \otimes d \dot{X} \otimes d \dot{Y},
\end{aligned}
$$

reflecting the local phase-space invariance of a canonical transform [103]. Using these canonical transforms it is straightforward to show that the rms-equivalency requirements in Eq. (C1) can be expressed as

$$
\left\langle X^{2}\right\rangle_{\perp}=\left\langle\dot{X}^{2}\right\rangle_{\perp}=\frac{\varepsilon_{x}}{2}, \quad\langle X \dot{X}\rangle_{\perp}=0,
$$

and the linear-field Courant-Snyder invariant $\mathcal{A}^{2}$ in Eq. (61) becomes

$$
\mathcal{A}^{2}=\frac{1}{\varepsilon_{x}}\left(X^{2}+\dot{X}^{2}\right)+\frac{1}{\varepsilon_{y}}\left(Y^{2}+\dot{Y}^{2}\right),
$$

thereby simplifying the expression of the LCS distribution (60) to

$$
f_{\perp}\left(\mathcal{A}^{2}\right)=\frac{\lambda}{q} f\left(\frac{X^{2}+\dot{X}^{2}}{\varepsilon_{x}}+\frac{Y^{2}+\dot{Y}^{2}}{\varepsilon_{y}}\right) .
$$

Employing Eqs. (C5) and (C8), it follows by symmetry that $\langle X \dot{X}\rangle_{\perp}=0$ is satisfied independent of the form of the function $f$ used in the distribution definition (C8). Some straightforward manipulation using these equations then shows that $\left\langle X^{2}\right\rangle_{\perp}=\left\langle\dot{X}^{2}\right\rangle_{\perp}=\varepsilon_{x} / 2$ is satisfied if the function $f$ satisfies

$$
\frac{\int_{0}^{\infty} d U \int_{0}^{\infty} d \dot{U} U f(U+\dot{U})}{\int_{0}^{\infty} d U \int_{0}^{\infty} d \dot{U} f(U+\dot{U})}=\frac{1}{2} .
$$

Here, $U \equiv X^{2} / \varepsilon_{x}+Y^{2} / \varepsilon_{y}$ and $\dot{U} \equiv \dot{X}^{2} / \varepsilon_{x}+\dot{Y}^{2} / \varepsilon_{y}$. Denoting

$$
G(U) \equiv \int_{U}^{\infty} d \tilde{U} f(\tilde{U}),
$$

the constraint in Eq. (C9) can be equivalently expressed as

$$
\frac{\int_{0}^{\infty} d U U G(U)}{\int_{0}^{\infty} d U G(U)}=\frac{1}{2}
$$

This shows that the moment constraints $(\mathrm{C} 1)$ required for rms equivalency are automatically satisfied for LCS distributions defined by Eqs. (60) and (61) regardless of the (physical) values of $r_{j}, r_{j}^{\prime}$, and $\varepsilon_{j}$ and the specific form of the choice of function $f$.

Projections of the LCS distributions can be more easily calculated using the canonical transforms in Eqs. (C2)(C5). For example, the $x-y$ density projection reduces to

$$
\begin{aligned}
n & =\int d^{2} x_{\perp}^{\prime} f_{\perp} \\
& =\frac{\lambda}{q} \frac{\sqrt{\varepsilon_{x} \varepsilon_{y}}}{r_{x} r_{y}} \int d \dot{X} \int d \dot{Y} f\left(\frac{X^{2}+\dot{X}^{2}}{\varepsilon_{x}}+\frac{Y^{2}+\dot{Y}^{2}}{\varepsilon_{y}}\right) \\
& =\frac{\pi \lambda}{q} \frac{\varepsilon_{x} \varepsilon_{y}}{r_{x} r_{y}} \int_{\xi^{2}}^{\infty} d U f(U),
\end{aligned}
$$

where $\xi^{2} \equiv x^{2} / r_{x}^{2}+y^{2} / r_{y}^{2}$. Similarly, using

$$
d y \otimes d y^{\prime}=d Y \otimes d \dot{Y},
$$

the canonical transforms can be applied to calculate the $x-x^{\prime}$ phase-space projection as

$$
\begin{aligned}
\int d y \int d y^{\prime} f_{\perp} & =\frac{\lambda}{q} \int d Y \int d \dot{Y} f\left(\frac{X^{2}+\dot{X}^{2}}{\varepsilon_{x}}+\frac{Y^{2}+\dot{Y}^{2}}{\varepsilon_{y}}\right) \\
& =\frac{\pi \lambda \varepsilon_{y}}{q} \int_{\mathcal{A}_{x}^{2}}^{\infty} d U f(U),
\end{aligned}
$$

where $\mathcal{A}_{x}^{2} \equiv\left(x / r_{x}\right)^{2}+\left(r_{x} x^{\prime}-r_{x}^{\prime} x\right)^{2} / \varepsilon_{x}^{2}$. Further simplifications to Eqs. (C12) and (C14) can be made for specific choices of $f$ (see Table II).

\section{APPENDIX D: CONTINUOUS-FOCUSING WATERBAG EQUILIBRIUM DISTRIBUTION}

A thorough treatment of the waterbag equilibrium has been presented by Reiser [31] and others [32,59,67$69,104]$. Sheet beam models of the waterbag distribution have also been extensively studied in continuous and periodic-focusing channels both in terms of equilibrium $[105,106]$ and stability properties [106,107]. Here we review and extend analysis of the waterbag equilibrium within the present framework to facilitate generation of Vlasov simulation loads formulated with standard inputs for accelerator simulations. For a waterbag equilibrium distribution in continuous focusing, we take

$$
f_{\perp}\left(H_{\perp}\right)=f_{0} \Theta\left(H_{b}-H_{\perp}\right) .
$$

Here, $\Theta(x)$ is a unit-step function [see Eq. (57)], $f_{0}=$ const $>0$ is the distribution normalization factor, and $H_{b}=$ const is the value of the Hamiltonian $H_{\perp}$ at the physical beam edge at radius $r=r_{e}$, i.e.,

$$
\left.H_{\perp}\right|_{r=r_{e}}=H_{b} .
$$

The waterbag distribution expresses that all transverse particle energies out to the beam edge have uniform probability, which gives rise to the name "waterbag" motivated 
by analogy to an incompressible fluid confined within a membrane boundary. The sharp beam edge in phase space associated with the step-function definition of the distribution generates a simple, highly idealized model conducive to analytical calculations. Because $\partial f_{\perp}\left(H_{\perp}\right) / \partial H_{\perp}=$ $-f_{0} \delta\left(H_{b}-H_{\perp}\right) \leq 0$, the waterbag distribution is stable to all perturbations within the Vlasov model $[30,32]$.

Using the formulation developed in Sec. III B, we take $H_{\perp}=\mathbf{x}_{\perp}^{\prime 2} / 2+\psi$ with $\psi=k_{\beta 0}^{2} r^{2} / 2+q \phi /\left(m \gamma_{b}^{3} \beta_{b}^{2} c^{2}\right)$, and calculate the radial beam density $n=\int d^{2} x_{\perp}^{\prime} f_{\perp}$ using Eqs. (36) and (D1) to be

$$
n(r)=2 \pi f_{0} \begin{cases}H_{b}-\psi(r), & \psi<H_{b} \\ 0, & \psi>H_{b}\end{cases}
$$

Note that the density falls smoothly to zero at the physical beam edge [i.e., $n\left(r=r_{e}\right)=0$ ]. The physical edge radius $r_{e}$ is generally distinct from the rms-edge radius $r_{b}=$ $\sqrt{2\left\langle r^{2}\right\rangle_{\perp}}$ with $r_{e}>r_{b}$. Using Eq. (D3), the transformed Poisson equation (37) for $\psi$ can be expressed within the beam $\left(r<r_{e}\right)$ as

$$
\frac{1}{r} \frac{\partial}{\partial r}\left(r \frac{\partial \psi}{\partial r}\right)-k_{0}^{2} \psi=2 k_{\beta 0}^{2}-k_{0}^{2} H_{b}
$$

where

$$
k_{0}^{2} \equiv \frac{2 \pi q^{2} f_{0}}{\epsilon_{0} m \gamma_{b}^{3} \beta_{b}^{2} c^{2}}=\text { const. }
$$

Equation (D4) is a modified Bessel function equation of order zero [108]. The solution $\psi$ of this equation that is regular as $r \rightarrow 0$ and satisfies $\psi\left(r=r_{e}\right)=H_{b}$ is given within the beam by

$$
\psi(r)=H_{b}-2 \frac{k_{\beta 0}^{2}}{k_{0}^{2}}\left[1-\frac{I_{0}\left(k_{0} r\right)}{I_{0}\left(k_{0} r_{e}\right)}\right],
$$

where $I_{\ell}(x)$ is the modified Bessel function of order $\ell$. Using this result, Eq. (D3) for the density becomes

$$
\begin{aligned}
n(r) & =4 \pi f_{0} \frac{k_{\beta 0}^{2}}{k_{0}^{2}}\left[1-\frac{I_{0}\left(k_{0} r\right)}{I_{0}\left(k_{0} r_{e}\right)}\right] \\
& =\frac{2 \epsilon_{0} m \gamma_{b}^{2} \beta_{b}^{2} c^{2} k_{\beta 0}^{2}}{q^{2}}\left[1-\frac{I_{0}\left(k_{0} r\right)}{I_{0}\left(k_{0} r_{e}\right)}\right]
\end{aligned}
$$

within the beam. Similarly, the $x$-plane kinetic temperature $T_{x}=\left\langle x^{\prime 2}\right\rangle_{\mathbf{x}_{\perp}^{\prime}}$ is calculated, using Eq. (38) and previous results, to be

$$
T_{x}(r)=\frac{k_{\beta 0}^{2}}{k_{0}^{2}}\left[1-\frac{I_{0}\left(k_{0} r\right)}{I_{0}\left(k_{0} r_{e}\right)}\right]
$$

within the beam. Comparing Eqs. (D7) and (D8), note that $T_{x}(r) \propto n(r)$. This proportionality between $T_{x}$ and $n$ is a consequence of the waterbag equilibrium choice for $f_{\perp}\left(H_{\perp}\right)$, and is not a general result for continuous-focusing equilibria.

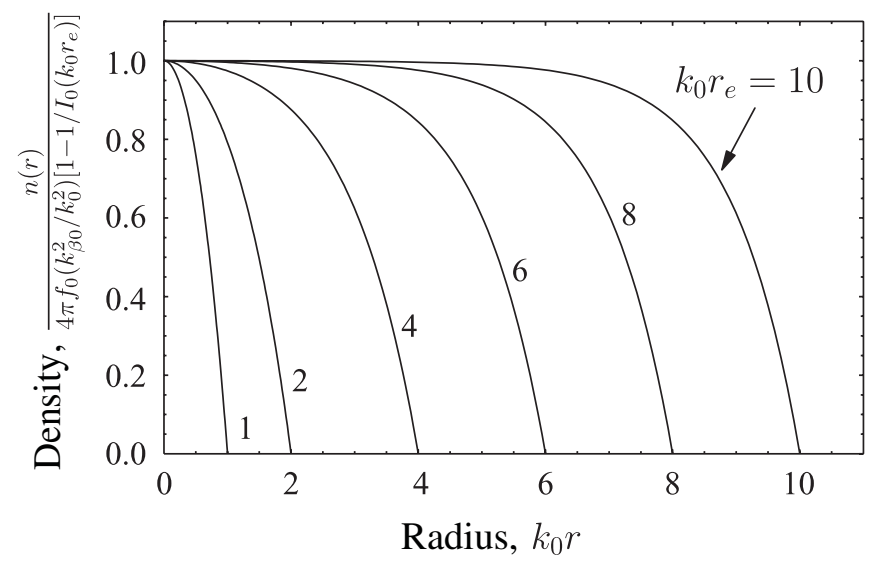

FIG. 10. For a waterbag equilibrium, the scaled density profile $n(r) /\left\{4 \pi f_{0}\left(k_{\beta 0}^{2} / k_{0}^{2}\right)\left[1-1 / I_{0}\left(k_{0} r_{e}\right)\right]\right\}$ is plotted versus the scaled radial coordinate $k_{0} r$, calculated from Eq. (D7) for the indicated values of $k_{0} r_{e}$.

In Fig. 10 the normalized waterbag density profile is plotted as a function of $k_{0} r$ for characteristic values of $k_{0} r_{e}$. Note that as $k_{0} r_{e}$ increases, the density profile (and the temperature profile with $T_{x} \propto n$ ) becomes increasingly flat within the core of the beam, with $r \ll r_{e}$.

It can be useful to employ $H_{\perp}=\frac{1}{2} \mathbf{x}_{\perp}^{22}+\psi$ [see Eq. (35)] and Eq. (D6) for $\psi$ to explicitly calculate the waterbag distribution as

$$
f_{\perp}\left(\mathbf{x}_{\perp}, \mathbf{x}_{\perp}^{\prime}\right)=f_{0} \Theta\left(2 \frac{k_{\beta 0}^{2}}{k_{0}^{2}}\left[1-\frac{I_{0}\left(k_{0} r\right)}{I_{0}\left(k_{0} r_{e}\right)}\right]-\frac{1}{2} \mathbf{x}_{\perp}^{\prime 2}\right) .
$$

Note that $H_{b}$ has been eliminated in Eq. (D9), and the distribution is expressed in terms of normalization factor $f_{0}$, the scaled edge radius $k_{0} r_{e}$, and $k_{\beta 0} / k_{0}$.

To use the formulation above effectively, distribution parameters should be cast in terms of standard quantities associated with accelerator physics as discussed in Sec. III B. First, the beam line charge can be calculated using $\lambda=2 \pi q \int_{0}^{r_{e}} d r r n(r)$ and Eq. (D7) to show that

$$
\begin{aligned}
\lambda & =4 \pi^{2} q f_{0} \frac{k_{\beta 0}^{2}}{k_{0}^{2}} r_{e}^{2}\left[1-\frac{2}{k_{0} r_{e}} \frac{I_{1}\left(k_{0} r_{e}\right)}{I_{0}\left(k_{0} r_{e}\right)}\right] \\
& =4 \pi^{2} q f_{0} \frac{k_{\beta 0}^{2}}{k_{0}^{2}} r_{e}^{2} \frac{I_{2}\left(k_{0} r_{e}\right)}{I_{0}\left(k_{0} r_{e}\right)} .
\end{aligned}
$$

Here we have employed the modified Bessel function identities [108]

$$
\begin{aligned}
\frac{d}{d x}\left[x^{\ell} I_{\ell}(x)\right] & =x^{\ell} I_{\ell-1}(x), \\
-\frac{2 \ell}{x} I_{\ell}(x) & =I_{\ell+1}(x)-I_{\ell-1}(x),
\end{aligned}
$$

with $\ell$ an integer, to simplify the integrals in the calculation of $\lambda$. Similarly, the statistical rms-beam envelope given by $r_{b}=\sqrt{2\left\langle r^{2}\right\rangle_{\perp}}$ with $\left\langle r^{2}\right\rangle_{\perp}=\int_{0}^{r_{e}} d r r^{3} n(r) / \int_{0}^{r_{e}} d r r n(r)$ can 
be explicitly calculated using Eq. (D7) [or equivalently, using Eq. (40)] to be

$$
\left(\frac{r_{b}}{r_{e}}\right)^{2}=\frac{I_{0}\left(k_{0} r_{e}\right)}{I_{2}\left(k_{0} r_{e}\right)}-\frac{4}{\left(k_{0} r_{e}\right)^{2}}\left[2+\left(k_{0} r_{e}\right) \frac{I_{3}\left(k_{0} r_{e}\right)}{I_{2}\left(k_{0} r_{e}\right)}\right] .
$$

From Eqs. (D5) and (D10), the perveance $Q=$ $q \lambda /\left(2 \pi \epsilon_{0} m \gamma_{b}^{3} \beta_{b}^{2} c^{2}\right)$ is conveniently expressed as

$$
Q=\left(k_{\beta 0} r_{e}\right)^{2} \frac{I_{2}\left(k_{0} r_{e}\right)}{I_{0}\left(k_{0} r_{e}\right)} .
$$

Then Eqs. (D11) and (D12) can be combined to obtain the constraint equation

$$
\begin{aligned}
\frac{k_{\beta 0}^{2} r_{b}^{2}}{Q}= & \frac{I_{0}^{2}\left(k_{0} r_{e}\right)}{I_{2}^{2}\left(k_{0} r_{e}\right)} \\
& -\frac{4}{\left(k_{0} r_{e}\right)^{2}}\left[2 \frac{I_{0}\left(k_{0} r_{e}\right)}{I_{2}\left(k_{0} r_{e}\right)}+\left(k_{0} r_{e}\right) \frac{I_{0}\left(k_{0} r_{e}\right) I_{3}\left(k_{0} r_{e}\right)}{I_{2}^{2}\left(k_{0} r_{e}\right)}\right],
\end{aligned}
$$

which relates the dimensionless factor $k_{0} r_{e}$ in terms of the dimensionless ratio of beam physics parameters $k_{\beta 0}^{2} r_{b}^{2} / Q$. Using Eq. (47), $k_{\beta 0}^{2} r_{b}^{2} / Q$ [or $k_{0} r_{e}$ using Eq. (D13)] can be directly related to the rms-equivalent beam measure of relative space-charge strength $\sigma / \sigma_{0}$ as

$$
\frac{k_{\beta 0}^{2} r_{b}^{2}}{Q}=\frac{1}{1-\left(\sigma / \sigma_{0}\right)^{2}} .
$$

Alternatively, the dimensionless self-field parameter $s_{b}$ defined in Eq. (48) can be related to $k_{0} r_{e}$ from

$$
s_{b} \equiv \frac{\hat{\omega}_{p}^{2}}{2 \gamma_{b}^{3} \beta_{b}^{2} c^{2} k_{\beta 0}^{2}}=1-\frac{1}{I_{0}\left(k_{0} r_{e}\right)} .
$$

Here, $\hat{\omega}_{p}=\sqrt{q^{2} \hat{n} /\left(\epsilon_{0} m\right)}$ is the plasma frequency defined from the peak, on-axis beam density $\hat{n}=n(r=0)$. For specified $Q$ the ratio of $k_{0} / k_{\beta 0}$ can be calculated from Eq. (D12) and $k_{0} r_{e}$ as

$$
\frac{k_{\beta 0}}{k_{0}}=\frac{1}{\left(k_{0} r_{e}\right)} \sqrt{Q \frac{I_{0}\left(k_{0} r_{e}\right)}{I_{2}\left(k_{0} r_{e}\right)}} .
$$

The matched-beam envelope constraint [see Eq. (39)]

$$
k_{\beta 0}^{2} r_{b}-\frac{Q}{r_{b}}-\frac{\varepsilon_{b}^{2}}{r_{b}^{3}}=0
$$

can be employed in the constraint equation (D13) to eliminate either $k_{\beta 0}^{2}, r_{b}$, or $Q$ occurring in $k_{\beta 0}^{2} r_{b}^{2} / Q$ in terms of the emittance $\varepsilon_{b}$ to affect various parametrization choices.

The nonlinear constraint equation (D13) must, in general, be solved numerically to specify the needed value of $k_{0} r_{e}$. Using Eqs. (D13) and (D14), $k_{0} r_{e}$ can be regarded as a function of the rms-equivalent beam tune depression $\sigma / \sigma_{0}$. These equations are solved numerically to plot $k_{0} r_{e}$ as a function of $\sigma / \sigma_{0}$ in Fig. 11. Because $k_{0} r_{e}$ is a

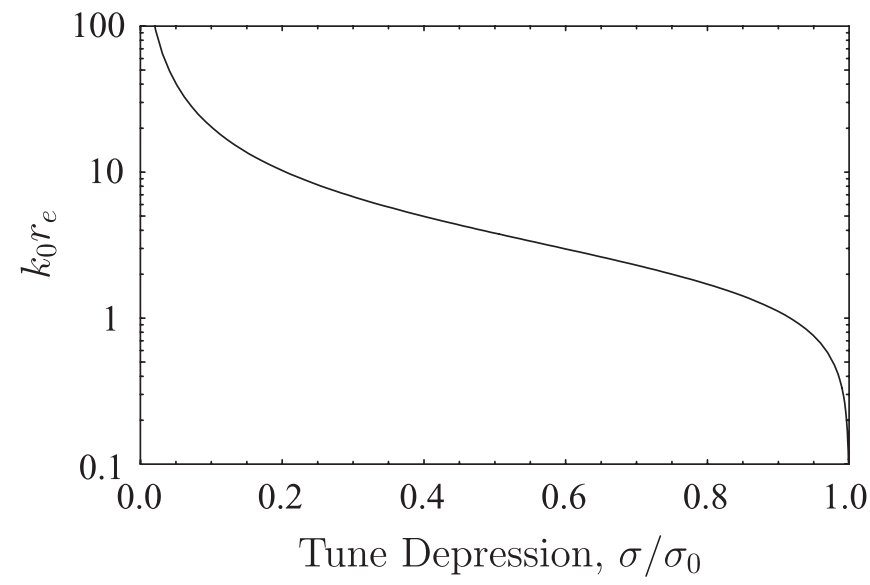

FIG. 11. Waterbag equilibrium parameter $k_{0} r_{e}$ versus rmsequivalent beam tune depression $\sigma / \sigma_{0}$ as calculated from Eqs. (D13) and (D14).

one-to-one function of $\sigma / \sigma_{0}$, the relative space-charge strength can be regarded as uniquely determining $k_{0} r_{e}$. Figure 11 illustrates the wide range of characteristic values of $k_{0} r_{e}$ obtainable as the relative space-charge strength is varied. From the envelope equation, note that $k_{\beta 0}^{2} r_{b}^{2} / Q=$ $1+\varepsilon_{b}^{2} /\left(Q r_{b}^{2}\right)>1$. Analysis of Eq. (D13) shows that $k_{\beta 0}^{2} r_{b}^{2} / Q$ is a monotonic decreasing function of $k_{0} r_{e}$ with $\lim _{k_{0} r_{e} \rightarrow 0} k_{\beta 0}^{2} r_{b}^{2} / Q \rightarrow \infty \quad$ and $\quad \lim _{k_{0} r_{e} \rightarrow \infty} k_{\beta 0}^{2} r_{b}^{2} / Q=1$. Therefore, a unique value of $k_{0} r_{e} \in(0, \infty)$ exists for any equilibrium with finite space charge $(Q \neq 0)$. Analytical solution of the constraint equation (D13) is possible in the limit of small and large values of $k_{0} r_{e}$. Using the expansion [108]

$$
I_{\ell}(x)=\sum_{k=0}^{\infty} \frac{(x / 2)^{\ell+2 k}}{k !(\ell+k) !}
$$

for $|x| \ll 1$, we obtain to leading order

$$
k_{0} r_{e} \simeq \frac{4}{\sqrt{3\left(k_{\beta 0}^{2} r_{b}^{2} / Q-17 / 18\right)}}
$$

for values of $k_{\beta 0}^{2} r_{b}^{2} / Q$ sufficiently large to produce small $k_{0} r_{e}$. This limit of small $k_{\beta 0}^{2} r_{b}^{2} / Q$ corresponds to weak space-charge forces relative to applied-focusing forces $\left(\sigma / \sigma_{0} \sim 1\right)$ and Eqs. (D6) and (D7) for $n(r)$ can be approximated in this regime as

$$
n(r) \simeq \pi f_{0} k_{\beta 0}^{2}\left(r_{e}^{2}-r^{2}\right)
$$

within the beam. Similar parabolic approximations follow immediately for $\psi$ and $T_{x}$ in this limit. Using the expansion [108]

$$
\begin{aligned}
I_{\ell}(x)= & \frac{e^{x}}{\sqrt{2 \pi x}}\left[1+\frac{(-1)^{1}\left(4 \ell^{2}-1^{2}\right)}{1 !(8 x)^{1}}\right. \\
& \left.+\frac{(-1)^{2}\left(4 \ell^{2}-1^{2}\right)\left(4 \ell^{2}-3^{2}\right)}{2 !(8 x)^{2}} \cdots\right]
\end{aligned}
$$


for $x \gg \ell$, the constraint equation (D13) can be approximated to leading order as

$$
k_{0} r_{e} \simeq \frac{2}{\sqrt{k_{\beta 0}^{2} r_{b}^{2} / Q-1}}
$$

for values of $k_{\beta 0}^{2} r_{b}^{2} / Q>1$ sufficiently close to unity to produce large $k_{0} r_{e}$. The large- $k_{0} r_{e}$ regime corresponds to strong space-charge depression with $\sigma / \sigma_{0}$ small. Simplified expressions for the density profile valid within the full radial range of the beam core are more difficult to derive in this case. For general space-charge strength, the limiting solutions in Eqs. (D18) and (D20) can be employed to seed numerical solutions of the constraint equation (D13) using conventional root-finding techniques [95].

Although the envelope equation (39) can be applied to calculate the beam rms-edge emittance $\varepsilon_{b}$ in terms of $k_{\beta 0}^{2}$, $r_{b}$, and $Q$, it can be useful in some circumstances to calculate $\varepsilon_{b}=\varepsilon_{j}$ explicitly for the waterbag distribution function (D1). From Eqs. (42) and (D1), $\varepsilon_{b}^{2}=2 r_{b}^{2}\left\langle\mathbf{x}_{\perp}^{\prime 2}\right\rangle_{\perp}$ can be calculated to be

$\varepsilon_{b}^{2}=\frac{16 \pi^{2} q f_{0} r_{b}^{2}}{\lambda}\left[\frac{H_{b}^{2} r_{e}^{2}}{4}-H_{b} \int_{0}^{r_{e}} d r r \psi+\frac{1}{2} \int_{0}^{r_{e}} d r r \psi^{2}\right]$.

Use of Eqs. (D6) and (D10) in this result leads to

$$
\begin{aligned}
\varepsilon_{b}^{2}= & 4 r_{b}^{2} \frac{k_{\beta 0}^{2}}{k_{0}^{2}}\left[2 \frac{I_{0}\left(k_{0} r_{e}\right)}{I_{2}\left(k_{0} r_{e}\right)}-\frac{4}{\left(k_{0} r_{e}\right)} \frac{I_{1}\left(k_{0} r_{e}\right)}{I_{2}\left(k_{0} r_{e}\right)}\right. \\
& \left.-\frac{I_{1}^{2}\left(k_{0} r_{e}\right)}{I_{0}\left(k_{0} r_{e}\right) I_{2}\left(k_{0} r_{e}\right)}\right] .
\end{aligned}
$$

To better understand properties of the waterbag equilibrium, we employ Eqs. (D11)-(D17) to plot the radial density profile and the phase-space boundary of the distribution in Fig. 12 for fixed applied-focusing strength $\left(k_{\beta 0}^{2}=\right.$ const) and fixed beam perveance ( $Q=$ const) as the relative space-charge strength $\left(\sigma / \sigma_{0}\right)$ is varied. In Fig. 12(a) the scaled radial density profile is plotted. For the waterbag equilibrium the temperature profile is proportional to the density profile [i.e., $T_{x} \propto n$, see Eqs. (D7) and (D8)], and therefore Fig. 12(a) also serves to illustrate the beam radial temperature profile. The boundary edge of the waterbag equilibrium distribution in $\mathbf{x}_{\perp}-\mathbf{x}_{\perp}^{\prime}$ phase-space is shown in Fig. 12(b). This $f_{\perp}=0$ boundary is calculated as the maximum value of $\mathbf{x}_{\perp}^{\prime}$ as a function of $r$ from Eq. (D9) to be

$$
\operatorname{Max}\left(\mathbf{x}_{\perp}^{\prime}\right)=2 \frac{k_{\beta 0}}{k_{0}}\left[1-\frac{I_{0}\left(k_{0} r\right)}{I_{0}\left(k_{0} r_{e}\right)}\right]^{1 / 2}
$$

within the beam. The distribution $f_{\perp}$ is uniformly filled within the outer edge. Various dimensionless parameters for the equilibria in Fig. 12 are given in Table III. Note that for high space-charge intensity (small $\sigma / \sigma_{0}$ ) the waterbag equilibrium density profile becomes very flat deep within
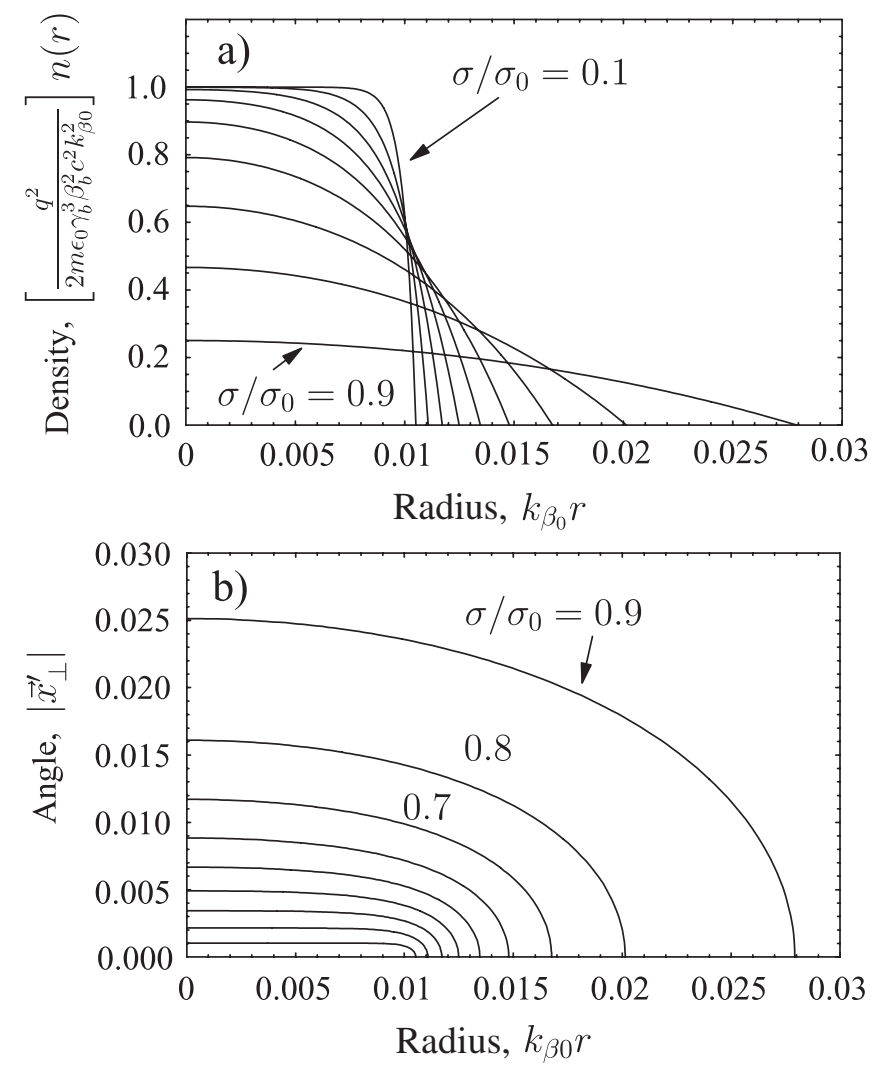

FIG. 12. Waterbag equilibrium distribution in continuousfocusing channel for fixed focusing-field strength $\left(k_{\beta 0}^{2}=\right.$ const) and perveance $Q=10^{-4}$ with (rms-equivalent beam measure) relative space-charge strengths $\sigma / \sigma_{0}=0.9,0.8, \ldots$, 0.1 . In (a) the scaled density profile $\left[q^{2} /\left(2 m \epsilon_{0} \gamma_{b}^{3} \beta_{b}^{2} c^{2} k_{\beta 0}^{2}\right)\right] n(r)$ is plotted versus the dimensionless radial coordinate $k_{\beta 0} r$, and in (b) the distribution edge $\left(f_{\perp}=0\right.$ curve $)$ in $\mathbf{x}_{\perp}-\mathbf{x}_{\perp}^{\prime}$ phase space is plotted as a function of $k_{\beta 0} r$ and $\left|\mathbf{x}_{\perp}^{\prime}\right|$. Values of $\sigma / \sigma_{0}$ correspond to the dimensionless equilibrium parameters in Table III.

in the core $\left(r \ll r_{e}\right)$ due to Debye screening effects associated with the interaction of the applied-focusing and space-charge forces [30,109]. Near the edge $\left(r \lesssim r_{e}\right)$ the applied-focusing forces start to dominate the self-field forces and the density decreases rapidly to zero with a characteristic (modified Bessel function) falloff associated with the waterbag equilibrium choice. For weak relative space-charge forces $\left(\sigma / \sigma_{0} \sim 1\right)$, the density profile approaches the parabolic limiting form in Eq. (D19), and the phase-space boundary becomes elliptical [i.e., Eq. (D22) is approximated by $\left[\operatorname{Max}\left(\mathbf{x}_{\perp}^{\prime}\right)\right]^{2}+k_{\beta 0}^{2} r_{e}^{2}=$ $\left.k_{\beta 0}^{2} r_{e}^{2}\right]$. For large relative space-charge intensity $\left(\sigma / \sigma_{0} \ll\right.$ $1)$, the phase-space boundary of the uniform core distribution becomes more rectangular, indicating nearly forcefree motion deep within the beam core until a particle enters the edge region where a strong nonlinear force transition effectively reflects the particle. From Table III, note that small values of $\sigma / \sigma_{0}$ correspond to values of the self-field parameter $s_{b}$ that are extremely close to $s_{b}=1$. 
TABLE III. Dimensionless waterbag equilibrium parameters in Fig. 12 calculated for specified $\sigma / \sigma_{0}$. The values of $k_{0} / k_{\beta 0}$ and $k_{\beta 0} \varepsilon_{b}$ are evaluated for $Q=10^{-4}$, and all other quantities are independent of $Q$.

\begin{tabular}{l|cccc|ccc}
\hline \hline & & & & \multicolumn{3}{c}{$Q=10^{-4}$} \\
$\sigma / \sigma_{0}$ & $k_{0} r_{e}$ & $s_{b}$ & $\frac{k_{\beta 0}^{2} r_{b}^{2}}{Q}$ & $\frac{r_{e}}{r_{b}}$ & $\frac{k_{0}}{k_{\beta 0}}$ & $10^{3} \times k_{\beta 0} r_{b}$ & $10^{3} \times k_{\beta 0} \varepsilon_{b}$ \\
\hline 0.9 & 1.112 & 0.2502 & 0.19 & 1.217 & 39.81 & 22.94 & 0.4737 \\
0.8 & 1.709 & 0.4666 & 0.36 & 1.208 & 84.87 & 16.67 & 0.2222 \\
0.7 & 2.304 & 0.6477 & 0.51 & 1.197 & 137.5 & 14.00 & 0.1373 \\
0.6 & 2.979 & 0.7916 & 0.64 & 1.183 & 201.5 & 12.50 & 0.09375 \\
0.5 & 3.821 & 0.8968 & 0.75 & 1.166 & 283.8 & 11.55 & 0.06667 \\
0.4 & 4.978 & 0.9626 & 0.84 & 1.144 & 398.7 & 10.91 & 0.04762 \\
0.3 & 6.789 & 0.9928 & 0.91 & 1.118 & 579.3 & 10.48 & 0.03297 \\
0.2 & 10.25 & 0.9997 & 0.96 & 1.085 & 925.6 & 10.21 & 0.02083 \\
0.1 & 20.38 & 0.99999998 & 0.99 & 1.046 & 1938.0 & 10.05 & 0.01010 \\
\hline \hline
\end{tabular}

Thus, the self-field parameter is insensitive relative to $k_{0} r_{e}$ to employ to specify scaled intense-beam waterbag equilibria with high space-charge intensity.

To load the waterbag equilibrium distribution in either direct-Vlasov or PIC simulations, the general framework presented in Sec. III B can be applied. For PIC loading of the waterbag distribution, the radial probability transform (51) for loading macroparticle coordinates $\mathbf{x}_{\perp}$ can be expressed in the reduced form

$$
\frac{r}{r_{e}}\left[\frac{r}{r_{e}} \frac{I_{0}\left(k_{0} r_{e}\right)}{I_{2}\left(k_{0} r_{e}\right)}-\frac{1}{k_{0} r_{e}} \frac{I_{1}\left(k_{0} r\right)}{I_{2}\left(k_{0} r_{e}\right)}\right]=\hat{u}_{r} .
$$

Here, $\hat{u}_{r} \in[0,1)$ is an independent, uniformly distributed random number generated for each macroparticle. This equation must, in general, be solved numerically for $r\left(\hat{u}_{r}\right)$ to specify macroparticle coordinates using Eq. (52). Values can be saved on a radial grid in $r \in\left[0, r_{e}\right]$, and interpolation applied to efficiently load many macroparticles. For loading the macroparticle angles $\mathbf{x}_{\perp}^{\prime}$, the probability transform (53) can be greatly simplified by exploiting the structure of the waterbag distribution. With particle radii $r=\left|\mathbf{x}_{\perp}\right|$ specified, the macroparticle angles $\left|\mathbf{x}_{\perp}^{\prime}\right|$ are uniformly distributed in $U=\frac{1}{2} \mathbf{x}_{\perp}^{\prime 2}$ from $U=0$ to a maximum value consistent with Eq. (D22) leading to

$$
U\left(\hat{u}_{U}\right)=2 \frac{k_{\beta 0}^{2}}{k_{0}^{2}}\left[1-\frac{I_{0}\left(k_{0} r\right)}{I_{0}\left(k_{0} r_{e}\right)}\right] \hat{u}_{U} .
$$

Here, $\hat{u}_{U} \in[0,1)$ is an independent, uniformly distributed random number generated for each macroparticle. Macroparticle angles are set using this value of $U\left(\hat{u}_{U}\right)$ in Eq. (54).

\section{APPENDIX E: CONTINUOUS-FOCUSING PARABOLIC EQUILIBRIUM DISTRIBUTION}

For a parabolic equilibrium distribution in continuous focusing, we take

$$
f_{\perp}\left(H_{\perp}\right)=f_{0}\left(H_{b}-H_{\perp}\right) \Theta\left(H_{b}-H_{\perp}\right),
$$

where $\Theta(x)$ is a unit-step function [see Eq. (57)], $f_{0}=$ const $>0$ is the distribution normalization factor, and $H_{b}=$ const is the value of the Hamiltonian $H_{\perp}$ at the physical beam edge at radius $r=r_{e}$, i.e.,

$$
\left.H_{\perp}\right|_{r=r_{e}}=H_{b} .
$$

The parabolic distribution has linearly decreasing particle probabilities with increasing transverse particle energy out to a sharp beam edge where the probability is zero. This distribution is named "parabolic" because at fixed $\mathbf{x}_{\perp}$, the probability decreases parabolically with increasing $\mathbf{x}_{\perp}^{\prime}$ due to the $\frac{1}{2} \mathbf{x}^{\prime 2}$ dependence of $H_{\perp}$ on $\mathbf{x}_{\perp}^{\prime}$. The parabolic distribution coarsely reflects what one might expect on physical grounds - that probabilities fall off towards the edge of the beam in a continuous manner, and may in this sense represent a lesser degree of idealization than the waterbag distribution [see Sec. D].

Using the formulation developed in Sec. III B, we take $H_{\perp}=\mathbf{x}_{\perp}^{\prime 2} / 2+\psi \quad$ with $\psi=k_{\beta 0}^{2} r^{2} / 2+q \phi /\left(m \gamma_{b}^{3} \beta_{b}^{2} c^{2}\right)$ and calculate the radial beam density profile $n(r)=$ $\int d^{2} x_{\perp}^{\prime} f_{\perp}$ from Eqs. (E1) and (36). This gives

$$
n(r)=\pi f_{0} \begin{cases}{\left[H_{b}-\psi(r)\right]^{2},} & \psi<H_{b}, \\ 0, & \psi>H_{b} .\end{cases}
$$

Using Eq. (E3), the transformed Poisson equation (37) can be conveniently expressed within the beam $\left(r<r_{e}\right)$ as

$$
\frac{1}{r} \frac{\partial}{\partial r}\left(r \frac{\partial \psi}{\partial r}\right)+\frac{K}{2}\left(H_{b}-\psi\right)^{2}=2 k_{\beta 0}^{2} r_{e}^{2}
$$

Here,

$$
K \equiv \frac{2 \pi q^{2} f_{0} H_{b} r_{e}^{2}}{\epsilon_{0} m \gamma_{b}^{3} \beta_{b}^{2} c^{2}}=\mathrm{const}
$$

is a dimensionless constant. Equation (E4) can be integrated subject to 


$$
\begin{gathered}
\psi\left(r=r_{e}\right)=H_{b},\left.\quad \frac{\partial \psi}{\partial r}\right|_{r=0}=0, \\
\left.\frac{\partial \psi}{\partial r}\right|_{r=r_{e}}=k_{\beta 0}^{2} r_{e}-\frac{Q}{r_{e}} .
\end{gathered}
$$

Here, $\psi\left(r=r_{e}\right)=H_{b}$ follows from Eq. (E2), the derivative condition on $\psi$ at $r=0$ follows from the structure of Eq. (E4), and the derivative condition on $\psi$ at $r=r_{e}$ is readily derived from a direct integration on the equilibrium Poisson equation (33) to show that $\partial \phi /\left.\partial r\right|_{r=r_{e}}=$ $-\lambda /\left(2 \pi \epsilon_{0} r_{e}\right)$ and employing the definition of the perveance $Q=q \lambda /\left(2 \pi \epsilon_{0} m \gamma_{b}^{3} \beta_{b}^{2} c^{2}\right)=$ const.

Equation (E4) for $\psi$ is highly nonlinear and must be numerically integrated subject to the conditions in Eq. (E6). The integration can be carried out inward from $r=r_{e}$ and subject to the two "initial" conditions in Eq. (E6) at $r=r_{e}$, i.e., $\psi\left(r=r_{e}\right)=H_{b} \quad$ and $[\partial \psi / \partial r]_{r=r_{e}}=k_{\beta 0}^{2} r_{e}-Q / r_{e}$. Only specific parameters will be consistent with the derivative condition $[\partial \psi / \partial r]_{r=0}=0$ in Eq. (E6) necessary for a physical solution. This boundary condition can in this sense be employed as a constraint to eliminate one free parameter. Careful analysis of Eqs. (E4) and (E6) shows that simple rescalings result in a final system with three free parameters, one of which can be regarded as eliminated in enforcing boundary conditions. Ultimate specification of the solution in terms of standard quantities associated with accelerator physics as discussed in Sec. III B will, in general, become complicated to enforce even in numerical solution.

Fortunately, a more convenient alternative formulation can be derived as follows. Within the beam $\left(r<r_{e}\right)$, Eq. (E3) can used to relate $\psi$ to $n$ by

$$
\psi=H_{b}\left(1-\sqrt{\frac{n}{\pi f_{0} H_{b}^{2}}}\right) .
$$

This result, together with a simple variable rescaling

$$
r \equiv r_{s} \rho, \quad n \equiv \hat{n} N,
$$

where $r_{s}>0$ is a scale radius to be determined, and $\hat{n}=$ $n(r=0)>0$ is the on-axis density $[N(\rho=0)=1]$, can then be applied to express Eq. (E4) to give

$$
N \frac{\partial^{2} N}{\partial \rho^{2}}+\frac{N}{\rho} \frac{\partial N}{\partial \rho}-\frac{1}{2}\left(\frac{\partial N}{\partial \rho}\right)^{2}=-C_{1} N^{3 / 2}+C_{2} N^{5 / 2} .
$$

Here,

$$
\begin{aligned}
C_{1} & =4 k_{\beta 0}^{2} r_{s}^{2} \sqrt{\frac{\pi f_{0}}{\hat{n}}}>0, \\
C_{2} & =\frac{1}{2} \frac{q^{2} \hat{n}}{m \epsilon_{0}} \frac{C_{1}}{\gamma_{b}^{3} \beta_{b}^{2} c^{2} k_{\beta 0}^{2}}>0 .
\end{aligned}
$$

The freedom of scale choice in $r_{s}$ allows us to take $C_{1}=1$. Then we identify

$$
C_{2}=\frac{\hat{\omega}_{p}^{2}}{2 \gamma_{b}^{3} \beta_{b}^{2} c^{2} k_{\beta 0}^{2}} \equiv s_{b},
$$

where $\hat{\omega}_{p}=\sqrt{q^{2} \hat{n} /\left(m \epsilon_{0}\right)}$ is the on-axis plasma frequency, and $s_{b}$ is the dimensionless self-field parameter defined in Eq. (48).

With this rescaling, the normalized density $N$ within the beam is given by

$$
N \frac{\partial^{2} N}{\partial \rho^{2}}+\frac{N}{\rho} \frac{\partial N}{\partial \rho}-\frac{1}{2}\left(\frac{\partial N}{\partial \rho}\right)^{2}=-N^{3 / 2}+s_{b} N^{5 / 2},
$$

subject to

$$
N(\rho=0)=1,\left.\quad \frac{\partial N}{\partial \rho}\right|_{\rho=0}=0 .
$$

Equation (E12) can be simply integrated outward from the initial conditions at $\rho=0$ in Eq. (E13) until the beam edge is reached at $\rho=\rho_{e}$, where

$$
N\left(\rho=\rho_{e}\right)=0 .
$$

It also follows directly from Eq. (E12) that

$$
\left.\frac{\partial N}{\partial \rho}\right|_{\rho=\rho_{e}}=0 .
$$

Note that in this formulation only one dimensionless parameter $s_{b}>0$ is necessary to specify the normalized density $N$ of the parabolic equilibrium distribution.

As a practical matter, the numerical integration for $N$ needs to be started from a small value of $\rho \neq 0$. A powerseries analysis of Eq. (E12) shows that the first few terms of the solution for small $\rho$ are given by

$$
\begin{aligned}
N= & 1-\frac{1-s_{b}}{4} \rho^{2}+\frac{\left(1-s_{b}\right)\left(1-2 s_{b}\right)}{64} \rho^{4} \\
& +\frac{s_{b}\left(1-s_{b}\right)\left(11-13 s_{b}\right)}{4608} \rho^{6}+\cdots .
\end{aligned}
$$

For consistency with $\partial N / \partial \rho<0$ for small $\rho$, this expansion shows that the physical range of the self-field parameter $s_{b}$ for the parabolic equilibrium is $s_{b} \in[0,1)$. The limit $s_{b}=0$ corresponds to zero space-charge intensity with a shaped density profile reaching into the core of the beam (the analysis below shows that the density expansion truncates at the first two terms), and $s_{b} \rightarrow 1$ corresponds to the limit of maximum space-charge intensity with a flat density profile in the core of the beam.

Numerical solutions of Eqs. (E12) and (E13) for $N$ versus $\rho$ are plotted in Fig. 13 for values of $s_{b} \in[0,1)$. Because of an extreme sensitivity of the solution in $s_{b}$ near $s_{b}=1$, we employ an alternative parameter,

$$
p \equiv-\log \left(1-s_{b}\right)
$$

to characterize the solutions. The solutions are plotted out to the beam edge $\rho=\rho_{e}$ where $N=0$. In generating these solutions, it is convenient to integrate through $N=0$ to 


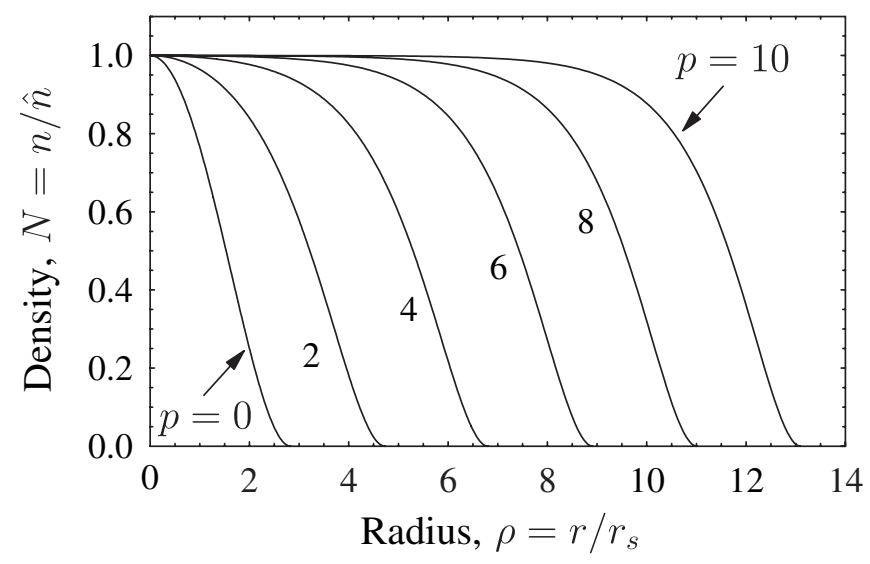

FIG. 13. For a parabolic equilibrium, the scaled density $N=$ $n / \hat{n}$ is plotted versus the scaled radial coordinate $\rho=r / r_{s}$ numerically calculated from Eqs. (E12) and (E13) for $p=$ $0,2, \ldots, 10$.

allow calculation of $\rho_{e}$ by numerical root finding. This can be accomplished by replacing $N^{3 / 2} \rightarrow N|N|^{1 / 2}$ and $N^{5 / 2} \rightarrow|N|^{5 / 2}$ on the right-hand side of Eq. (E12) without influencing the needed core solution of $N(\rho)$ for $\rho<\rho_{e}$. The extended solution for $N$ with $\rho>\rho_{e}$ has $N \geq 0$ and generally oscillates in $\rho$ between zero and a value at some fraction of the core. Because $\rho_{e}$ occurs where $\partial N / \partial \rho=0$, $\rho_{e}$ can be calculated by bracketed numerical root finding for $\partial N(\rho) / \partial \rho=0$ near the first radial location where $N \simeq 0$.

Note from the solutions in Fig. 13 that the core beam density profile becomes flat as $s_{b} \rightarrow 1$ (i.e., $p \rightarrow \infty$ ) out until $\rho$ increases towards $\rho_{e}$ where $N$ drops to zero with a radial profile characteristic of the parabolic equilibrium choice of $f_{\perp}\left(H_{\perp}\right)$. This edge shape extends deeper into the core of the beam as $s_{b}$ (or $p$ ) decreases. In the limit $s_{b}=p=0$, the exact solution to Eqs. (E12) satisfying Eq. (E13) is

$$
N=\left(1-\frac{\rho^{2}}{8}\right)^{2}
$$

with a corresponding beam edge (i.e., where $N=0$ ) at $\rho=\rho_{e}=2 \sqrt{2} \simeq 2.8284$. This result, consistent with the $s_{b}=0$ numerical solution in Fig. 13, can be shown directly from the nonlinear equation (E12). However, the solution (E18) is most readily derived by solving the linear equation (E4) for $\psi$ with $K=0$ and employing Eqs. (E3) and (E8). Note that Eq. (E18) is consistent with the first two terms of the expansion in Eq. (E16) with $s_{b}=0$, showing that the series expansion truncates in this limit.

The $x$-plane kinetic temperature $T_{x}=\left\langle x^{\prime 2}\right\rangle_{\mathbf{x}_{\perp}^{\prime}}$ of the parabolic equilibrium can be calculated from Eq. (38) and previous results. This gives

$$
T_{x}(r)= \begin{cases}\frac{1}{3}\left[H_{b}-\psi(r)\right], & \psi<H_{b} \\ 0, & \psi>H_{b}\end{cases}
$$

Equation (E7) can be applied to express this result in terms of the beam density $n$ (in normalized and unnormalized form) as

$$
T_{x}=\frac{1}{3} \sqrt{\frac{n}{\pi f_{0}}}=\frac{1}{3} \sqrt{\frac{\hat{n}}{\pi f_{0}}} \sqrt{N} .
$$

This result, illustrating that $T_{x} \propto \sqrt{n}$, is a consequence of the parabolic equilibrium choice for $f_{\perp}\left(H_{\perp}\right)$. Equation (E20) can then be applied in Eq. (43) to explicitly calculate the parabolic distribution rms-edge emittance $\varepsilon_{b}=4 \sqrt{\left\langle x^{2}\right\rangle_{\perp}\left\langle x^{\prime 2}\right\rangle_{\perp}}$ in terms of the density (in normalized and unnormalized form) as

$$
\varepsilon_{b}^{2}=\frac{2}{3} \frac{r_{b}^{2}}{\sqrt{\pi f_{0}}} \frac{\int_{0}^{\infty} d r r n^{3 / 2}}{\int_{0}^{\infty} d r r n}=\frac{2}{3} \sqrt{\frac{\hat{n}}{\pi f_{0}}} r_{b}^{2} \frac{\int_{0}^{\rho_{e}} d \rho \rho N^{3 / 2}}{\int_{0}^{\rho_{e}} d \rho \rho N} .
$$

Alternatively, the emittance $\varepsilon_{b}$ can be calculated from other equilibrium parameters using the matched envelope equation (39).

It is useful to employ $H_{\perp}=\frac{1}{2} \mathbf{x}_{\perp}^{\prime 2}+\psi$ [see Eq. (35)] and Eq. (E7) to express the parabolic equilibrium distribution (E1) in the form

$$
f_{\perp}\left(\mathbf{x}_{\perp}, \mathbf{x}_{\perp}^{\prime}\right)=f_{0}\left(-\frac{1}{2} \mathbf{x}_{\perp}^{\prime 2}+\sqrt{\frac{n}{\pi f_{0}}}\right) \Theta\left(-\frac{1}{2} \mathbf{x}_{\perp}^{\prime 2}+\sqrt{\frac{n}{\pi f_{0}}}\right) .
$$

Note that $H_{b}$ has been eliminated in Eq. (E22). The maximum of the parabolic distribution occurs at $f_{\perp}\left(\mathbf{x}_{\perp}=\right.$ $0, \mathbf{x}_{\perp}^{\prime}=0$ ), where

$$
f_{\perp}\left(\mathbf{x}_{\perp}=0, \mathbf{x}_{\perp}^{\prime}=0\right) \equiv \hat{f}=\sqrt{\frac{f_{0} \hat{n}}{\pi}} .
$$

Analogous to the waterbag equilibrium case discussed in Appendix D, parameters introduced in the formulation need to be related to standard parameters employed in accelerator physics. To do this, we first calculate the beam perveance $Q$ in terms of the normalized density $N(\rho)$ to be

$$
Q=\frac{q \lambda}{2 \pi \epsilon_{0} m \gamma_{b}^{3} \beta_{b}^{2} c^{2}}=2 s_{b} k_{\beta 0}^{2} r_{s}^{2} \int_{0}^{\rho_{e}} d \rho \rho N .
$$

Here, we have employed Eq. (E8) to scale the radial coordinate and density and Eq. (E11) to simplify the coefficient. Next, the definition of the statistical beam edge radius $r_{b}=\sqrt{2\left\langle r^{2}\right\rangle_{\perp}}$ can be similarly applied to obtain

$$
\left(\frac{r_{b}}{r_{s}}\right)^{2}=2 \frac{\int_{0}^{\rho_{e}} d \rho \rho^{3} N}{\int_{0}^{\rho_{e}} d \rho \rho N} .
$$

Equations (E24) and (E25) then show that 


$$
\frac{Q}{k_{\beta 0}^{2} r_{b}^{2}}=s_{b} \frac{\left(\int_{0}^{\rho_{e}} d \rho \rho N\right)^{2}}{\int_{0}^{\rho_{e}} d \rho \rho^{3} N} .
$$

The matched-beam envelope equation (39) shows that

$$
\left(k_{\beta 0}^{2} \varepsilon_{b}\right)^{2}=\left(k_{\beta 0} r_{b}\right)^{4}-Q\left(k_{\beta 0} r_{b}\right)^{2},
$$

and Eq. (E26) can be rearranged to give

$$
\left(k_{\beta 0} r_{b}\right)^{2}=\frac{Q}{s_{b}} \frac{\int_{0}^{\rho_{e}} d \rho \rho^{3} N}{\left(\int_{0}^{\rho_{e}} d \rho \rho N\right)^{2}} .
$$

Equations (E27) and (E28) then show that

$$
\left(\frac{\varepsilon_{b}}{r_{b}}\right)^{2}=Q\left(\frac{1}{s_{b}} \frac{\int_{0}^{\rho_{e}} d \rho \rho^{3} N}{\left(\int_{0}^{\rho_{e}} d \rho \rho N\right)^{2}}-1\right)
$$

which can be employed with Eq. (E21) to identify $\sqrt{\hat{n} /\left(\pi f_{0}\right)}$, a factor useful in setting the distribution scale [see Eq. (E23)], as

$$
\sqrt{\frac{\hat{n}}{\pi f_{0}}}=Q\left(\frac{1}{s_{b}} \frac{\int_{0}^{\rho_{e}} d \rho \rho^{3} N}{\left(\int_{0}^{\rho_{e}} d \rho \rho N\right)^{2}}-1\right) \frac{\int_{0}^{\rho_{e}} d \rho \rho N}{\int_{0}^{\rho_{e}} d \rho \rho N^{3 / 2}} .
$$

Note that the integrals in Eqs. (E24)-(E30) are pure functions of the dimensionless self-field parameter $s_{b}$. Because $Q /\left(k_{\beta 0}^{2} r_{b}^{2}\right)$ is a dimensionless function of accelerator parameters, Eq. (E26) can be applied to numerically solve for $s_{b}$, or alternatively $p=-\log \left(1-s_{b}\right)$, in terms of accelerator parameters.

The rms-equivalent beam measure of relative spacecharge strength $\sigma / \sigma_{0}=\sqrt{1-Q /\left(k_{\beta 0}^{2} r_{b}^{2}\right)}$ [see Eq. (47)] can be applied with Eq. (E26) to numerically calculate the parameters $p$ and/or $s_{b}=1-e^{-p}$ as a function of $\sigma / \sigma_{0}$. This result is shown in Fig. 14 over a broad range of relative space-charge strength. Note that small values of $\sigma / \sigma_{0}$ correspond to values of self-field parameter $s_{b} e x$ tremely close of $s_{b}=1$, demonstrating that $s_{b}$ is inconvenient to describe parabolic equilibria with high space-

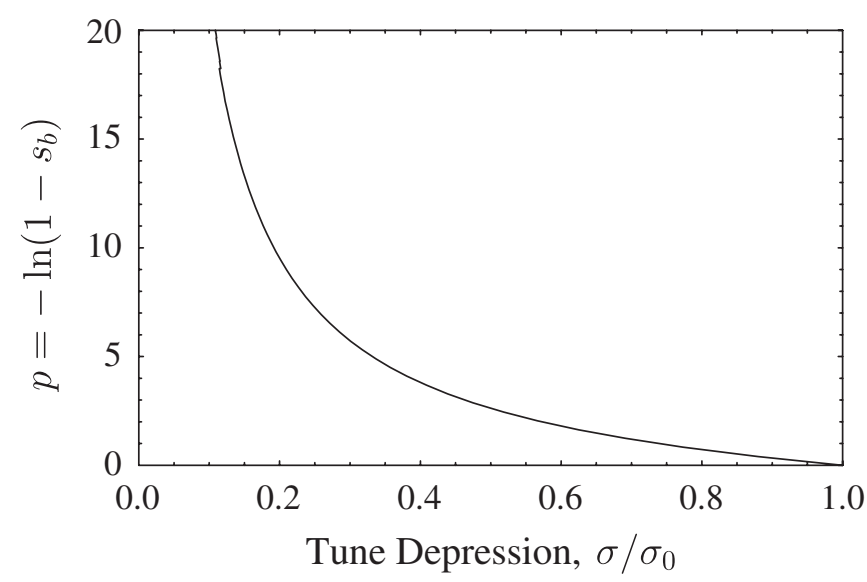

FIG. 14. Parabolic equilibrium parameter $p=-\log \left(1-s_{b}\right)$ versus rms-equivalent beam tune depression $\sigma / \sigma_{0}$ as calculated from Eqs. (E26) and (47). charge intensity. As expected, $p=0\left(s_{b}=0\right)$ corresponds to $\sigma / \sigma_{0}=1$ and a warm equilibrium with the appliedfocusing force dominating, whereas $p \rightarrow \infty\left(s_{b} \rightarrow 1\right)$ corresponds to $\sigma / \sigma_{0} \rightarrow 0$ and a cold, fully space-charge depressed equilibrium.

To better understand properties of the parabolic equilibrium, we employ Eqs. (E25) and (E26) to plot the radial density and temperature profiles and the phase-space distribution in Figs. 15 and 16 for fixed applied-focusing strength $\left(k_{\beta 0}^{2}=\right.$ const $)$ and fixed beam perveance $(Q=$ const) as the relative space-charge strength $\left(\sigma / \sigma_{0}\right)$ is varied. In Fig. 15(a) the scaled radial density profiles illustrate the sharpening of the parabolic equilibrium density profile with increasing relative space-charge strength (i.e., small $\sigma / \sigma_{0}$ or $s_{b}$ close to unity) and bell shaped for weak relative space-charge strength [i.e., $\sigma / \sigma_{0} \sim 1$, or equivalently, small $s_{b}$, with the density profile approximated by Eq. (E18)]. Similarly, the radial temperature
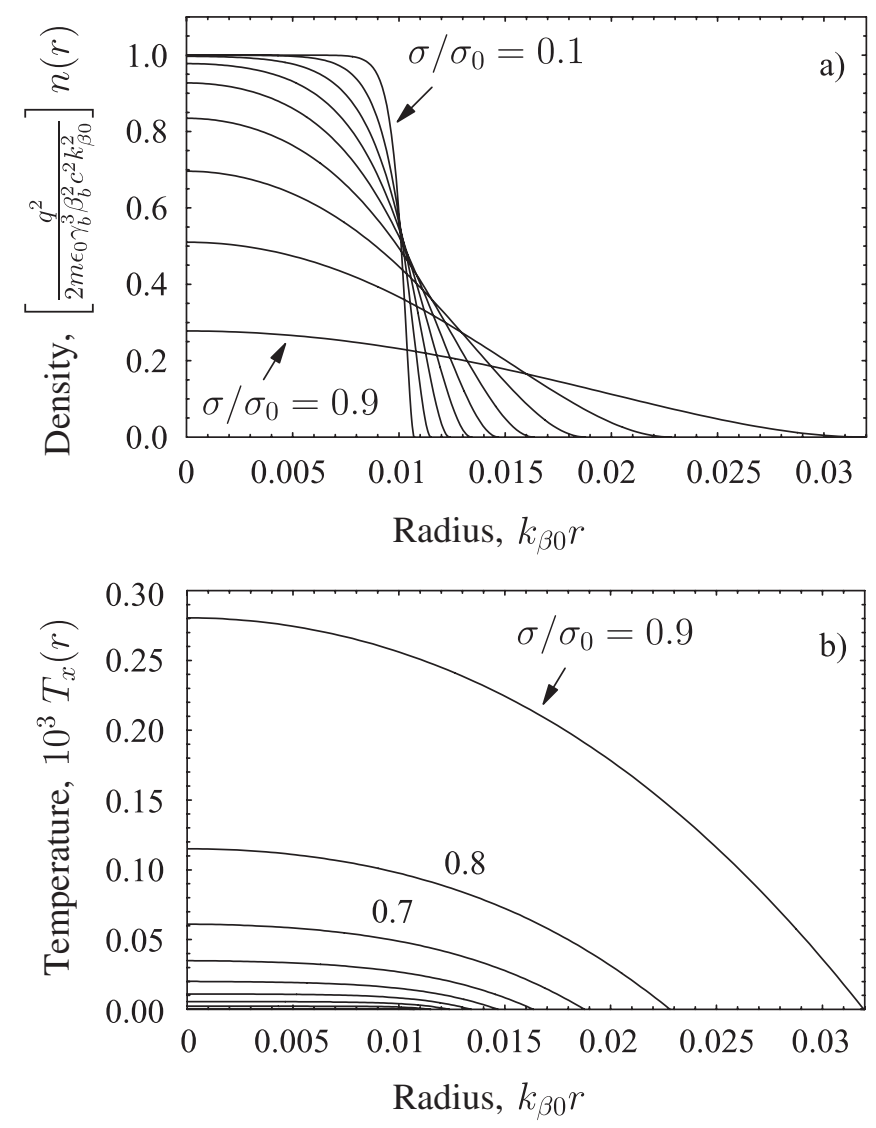

FIG. 15. Continuous-focusing parabolic equilibrium radial density and temperature profiles for fixed focusing-field strength ( $k_{\beta 0}^{2}=$ const) and perveance $Q=10^{-4}$ with (rms-equivalent beam measure) relative space-charge strengths $\sigma / \sigma_{0}=$ $0.9,0.8, \ldots, 0.1$. In (a) and (b), the scaled density $\left[q^{2} /\left(2 \epsilon_{0} m \gamma_{b}^{3} \beta_{b}^{2} c^{2} k_{\beta 0}^{2}\right)\right] n(r)$ and temperature $T_{x}(r)$ profiles are plotted versus the dimensionless radial coordinate $k_{\beta 0} r$. Values of $\sigma / \sigma_{0}$ correspond to the dimensionless equilibrium parameters in Table IV. 

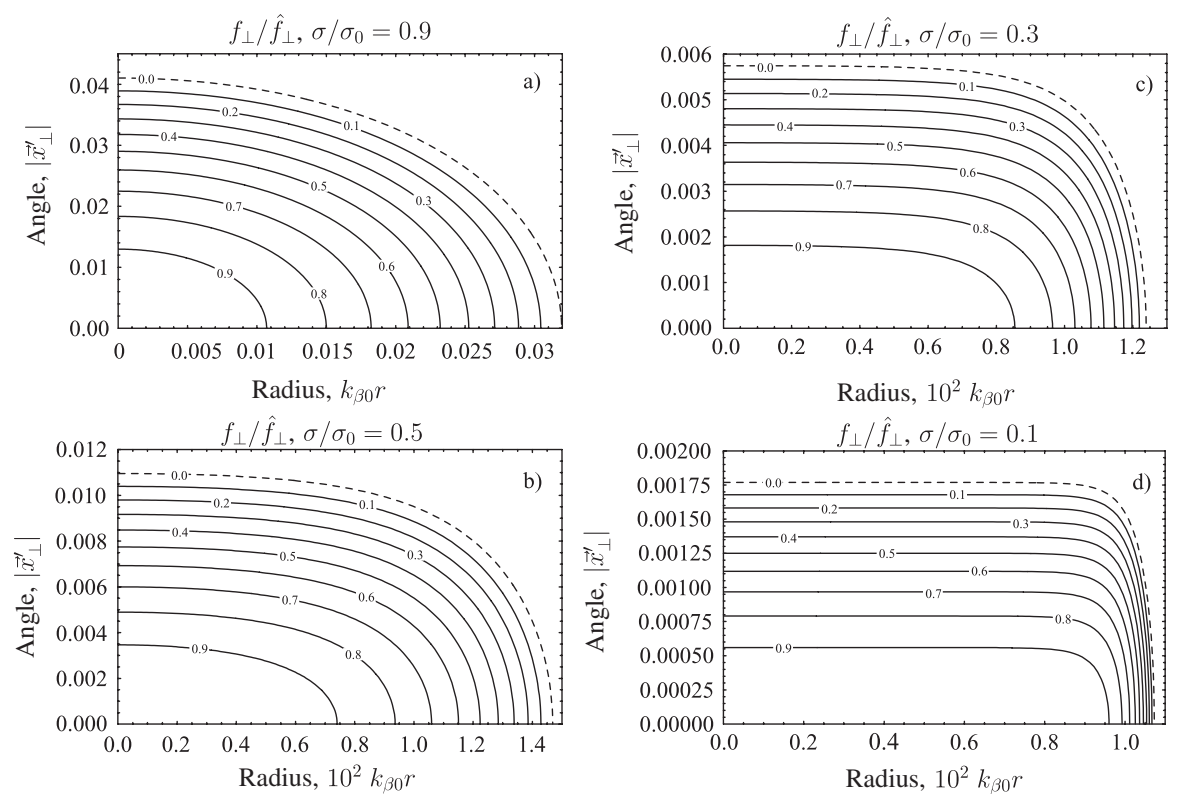

FIG. 16. Parabolic equilibrium distribution contours $f_{\perp}\left(H_{\perp}\right) / \hat{f}_{\perp}$ are plotted as a function of $k_{\beta 0} r$ and $\left|\mathbf{x}_{\perp}^{\prime}\right|$ for the profiles shown in Fig. 15 with $\sigma / \sigma_{0}=0.9,0.5,0.3$, and 0.1 in (a)-(d). Contours are labeled with the value of $f_{\perp}\left(H_{\perp}\right) / \hat{f}_{\perp}$, and the edge contour $\left(f_{\perp}=0\right)$ is represented by the dashed curve.

profile in Fig. 15(b) indicates for strong relative spacecharge forces that the temperature strongly decreases and flattens in the core of the beam before rapidly dropping to zero at the beam edge. Contours of the scaled distribution $f_{\perp}\left(H_{\perp}\right) / \hat{f}_{\perp}$ are shown in Figs. 16(a)-16(d) for values of $\sigma / \sigma_{0}$ corresponding to weak, intermediate, and strong relative space-charge strengths. The contours are generated by scaling Eq. (E22) to obtain

$$
\frac{f_{\perp}\left(H_{\perp}\right)}{\hat{f}_{\perp}}=\left(-\frac{\mathbf{x}_{\perp}^{\prime 2}}{2 \sqrt{\frac{\hat{n}}{\pi f_{0}}}}+\sqrt{N}\right) \Theta\left(-\frac{\mathbf{x}_{\perp}^{\prime 2}}{2 \sqrt{\frac{\hat{n}}{\pi f_{0}}}}+\sqrt{N}\right),
$$

and employing Eq. (E30) to calculate $\sqrt{\hat{n} /\left(\pi f_{0}\right)}$. Specific contours with $f_{\perp} / \hat{f}_{\perp} \equiv f \in[0,1]$ are then generated by plotting

$$
\left|\mathbf{x}_{\perp}^{\prime}\right|=\sqrt{2 \sqrt{\frac{\hat{n}}{\pi f_{0}}}(\sqrt{N}-f)}
$$

as a function of $\rho=r / r_{s}=\left(k_{\beta 0} r\right)\left(r_{b} / r_{s}\right) /\left(k_{\beta 0} r_{b}\right)$ for $\rho \in$ $\left[0, \rho_{f}\right]$, where $\rho_{f}$ is the numerical solution of $N\left(\rho_{f}\right)=f^{2}$. For large relative space-charge intensity $\left(\sigma / \sigma_{0} \ll 1\right)$, the flatness of the contours deep within the core of the distribution indicates nearly force-free motion until the particle enters the nonlinear edge region. Various parameters for the equilibria presented in Figs. 15 and 16 are given in Table IV.

To load the parabolic equilibrium distribution in either direct-Vlasov or PIC simulations, the general framework

TABLE IV. Dimensionless parabolic equilibrium parameters in Figs. 15 and 19 calculated for specified $\sigma / \sigma_{0}$. The values of $k_{\beta 0} \varepsilon_{b}$ are evaluated for $Q=10^{-4}$, and all other quantities are independent of $Q$.

\begin{tabular}{l|ccccc|cc}
\hline \hline & & & & & & \multicolumn{2}{c}{$Q=10^{-4}$} \\
$\sigma / \sigma_{0}$ & $p$ & $s_{b}$ & $\frac{Q}{k_{\beta 0}^{2} r_{b}^{2}}$ & $\rho_{e}$ & $\frac{r_{s}}{r_{b}}$ & $10^{3} \times k_{\beta 0} r_{b}$ & $10^{3} \times k_{\beta 0} \varepsilon_{b}$ \\
\hline 0.9 & 0.3254 & 0.2778 & 0.19 & 3.115 & 0.4471 & 22.94 & 0.4737 \\
0.8 & 0.7137 & 0.5102 & 0.36 & 3.471 & 0.3942 & 16.67 & 0.2222 \\
0.7 & 1.191 & 0.6960 & 0.51 & 3.925 & 0.3415 & 14.00 & 0.1373 \\
0.6 & 1.800 & 0.8347 & 0.64 & 4.526 & 0.2891 & 12.50 & 0.09375 \\
0.5 & 2.619 & 0.9271 & 0.75 & 5.360 & 0.2373 & 11.55 & 0.06667 \\
0.4 & 3.805 & 0.9778 & 0.84 & 6.598 & 0.1864 & 10.91 & 0.04762 \\
0.3 & 5.730 & 0.9968 & 0.91 & 8.626 & 0.1370 & 10.48 & 0.03297 \\
0.2 & 9.520 & 0.99993 & 0.96 & 12.59 & 0.08955 & 10.21 & 0.02083 \\
0.1 & 21.87 & 0.9999999997 & 0.99 & 24.27 & 0.04398 & 10.05 & 0.01010 \\
\hline \hline
\end{tabular}


presented in Sec. III B can be applied. For PIC loading of the parabolic distribution, the radial probability transform (51) for loading macroparticle coordinates $\mathbf{x}_{\perp}$ can be expressed in scaled form as

$$
\frac{\int_{0}^{\rho} d \tilde{\rho} \tilde{\rho} N(\tilde{\rho})}{\int_{0}^{\rho_{e}} d \rho \rho N(\rho)}=\hat{u}_{\rho},
$$

where $\hat{u}_{\rho} \in[0,1)$ is an independent, uniformly distributed random number generated for each macroparticle. Equation (E33) is solved for $\rho\left(\hat{u}_{\rho}\right) \in\left[0, \rho_{e}\right)$, and macroparticle coordinates $\mathbf{x}_{\perp}$ are set using $r=r_{s} \rho$ in Eq. (52). Values can be saved on a radial grid to efficiently load many particles with the same equilibrium parameters. To load the macroparticle angles $\mathbf{x}_{\perp}^{\prime}$ with the macroparticle coordinates $\mathbf{x}_{\perp}$ already loaded, the probability transform (53) is applied with Eq. (E31). Carrying out the integrals leads to a quadratic equation that can be solved for the smallest physical solution as

$$
U\left(\hat{u}_{U}\right)=\sqrt{\frac{\hat{n}}{\pi f_{0}}} \sqrt{N}\left[1-\sqrt{1-\hat{u}_{U}}\right] .
$$

Here, $\hat{u}_{U} \in[0,1)$ is an independent, uniformly distributed random number generated for each macroparticle, $\sqrt{\hat{n} /\left(\pi f_{0}\right)}$ is calculated (once) for the equilibrium parameters using Eq. (E30), and $N=N(\rho)$ is the density at the loaded radial macroparticle coordinate $\rho=\sqrt{\mathbf{x}_{\perp}^{2}} / r_{s}$. Macroparticle angles are set using this value of $U\left(\hat{u}_{U}\right)$ in Eq. (54). To efficiently carry out this angle loading procedure using Eq. (E34), $N$ should be calculated once on a grid for $\rho \in\left[0, \rho_{e}\right]$ and gridded values can then be interpolated for more accuracy.

\section{APPENDIX F: CONTINUOUS-FOCUSING THERMAL EQUILIBRIUM DISTRIBUTION}

The thermal equilibrium distribution has been studied extensively in nonneutral plasma physics $[30,110]$ and in accelerator physics by Reiser [31,111] and others $[32,67,68,112,113]$. Here we review previous results in a format that allows easy contrast to other continuousfocusing distributions (see Appendices D and E) while presenting extensions needed for practical implementation of Vlasov simulation loads using standard inputs for accelerator physics. For a thermal equilibrium distribution in continuous focusing, we take

$$
f_{\perp}\left(H_{\perp}\right)=\frac{m \gamma_{b} \beta_{b}^{2} c^{2} \hat{n}}{2 \pi T} \exp \left(-\frac{m \gamma_{b} \beta_{b}^{2} c^{2} H_{\perp}}{T}\right),
$$

where $\hat{n}=$ const is a constant density scale, and $T=$ const is the thermodynamic temperature (expressed in energy units) in the laboratory frame. The thermal equilibrium distribution is a special class of stable Vlasov equilibria with $\partial f_{\perp} / \partial H_{\perp} \leq 0$ [30]. Within the weak coupling approximation $\left(q^{2} / \hat{n}^{-2 / 3} \ll T\right)$, any initial distribution func- tion $f_{\perp}\left(\mathbf{x}_{\perp}, \mathbf{x}_{\perp}^{\prime}, s=s_{i}\right)$, however complex, relaxes through collisions to the thermal equilibrium form in Eq. (F1). This is true regardless of the details of the intervening evolution due to both collective and collisional processes. Even stable Vlasov equilibria must ultimately relax to thermal equilibrium form due to collisional effects outside the Vlasov model. Although the time scales for collisional relaxation are usually long relative to beam residence times in a machine, couplings to external error sources together with collective effects can result in enhanced rates of effective thermalization. In this regard, thermal equilibrium can be regarded as a preferred equilibrium state of the system.

The thermal equilibrium distribution is characterized by a radial kinetic temperature profile that is uniform. Direct calculation with Eqs. (14) and (F1) shows that

$$
\begin{aligned}
T_{x}=T_{y} & =\frac{\int d^{2} x_{\perp}^{\prime} x^{\prime 2} f_{\perp}}{\int d^{2} x_{\perp}^{\prime} f_{\perp}} \\
& =\frac{T}{m \gamma_{b} \beta_{b}^{2} c^{2}} \equiv T^{*}=\text { const. }
\end{aligned}
$$

This constant temperature results in a diffuse beam edge since the spread in particle transverse energy will prevent an abrupt turning point of all particles. For analysis of the radial density profile and the Poisson equation of the equilibrium, we employ the formulation developed in Sec. III B. For the thermal equilibrium distribution it is convenient to define a dimensionless potential

$$
\tilde{\psi}(r)=\frac{\psi(r)}{T^{*}}=\frac{1}{T}\left[\frac{m \gamma_{b} \beta_{b}^{2} c^{2} k_{\beta 0}^{2} r^{2}}{2}+\frac{q \phi(r)}{\gamma_{b}^{2}}\right],
$$

and make, without loss of generality, the choice of potential reference $\phi(r=0)=0$. Then Eqs. (36) and (F1) can be employed to calculate the equilibrium radial density profile in terms of $\tilde{\psi}$. This gives

$$
n(r)=\int d^{2} x_{\perp}^{\prime} f_{\perp}\left(H_{\perp}\right)=\hat{n} e^{-\tilde{\psi}(r)} .
$$

Because $\tilde{\psi}(r=0)=0, \hat{n}$ is identified as the on-axis density of the equilibrium. Using Eq. (F4), the transformed Poisson equation (37) can be recast in scaled form as

$$
\frac{1}{\rho} \frac{\partial}{\partial \rho}\left(\rho \frac{\partial \tilde{\psi}}{\partial \rho}\right)=1+\Delta-e^{-\tilde{\psi}},
$$

and solved subject to the boundary conditions $\tilde{\psi}(\rho=0)=$ 0 and $\left.\frac{\partial \tilde{\psi}}{\partial \rho}\right|_{\rho=0}=0$. Here, $\rho \equiv r /\left(\gamma_{b} \lambda_{D}\right)$ is a scaled radial coordinate with $\lambda_{D} \equiv\left[T /\left(m \hat{\omega}_{p}^{2}\right)\right]^{1 / 2}$ and $\hat{\omega}_{p} \equiv$ $\left[q^{2} \hat{n} /\left(\epsilon_{0} m\right)\right]^{1 / 2}$ denoting the Debye length and plasma frequency formed from the (on-axis) density scale $\hat{n}$, and $\Delta$ is defined by

$$
\Delta \equiv \frac{2 \gamma_{b}^{3} \beta_{b}^{2} c^{2} k_{\beta 0}^{2}}{\hat{\omega}_{p}^{2}}-1
$$




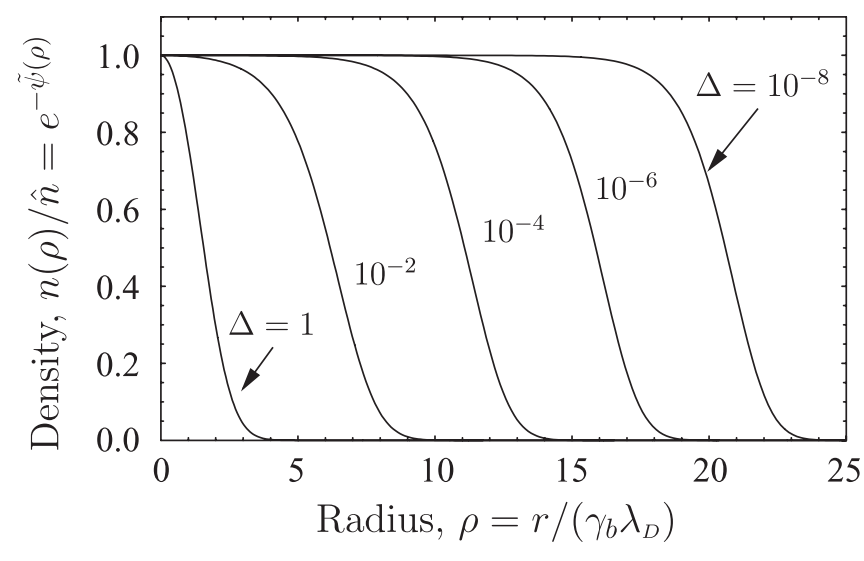

FIG. 17. For a thermal equilibrium, the scaled density $n(\rho) / \hat{n}=\exp (-\tilde{\psi})$ is plotted versus the scaled radial coordinate $\rho=r /\left(\gamma_{b} \lambda_{D}\right)$ calculated from the solution of the scaled thermal equilibrium Poisson equation (F5) for $\Delta=10^{-\ell}$ with $\ell=0,2$, 4,6 , and, 8 .

Here, $\Delta \in(0, \infty)$ is a positive, dimensionless parameter relating the ratio of applied to space-charge defocusing forces. Note that $\Delta$ is simply a convenient rescaling of the self-field parameter $s_{b} \equiv \hat{\omega}_{p}^{2} /\left(2 \gamma_{b}^{3} \beta_{b}^{2} c^{2} k_{\beta 0}^{2}\right)$ defined in Eq. (48) with

$$
\Delta=\frac{1}{s_{b}}-1
$$

Strictly speaking, from the form of the thermal equilibrium density profile in Eq. (F4), it follows that the radial density profile $n(r)$ is nonzero for any finite radius $r<\infty$ and the thermal equilibrium distribution is only consistent with a free-space model with no conducting beam pipe $\left(r_{p} \rightarrow \infty\right)$. However, since the density becomes exponentially small at large radii, this nonzero density inconsistency can be ignored if the cylindrical pipe radius $r_{p}$ is chosen sufficiently large in comparison with the rms-envelope radius $r_{b}$. Here we analyze properties of thermal equilibrium beams in the formally correct, infinite-system limit. Modified thermal-equilibrium-like distributions have also been presented that modify Eq. (F1) to introduce a sharp cutoff [114]. In some applications, this can improve the model at the expense of introducing another parameter to identify.

It is useful to employ $H_{\perp}=\frac{1}{2} \mathbf{x}_{\perp}^{\prime 2}+\psi$ [see Eq. (35)] and Eqs. (F3) and (F4) to express the thermal equilibrium distribution (F1) as

$$
f_{\perp}\left(\mathbf{x}_{\perp}, \mathbf{x}_{\perp}^{\prime}\right)=\hat{n} e^{-\left[\mathbf{x}_{\perp}^{\prime 2} /\left(2 T^{*}\right)\right]} e^{-\tilde{\psi}}=n(r) e^{-\left[\mathbf{x}_{\perp}^{\prime 2} /\left(2 T^{*}\right)\right]} .
$$

The maximum of the thermal distribution occurs at $\mathbf{x}_{\perp}=0$ and $\mathbf{x}_{\perp}^{\prime}=0$, where $f_{\perp}\left(\mathbf{x}_{\perp}=0, \mathbf{x}_{\perp}^{\prime}=0\right)=\hat{n}$.

The thermal equilibrium distribution parameters corresponding to the on-axis density $\hat{n}$, the thermodynamic temperature $T$, and the parameter $\Delta$ must be related to standard quantities associated with accelerator physics as discussed in Sec. III B. To carry out this procedure, the transformed Poisson equation (F5) is first solved for $\tilde{\psi}$ to obtain the thermal equilibrium density profile from Eq. (F4) and calculate the needed parametric constraints. This equation is highly nonlinear and must, in general, be solved numerically [91,115]. However, closed-form approximate analytical solutions have recently been constructed for both large and small values of $\Delta$ that are highly accurate [116]. The numerical solution is illustrated in Fig. 17, where the normalized density $n(\rho) / \hat{n}=$ $\exp (-\tilde{\psi})$ is plotted versus $\rho=r /\left(\gamma_{b} \lambda_{D}\right)$ for values of $\Delta$ covering several decades. Note that for small values of $\Delta$, the scaled density $n(r) / \hat{n}$ varies little from unity from $\rho=$ 0 until intermediate-to-large values of $\rho$ [corresponding to a large number of Debye lengths, since $\rho=r /\left(\gamma_{b} \lambda_{D}\right)$ ], where the density profile rapidly falls to exponentially small values as $\rho$ increases by $4-5$ units (i.e., Debye lengths). Note that the width in $\rho$ of the radial falloff varies little with $\Delta$, whereas the width of the flat, central region is a strong function of $\Delta$. It will be shown that this highly nonlinear regime of small $\Delta$, with $\Delta \sim 10^{-6}$ and smaller can correspond to beam parameters of practical interest when the space-charge intensity is strong and the beam can be many Debye lengths in radial extent. In this regime, conventional numerical methods to integrate Eq. (F5) for $\tilde{\psi}$ as a function of $\rho$ from the on-axis values $\tilde{\psi}(\rho=0)=0$ and $\partial \tilde{\psi} /\left.\partial \rho\right|_{\rho=0}=0$ can fail. This parametric sensitivity is evident from the extreme flatness of $n(\rho)$ for $\rho \ll 1$ and small $\Delta$. Small, high-order derivative values of $\tilde{\psi}$ at $\rho=0$ sensitively determine the value of $\rho$ where the rapid edge falloff begins for $\Delta$ small, complicating numerical solutions. We address this issue in Appendix G, where an analytical series solution of the scaled Poisson equation (F5) is developed that is valid within the core of the beam. Use of this series solution allows the integration to be initiated at a value of $\rho>0$ where there is sufficient variation that standard numerical methods can be applied to generate solutions for $\tilde{\psi}(\rho)$ for arbitrarily small values of $\Delta$. In the emittance-dominated regime, $\Delta \gg 1$, and the solution to the scaled Poisson equation becomes $\tilde{\psi} \simeq(1+$ $\Delta) \rho^{2} / 4$, and the scaled density profile $n=\hat{n} \exp (-\tilde{\psi})$ becomes Gaussian in $r$ with

$$
n(r) \simeq \hat{n} \exp \left[-\frac{(1+\Delta)}{4}\left(\frac{r}{\gamma_{b} \lambda_{D}}\right)^{2}\right] .
$$

Note from Fig. 17 that $n(r)$ is well approximated by Eq. (F9) even when $\Delta \sim 1$, showing that even modest values of $\Delta$ correspond to weak space-charge intensity. For $\Delta \ll 1$ and arbitrarily small but nonzero, a nonlinear analysis presented in Ref. [116] shows that $n(r)$ is well approximated by

$$
n(r) \simeq \hat{n} \frac{\left(1+\frac{1}{2} \Delta+\frac{1}{24} \Delta^{2}\right)^{2}}{\left\{1+\frac{1}{2} \Delta I_{0}\left(\frac{r}{\gamma_{b} \lambda_{D}}\right)+\frac{1}{24}\left[\Delta I_{0}\left(\frac{r}{\gamma_{b} \lambda_{D}}\right)\right]^{2}\right\}^{2}},
$$

where $I_{0}(x)$ denotes an order zero modified Bessel function 
[108]. The closed-form solution given by Eq. (F10) is highly accurate outside of the far tail where $N<0.001$ when $\Delta<10^{-2}$.

Specification of the line-charge density $\lambda$ [see Eq. (10)] and the transverse energy of the beam macrostate fixes the values of the constants $\hat{n}$ and $T$. Alternatively, we derive constraints to relate the thermal equilibrium parameters $\hat{n}$, $T$, and $\Delta$, or equivalently, the effective Debye length $\gamma_{b} \lambda_{D}=\gamma_{b} \sqrt{\epsilon_{0} T /\left(q^{2} \hat{n}\right)}$, the scaled temperature $T^{*}=$ $T /\left(m \gamma_{b} \beta_{b}^{2} c^{2}\right)$, and $\Delta$, in terms of the focusing strength $k_{\beta 0}$, the perveance $Q$ [see Eq. (22)], and the emittance $\varepsilon_{b}$ [see Eq. (42)] using the formulation developed in Sec. III B. First, the beam line-charge density $\lambda=$ $2 \pi q \int_{0}^{\infty} d r r n(r)$ and the beam rms-edge radius $r_{b}=$ $\sqrt{2\left\langle r^{2}\right\rangle_{\perp}}$ are expressed in terms of the thermal equilibrium density in Eq. (F4) as

$$
\begin{aligned}
\lambda & =\frac{\gamma_{b}^{2} T}{2 q} \int_{0}^{\infty} d \rho \rho e^{-\tilde{\psi}}, \\
r_{b}^{2} & =2 \gamma_{b}^{2} \lambda_{D}^{2} \frac{\int_{0}^{\infty} d \rho \rho^{3} e^{-\tilde{\psi}}}{\int_{0}^{\infty} d \rho \rho e^{-\tilde{\psi}}} .
\end{aligned}
$$

Note that the integrals occurring in Eq. (F11) depend only on the parameter $\Delta$ with the solution $\tilde{\psi}(\rho)$ formally given by the scaled Poisson equation (F5). Next, the rms-edge emittance $\varepsilon_{b}$ is simply calculated directly from $\varepsilon_{b}^{2}=$ $2 r_{b}^{2}\left\langle\mathbf{x}_{\perp}^{\prime 2}\right\rangle_{\perp}$ and the thermal equilibrium distribution function (F1) to show that

$$
\varepsilon_{b}^{2}=4 T^{*} r_{b}^{2}
$$

The matched-beam envelope equation (39) and $\varepsilon_{b}^{2}$ in Eq. (F12) can be used to express equivalently the rmsenvelope radius $r_{b}$ as

$$
r_{b}^{2}=\frac{1}{k_{\beta 0}^{2}}\left(4 T^{*}+Q\right) .
$$

Equation (F11) and the definition of the perveance $Q=$ $2 q \lambda /\left(2 \pi \epsilon_{0} m \gamma_{b}^{3} \beta_{b}^{2} c^{2}\right)$ obtains the constraint

$$
Q=T^{*} \int_{0}^{\infty} d \rho \rho e^{-\tilde{\psi}}
$$

and Eqs. (F11) and (F12) can be combined to yield the constraint

$$
k_{\beta 0}^{2} \varepsilon_{b}^{2}=4 T^{*}\left(4 T^{*}+Q\right)
$$

Then Eq. (F6) and the Debye length definition $\lambda_{D}=$ $\left(T / \hat{\omega}_{p}\right)^{1 / 2}$ yield

$$
k_{\beta 0}^{2}=T^{*} \frac{(1+\Delta)}{2 \gamma_{b}^{2} \lambda_{D}^{2}} .
$$

Equation (F15) can be solved analytically for $T^{*}$, and the constraints in Eqs. (F14)-(F16) expressed as

$$
\begin{aligned}
T^{*} & =\frac{Q}{8}\left(\sqrt{1+4 k_{\beta 0}^{2} \varepsilon_{b}^{2} / Q^{2}}-1\right), \\
8 & =\left(\sqrt{1+4 k_{\beta 0}^{2} \varepsilon_{b}^{2} / Q^{2}}-1\right) \int_{0}^{\infty} d \rho \rho e^{-\tilde{\psi}} \\
\left(k_{\beta 0} \gamma_{b} \lambda_{D}\right)^{2} & =\frac{Q}{16}(1+\Delta)\left(\sqrt{1+4 k_{\beta 0}^{2} \varepsilon_{b}^{2} / Q^{2}}-1\right) .
\end{aligned}
$$

The constraint equations (F17) provide relations fixing $\gamma_{b} \lambda_{D}, T^{*}=T /\left(m \gamma_{b} \beta_{b}^{2} c^{2}\right)$, and $\Delta$ in terms of $Q, \varepsilon_{b}$, and $k_{\beta 0}$. Note that the integral $\int_{0}^{\infty} d \rho \rho \exp (-\tilde{\psi})$ is an implicit function of $\Delta$ and must, in general, be calculated numerically to fully solve the constraints. In some applications it is useful to explicitly identify the on-axis density scale $n(r=0)=\hat{n}$ in terms of accelerator parameters. This can be done by rewriting Eq. (F16) as

$$
\hat{n}=\frac{2 \epsilon_{0} m \gamma_{b}^{3} \beta_{b}^{2} c^{2} k_{\beta 0}^{2}}{(1+\Delta) q^{2}} .
$$

We first employ Eqs. (F11) and (F17) to reinforce the interpretation that the $\Delta$ can be regarded as a parameter related to the relative space-charge strength. Using these constraints, the rms-equivalent beam tune depression $\sigma / \sigma_{0} \equiv \sqrt{1-Q /\left(k_{\beta 0}^{2} r_{b}^{2}\right)}$ [see Eq. (47)] and $\Delta$ can be related by

$$
\frac{\sigma}{\sigma_{0}}=\left\{1-\frac{\left[\int_{0}^{\infty} d \rho \rho e^{-\tilde{\psi}}\right]^{2}}{(1+\Delta) \int_{0}^{\infty} d \rho \rho^{3} e^{-\tilde{\psi}}}\right\}^{1 / 2} .
$$

This equation is solved numerically to plot $\Delta$ as a function of $\sigma / \sigma_{0}$ in Fig. 18. Note that strong tune depressions with $\sigma / \sigma_{0}<0.2$ correspond to extremely small values of $\Delta$. Because $\Delta$ is a single-valued function of $\sigma / \sigma_{0}$, the relative space-charge strength uniquely determines $\Delta$. Al-

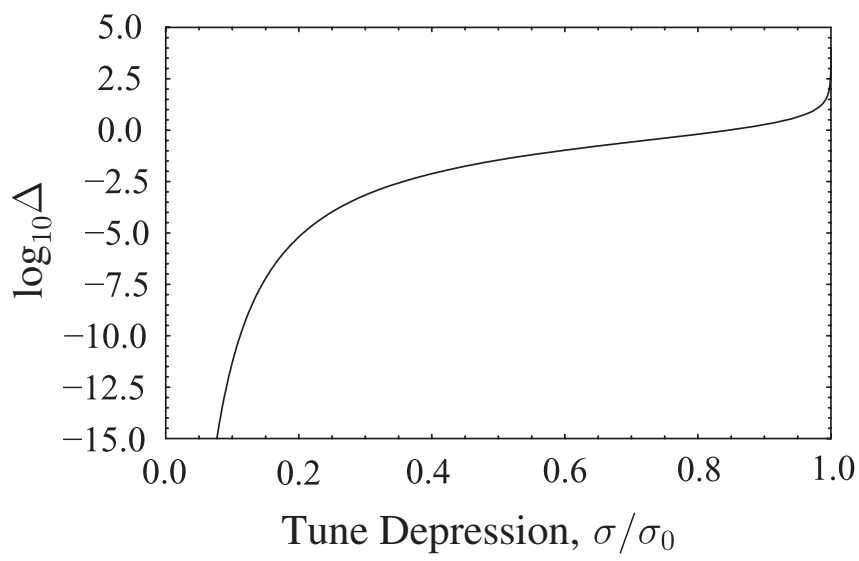

FIG. 18. Thermal equilibrium parameter $\Delta$ plotted versus rmsequivalent beam tune depression $\sigma / \sigma_{0}$ as calculated from Eq. (F19). 

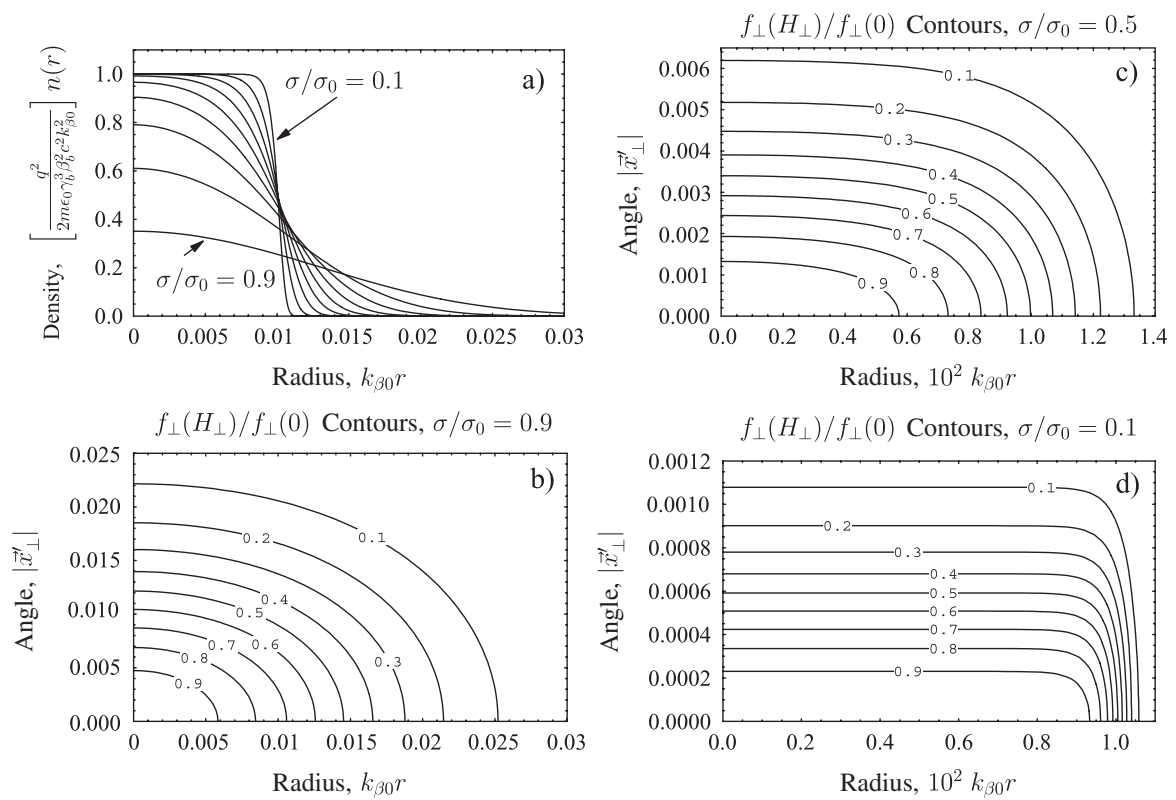

FIG. 19. Thermal equilibrium distribution in continuous-focusing field for fixed focusing strength $\left(k_{\beta 0}^{2}=\right.$ const) and perveance $Q=$ $10^{-4}$ (rms-equivalent beam measure). In (a) the scaled density $\left[q^{2} /\left(2 \epsilon_{0} m \gamma_{b}^{3} \beta_{b}^{2} c^{2} k_{\beta 0}^{2}\right)\right] n(r)$ is plotted versus the dimensionless radial coordinate $k_{\beta 0} r$ for relative space-charge strengths $\sigma / \sigma_{0}=0.9,0.8, \ldots, 0.1$, and in (b), (c), and (d), the normalized distribution $f_{\perp}\left(H_{\perp}\right) / f_{\perp}(0)=$ const contours are plotted as a function of $k_{\beta 0} r$ and $\left|\mathbf{x}_{\perp}^{\prime}\right|$ for $\sigma / \sigma_{0}=0.9,0.5$, and 0.1. Contours are labeled by the value of $f_{\perp}\left(H_{\perp}\right) / \hat{f}_{\perp}$. Values of $\sigma / \sigma_{0}$ correspond to the dimensionless equilibrium parameters in Table $\mathrm{V}$.

ternatively, the on-axis self-field parameter $s_{b} \equiv$ $\hat{\omega}_{p}^{2} /\left(2 \gamma_{b}^{3} \beta_{b}^{2} c^{2} k_{\beta 0}^{2}\right)=1 /(1+\Delta)$ [see Eq. (F7)] can be employed in place of $\sigma / \sigma_{0}$ to specify the scaled equilibrium. The physical range of $\Delta>0$ implies that $s_{b} \in[0,1)$ for the thermal equilibrium distribution. Note that $s_{b}$ will be extremely close to unity for small $\sigma / \sigma_{0}$ corresponding to beams with high space-charge intensity intensity, rendering $s_{b}$ a less convenient parameter to describe thermal equilibria in the space-charge-dominated regime. Generally, when numerically solving for needed values of $\Delta$ for thermal equilibria with high space-charge intensity $\left(\sigma / \sigma_{0}\right.$ small $)$, it can be more convenient to use $\Delta=e^{p}$ and solve for $p$ due to the sensitivity of the equilibrium specification in $\Delta$ [or $\left.s_{b}=1 /(1+\Delta)\right]$.
To better understand properties of the thermal equilibrium, we employ Eqs. (F17) and (F18) to plot the radial density profile and the phase-space distribution in Fig. 19 for fixed applied-focusing strength $\left(k_{\beta 0}^{2}=\right.$ const $)$ and fixed beam perveance ( $Q=$ const) as the relative space-charge strength $\left(\sigma / \sigma_{0}\right)$ is varied. In Fig. 19(a) the scaled radial density is plotted. For thermal equilibrium, the temperature profile is spatially uniform with $T_{x}=T_{y}=$ $T /\left(m \gamma_{b} \beta_{b}^{2} c^{2}\right)=T^{*}=$ const [see Eq. (F2)]. Contours of the scaled distribution $f_{\perp}\left(H_{\perp}\right) / f_{\perp}(0)$ are shown in Figs. 19(b)-19(d) for values of $\sigma / \sigma_{0}$ corresponding to weak, intermediate, and strong relative space-charge intensity. Various parameters for the equilibria presented in Fig. 19 are given in Table V. Figure 19(a) illustrates how

TABLE V. Dimensionless thermal equilibrium parameters in Fig. 19 calculated for specified $\sigma / \sigma_{0}$. The values of $k_{\beta 0} \gamma_{b} \lambda_{D}, T^{*}=$ $T /\left(m \gamma_{b} \beta_{b}^{2} c^{2}\right)$, and $k_{\beta 0} \varepsilon_{b}$ are evaluated for $Q=10^{-4}$, and all other quantities are independent of $Q$.

\begin{tabular}{|c|c|c|c|c|c|c|c|c|}
\hline$\sigma / \sigma_{0}$ & $\Delta$ & $s_{b}$ & $\frac{Q}{k_{\beta 0}^{2} r_{b}^{2}}$ & $\frac{k_{\beta 0}^{2} \varepsilon_{b}^{2}}{Q^{2}}$ & $k_{\beta 0} \gamma_{b} \lambda_{D}$ & $\frac{T}{m \gamma_{b} \beta_{b}^{2} c^{2}}$ & $\begin{array}{l}10^{-4} \\
10^{3} \times k_{\beta 0} r_{b}\end{array}$ & $10^{3} \times k_{\beta 0} \varepsilon_{b}$ \\
\hline 0.9 & 1.851 & 0.3508 & 0.19 & 22.44 & 12.33 & $1.065 \times 10^{-4}$ & 22.94 & 0.4737 \\
\hline 0.8 & $6.382 \times 10^{-1}$ & 0.6104 & 0.36 & 4.938 & 6.034 & $4.444 \times 10^{-5}$ & 16.67 & 0.2222 \\
\hline 0.7 & $2.649 \times 10^{-1}$ & 0.7906 & 0.51 & 1.884 & 3.898 & $2.402 \times 10^{-5}$ & 14.00 & 0.1373 \\
\hline 0.6 & $1.059 \times 10^{-1}$ & 0.9043 & 0.64 & 0.8789 & 2.788 & $1.406 \times 10^{-5}$ & 12.50 & 0.09375 \\
\hline 0.5 & $3.501 \times 10^{-2}$ & 0.9662 & 0.75 & 0.4444 & 2.077 & $8.333 \times 10^{-6}$ & 11.55 & 0.06667 \\
\hline 0.4 & $7.684 \times 10^{-3}$ & 0.9924 & 0.84 & 0.2268 & 1.549 & $4.762 \times 10^{-6}$ & 10.91 & 0.04762 \\
\hline 0.3 & $6.950 \times 10^{-4}$ & 0.9993 & 0.91 & 0.1087 & 1.112 & $2.473 \times 10^{-6}$ & 10.48 & 0.03297 \\
\hline 0.2 & $6.389 \times 10^{-6}$ & 0.999994 & 0.96 & 0.04340 & 0.7217 & $1.042 \times 10^{-6}$ & 10.21 & 0.02083 \\
\hline 0.1 & $4.975 \times 10^{-12}$ & 0.999999999995 & 0.99 & 0.01020 & 0.3553 & $2.525 \times 10^{-7}$ & 10.05 & 0.01010 \\
\hline
\end{tabular}


the thermal equilibrium density profile sharpens and becomes more step-function-like with increasing relative space-charge strength (i.e., small $\sigma / \sigma_{0}$, or equivalently small $T^{*}$ ) and Gaussian-like for weak relative space-charge strength [i.e., $\sigma / \sigma_{0} \sim 1$, or equivalently large $T^{*}$, with the density profile approximated by Eq. (F9)]. Note that the peak density $\hat{n}$ of the beam increases with increasing space-charge strength, whereas the rms-envelope radius $r_{b}=\sqrt{2\left\langle r^{2}\right\rangle_{\perp}}$ decreases with increasing space-charge strength.

To load the thermal equilibrium distribution in either direct-Vlasov or PIC simulations, the general framework presented in Sec. III B can be applied. For PIC loading, the radial probability transform (51) for loading macroparticle coordinates $\mathbf{x}_{\perp}$ is straightforward to apply using the normalized coordinates defined by

$$
\frac{\int_{0}^{\rho} d \tilde{\rho} \rho e^{-\tilde{\psi}(\tilde{\rho})}}{\int_{0}^{\infty} d \rho \rho e^{-\tilde{\psi}(\rho)}}=\hat{u}_{\rho},
$$

where $\hat{u}_{\rho} \in[0,1)$ is a uniformly distributed random number generated for each macroparticle. Equation (F20) is solved for $\rho\left(\hat{u}_{\rho}\right)$ and macroparticle coordinates $\mathbf{x}_{\perp}$ are set using $r=\gamma_{b} \lambda_{D} \rho\left(\hat{u}_{\rho}\right)$ in Eq. (52). Values can be saved on a radial grid in $r$ out to a maximum cutoff value, where the beam density is negligible, to efficiently load many macroparticles with the same equilibrium parameters. For loading the macroparticle angles $\mathbf{x}_{\perp}^{\prime}$, the probability transform (53) can be greatly simplified for the thermal equilibrium distribution. Independent of macroparticle position, we have

$$
U\left(\hat{u}_{U}\right)=-T^{*} \log \left(1-\hat{u}_{U}\right),
$$

where $\hat{u}_{U} \in[0,1)$ is a uniformly distributed random number generated for each macroparticle. Using the constraint equation (F12) and this result shows that the angle loading given by Eq. (54) can be expressed as

$$
\begin{aligned}
& x^{\prime}=\frac{\varepsilon_{b}}{2 r_{b}} \sqrt{-2 \log \left(1-\hat{u}_{U}\right)} \cos \left(2 \pi \hat{u}_{\varphi}\right), \\
& y^{\prime}=\frac{\varepsilon_{b}}{2 r_{b}} \sqrt{-2 \log \left(1-\hat{u}_{U}\right)} \sin \left(2 \pi \hat{u}_{\varphi}\right),
\end{aligned}
$$

where $\hat{u}_{\varphi} \in[0,1)$ is a uniformly distributed random number generated for each macroparticle. Using twodimensional probability transforms [95], this result can be shown to be equivalent to

$$
x^{\prime}=\frac{\varepsilon_{b}}{2 r_{b}} \hat{g}_{x}, \quad y^{\prime}=\frac{\varepsilon_{b}}{2 r_{b}} \hat{g}_{y},
$$

where $\hat{g}_{x}$ and $\hat{g}_{y}$ are independent, Gaussian-distributed random numbers with unit variance.

\section{APPENDIX G: SERIES SOLUTION OF POISSON'S EQUATION FOR THE CONTINUOUS-FOCUSING THERMAL EQUILIBRIUM DISTRIBUTION}

The scaled thermal equilibrium Poisson equation (F5), $(1 / \rho)(\partial / \partial \rho)(\rho \partial \tilde{\psi} / \partial \rho)=1+\Delta-\exp (-\tilde{\psi})$ is most naturally numerically integrated for $\tilde{\psi}$ as a function of $\rho$ from the on-axis values $\tilde{\psi}(\rho=0)=0$ and $\partial \tilde{\psi} /\left.\partial \rho\right|_{\rho=0}=$ 0 . In regimes of practical interest corresponding to very cold beams ( $T$ small), the parameter $\Delta$ can be $\sim 10^{-6}$ and smaller. For such small values of $\Delta$, the scaled density profile $n(\rho) / \hat{n}=\exp (-\tilde{\psi})$ (see Fig. 17) is very flat for small $\rho$, and falls abruptly to zero at intermediate-to-large values of $\rho$. This highly sensitive parametric dependence on $\Delta$ renders the direct numerical integration difficult (i.e., the system is very stiff) using conventional numerical methods. For this reason, most work on thermal equilibrium beams has focused on values of $\Delta$ sufficiently high that numerical issues are easily avoided. Here, we outline a series solution for the thermal equilibrium density profile [115] valid for intermediate values of $\rho$ that can be employed to construct accurate numerical solutions over the entire range of $\rho$ for arbitrarily small values of $\Delta$, thereby enabling the analysis of arbitrarily low-temperature thermal equilibrium beams. The methods described are employed to generate the solutions needed for the explicit calculation of thermal equilibrium quantities illustrated in Appendix F.

Operating on the scaled Poisson equation (F5) with $\int_{0}^{\rho} d \tilde{\rho} \tilde{\rho}$, we obtain

$$
\rho \frac{\partial \tilde{\psi}}{\partial \rho}=\frac{1+\Delta}{2} \rho^{2}-\int_{0}^{\rho} d \tilde{\rho} \tilde{\rho} e^{-\tilde{\psi}(\tilde{\rho})} .
$$

This equation can be interpreted as the radial force-balance equation for a thermal equilibrium beam [115]. Introducing the scaled radial coordinate $\mathcal{R}$ and density $\mathcal{N}$ defined by

$$
\begin{aligned}
& \mathcal{R}=\frac{1+\Delta}{4} \rho^{2}=\frac{1+\Delta}{4}\left(\frac{r}{\gamma_{b} \lambda_{D}}\right)^{2}, \\
& \mathcal{N}=\frac{e^{-\tilde{\psi}}}{1+\Delta}=\frac{n(r) / \hat{n}}{(1+\Delta)},
\end{aligned}
$$

this radial force-balance equation can be expressed in an equivalent form, with no free parameters, as

$$
\mathcal{R} \frac{\partial}{\partial \mathcal{R}} \mathcal{N}(\mathcal{R})=-\mathcal{R} \mathcal{N}(\mathcal{R})+\mathcal{N}(\mathcal{R}) \int_{0}^{\mathcal{R}} d \tilde{\mathcal{R}} \mathcal{N}(\tilde{\mathcal{R}})
$$

The solution to Eq. (G2) can be expressed as a power series of the form

$$
\mathcal{N}(\mathcal{R})=\sum_{i=0}^{\infty} \alpha_{i} \mathcal{R}^{i}
$$

subject to $\mathcal{N}(\mathcal{R}=0)=(1+\Delta)^{-1}$. Substituting Eq. (G3) 
into Eq. (G2) and equating like powers of $\mathcal{R}$ gives the recursion relations

$$
\begin{aligned}
\alpha_{0} & =(1+\Delta)^{-1} \\
\alpha_{1} & =-\left(\alpha_{0}-\alpha_{0}^{2}\right), \\
\alpha_{2} & =-\frac{1}{2} \alpha_{1}+\frac{3}{4}\left(\frac{1}{2} \alpha_{0} \alpha_{1}+\frac{1}{2} \alpha_{1} \alpha_{0}\right) \\
& =\frac{1}{2}\left(\alpha_{0}-\alpha_{0}^{2}\right)-\frac{3}{4} \alpha_{0}\left(\alpha_{0}-\alpha_{0}^{2}\right), \\
& \vdots \\
\alpha_{i+1} & =-\frac{\alpha_{i}}{i+1}+\frac{i+2}{2(i+1)} \sum_{j=0}^{i} \frac{\alpha_{j} \alpha_{i-j}}{(j+1)(i-j+1)} .
\end{aligned}
$$

Note that all $\alpha_{i}$ with $i \geq 1$ can be calculated in terms of $\alpha_{0}=(1+\Delta)^{-1}$, and thus the coefficients $\alpha_{i}$ can be regarded as known functions of $\Delta$. From Eqs. (G1) and (G3) the thermal equilibrium density profile $n(r)$ can be expressed as

$$
n(r)=\hat{n}+\hat{n}(1+\Delta) \sum_{i=1}^{\infty} \alpha_{i}\left[\frac{1+\Delta}{4}\left(\frac{r}{\gamma_{b} \lambda_{D}}\right)^{2}\right]^{i},
$$

and the solution for $\tilde{\psi}(\rho)=-\log [n(\rho) / \hat{n}]$ is given by

$$
\tilde{\psi}(r)=-\log \left\{1+(1+\Delta) \sum_{i=1}^{\infty} \alpha_{i}\left[\frac{1+\Delta}{4}\left(\frac{r}{\gamma_{b} \lambda_{D}}\right)^{2}\right]^{i}\right\} .
$$

Note from Eq. (G5) that $[\partial n / \partial r]_{r=0}=0$ and $\left[\partial^{2} n / \partial r^{2}\right]_{r=0}=-\Delta \hat{n} /\left(2 \gamma_{b}^{2} \lambda_{D}^{2}\right)$, corresponding to weak downward concavity in the density profile for $\Delta \ll 1$.

The power-series solutions for $n(r)$ and $\tilde{\psi}(r)$ given by Eqs. (G5) and (G6) rapidly converge for large values of $\Delta$ (weak space-charge, see Appendix F) for all $\rho=$ $r /\left(\gamma_{b} \lambda_{D}\right) \in[0, \infty)$. Conversely, for small $\Delta \ll 1$ (strong space-charge, see Appendix F), numerical studies show that the solutions rapidly converge for $\rho$ ranging from the beam center $(\rho=0=r)$ to near the outer radial edge of the beam where the density profile begins falling rapidly. The convergence failure is rapid. For $\Delta \lesssim 0.1$, an accurate approximate formula for the value of $\rho$ where $N=1 / 2$ (denoted by $\rho=\rho_{1 / 2}$ ) is derived in Ref. [116] as

$$
\rho_{1 / 2} \simeq \log \left[\frac{C}{\Delta} \sqrt{2 \pi \log \left(\frac{C}{\Delta}\right)}\right],
$$

where $C=2 \sqrt{3+6 \sqrt{2}}-6 \simeq 0.77799$. The power-series solutions are expected to fail for small $\Delta$ when $\rho$ is near $\rho=\rho_{1 / 2}$.

To circumvent the small $\Delta$ difficulty with direct numerical integration of the thermal equilibrium Poisson equation (F5) and the lack of convergence of the power-series solutions given by Eqs. (G5) and (G6) near the radial edge of the beam (i.e., $\rho \sim \rho_{1 / 2}$ ), we construct numerical solutions as follows. The power-series solutions are employed out to some sufficiently large value of the radial coordinates $\rho=\rho_{c}$, where the series solution is still reliable and highly accurate, but the local variation of the density profile $N$ in $\rho$ is large enough to allow reliable initialization of numerical integration for $\rho \geq \rho_{c}$ with standard methods. Applying the series in small radial steps in $\rho$ out to $\rho=\rho_{c}<\rho_{1 / 2}$ where $N\left(\rho_{c}\right) \simeq 0.98$ appears to be an adequate, simple to implement, criterion. Note from Fig. 17 that the radial edge of the beam is always $3-5$ units in $\rho$ for $\Delta \ll 1$, regardless of the specific value of $\Delta$, so this choice of cutoff is always safely removed from $\rho=$ $\rho_{1 / 2}$.

Rather than numerically integrating the scaled Poisson equation (F5) from $\rho=\rho_{c}$, it is convenient to recast the equation in terms of $N=\exp (-\tilde{\psi})$ instead of $\tilde{\psi}$, and integrate

$$
\frac{\partial^{2} N}{\partial \rho^{2}}+\frac{1}{N}\left(\frac{\partial N}{\partial \rho}\right)^{2}-\frac{1}{\rho} \frac{\partial N}{\partial \rho}=N^{2}-(1+\Delta) N
$$

from the "initial" conditions $N\left(\rho_{c}\right)$ and $\left.\frac{\partial N}{\partial \rho}\right|_{\rho=\rho_{c}}$ calculated from Eq. (G5). In integrating this equation, $(\partial N / \partial \rho)^{2}$ vanishes much faster than $1 / N$ diverges in the low-density tail, so $1 / N$ can be replaced by $1 /(N+\epsilon)$ with $\epsilon$ sufficiently small to avoid challenges with the numerical evaluation of the equations.

The procedure given above can be applied to calculate highly accurate numerical solutions for $N$ or $\psi=-\log N$ for arbitrary values of $\Delta$-however large or small. This method was employed in Ref. [116] to verify approximate solutions constructed for $N$ [see Eq. (F10)].

[1] A. Freidman, D. P. Grote, and I. Haber, Phys. Fluids B 4, 2203 (1992).

[2] D. P. Grote, A. Friedman, I. Haber, W. Fawley, and J.-L. Vay, Nucl. Instrum. Methods Phys. Res., Sect. A 415, 428 (1998).

[3] S. M. Lund, J. J. Barnard, G. D. Craig, A. Friedman, D. P. Grote, H. S. Hopkins, C. Sangster, W. M. Sharp, S. Eylon, T. J. Fessenden, E. Henestroza, S. Yu, and I. Haber, Nucl. Instrum. Methods Phys. Res., Sect. A 415, 345 (1998).

[4] A. V. Fedotov, R. L. Gluckstern, S. S. Kurennoy, and R. D. Ryne, Phys. Rev. ST Accel. Beams 2, 014201 (1999).

[5] D. P. Grote, A. Friedman, G. D. Craig, I. Haber, and W. M. Sharp, Nucl. Instrum. Methods Phys. Res., Sect. A 464, 563 (2001).

[6] J. Qiang, R. Ryne, B. Blind, J. Billen, T. Bhatia, R. Garnett, G. Neuschaefer, and H. Takeda, Nucl. Instrum. Methods Phys. Res., Sect. A 457, 1 (2001).

[7] J. Qiang, P. L. Colestock, D. Gilpatrick, H. V. Smith, T. P. Wangler, and M. E. Schulze, Phys. Rev. ST Accel. Beams 5, 124201 (2002).

[8] R. C. Davidson, I. D. Kaganovich, W. W. Lee, H. Qin, E. A. Startsev, S. Tzenov, A. Friedman, J. J. Barnard, 
R. H. Cohen, D. P. Grote, S. M. Lund, W. M. Sharp, C. M Celata, M. De Hoon, E. Henestroza, E. P. Lee, S. S. Yu, J.-L. Vay, D. R. Welch, D. V. Rose, and C. L. Olson, Laser Part. Beams 20, 377 (2002).

[9] R. A. Kishek, S. Bernal, C. L. Bohn, D. Grote, I. Haber, H. Li, P. G. O'Shea, M. Reiser, and M. Walter, Phys. Plasmas 10, 2016 (2003).

[10] A. Friedman, Nucl. Instrum. Methods Phys. Res., Sect. A 544, 160 (2005).

[11] A. U. Luccio, N. D. Imperio, and N. Malitsky, Nucl. Instrum. Methods Phys. Res., Sect. A 561, 216 (2006).

[12] C. K. Birdsall and A. B. Langdon, Plasma Physics via Computer Simulation (McGraw-Hill, New York, 1985).

[13] R. W. Hockney and J. W. Eastwood, Computer Simulation Using Particles (Taylor \& Francis, New York, 1988).

[14] C. M. Celata, D. P. Grote, and I. Haber, Laser Part. Beams 20, 577 (2002).

[15] S. M. Lund, D. P. Grote, and R. C. Davidson, Nucl. Instrum. Methods Phys. Res., Sect. A 544, 472 (2005).

[16] S. M. Lund and S. R. Chawla, Nucl. Instrum. Methods Phys. Res., Sect. A 561, 203 (2006).

[17] D. P. Grote, E. Henestroza, and J. W. Kwan, Phys. Rev. ST Accel. Beams 6, 014202 (2003).

[18] S. Bernal, P. Chin, R. Kishek, Y. Li, M. Reiser, H. G. Wang, T. Godlove, and I. Haber, Phys. Rev. ST Accel. Beams 1, 044202 (1998).

[19] L. R. Prost, P. A. Seidl, F. M. Bieniosek, C. M. Celata, A. Faltens, D. Baca, E. Henestroza, J. W. Kwan, M. Leitner, W. L. Waldron, R. Cohen, A. Friedman, D. Grote, S. M. Lund, A. W. Molvik, and E. Morse, Phys. Rev. ST Accel. Beams 8, 020101 (2005).

[20] C.Z. Cheng and G. Knorr, J. Comput. Phys. 22, 330 (1976).

[21] E. Sonnendrucker, J. Roche, P. Bertrand, and G. Ghizzo, J. Comput. Phys. 149, 201 (1999).

[22] E. Sonnendrucker, J. J. Barnard, A. Friedman, D. P. Grote, and S. M. Lund, Nucl. Instrum. Methods Phys. Res., Sect. A 464, 470 (2001).

[23] F. Filbet and E. Sonnendrucker, Comput. Phys. Commun. 150, 247 (2003).

[24] F. M. Bieniosek, C. M. Celata, E. Henestroza, J. W. Kwan, L. Prost, P. A. Seidl, A. Friedman, D. P. Grote, S. M. Lund, and I. Haber, Phys. Rev. ST Accel. Beams 8, 010101 (2005).

[25] J.-L. Vay, A. Friedman, and D. P. Grote, in Proceedings of the Seventh International Conference on Computational Accelerator Physics, East Lansing, MI, 2002, edited by M. Berz and K. Makino, Conf. Series No. 175 (Institute of Physics, Bristol, UK, 2005), p. 333.

[26] J.-L. Vay, P. Colella, J. W. Kwan, P. McCorquodale, D. B. Serafini, A. Friedman, D. P. Grote, G. Westenskow, J.-C. Adam, A. Heron, and I. Haber, Phys. Plasmas 11, 2928 (2004).

[27] I. Haber, S. Bernal, C. M. Celta, A. Friedman, D. P. Grote, R. A. Kishek, B. Qinn, P. G. O'Shea, M. Reiser, and J.-L. Vay, Nucl. Instrum. Methods Phys. Res., Sect. A 519, 396 (2004).

[28] S. Hancock, M. Lindroos, and S. Koscielniak, Phys. Rev. ST Accel. Beams 3, 124202 (2000).

[29] A. Friedman, D. P. Grote, C. M. Celata, and J. W. Staples, Laser Part. Beams 21, 17 (2003).
[30] R. C. Davidson, Physics of Nonneutral Plasmas (AddisonWesley, Reading, MA, 1990); (rereleased World Scientific, Singapore, 2001).

[31] M. Reiser, Theory and Design of Charged Particle Beams (John Wiley \& Sons, Inc., New York, 1994).

[32] R. C. Davidson and H. Qin, Physics of Intense Charged Particle Beams in High Energy Accelerators (World Scientific, New York, 2001), and references therein.

[33] M. Tiefenback and D. Keffe, IEEE Trans. Nucl. Sci. 32, 2483 (1985).

[34] M. G. Tiefenback, Ph.D. thesis, University of California at Berkeley, 1986.

[35] S. M. Lund, J. J. Barnard, B. Bukh, S. R. Chawla, and S. H. Chilton, Nucl. Instrum. Methods Phys. Res., Sect. A 577, 173 (2007).

[36] I. Kapchinskij and V. Vladimirskij, in Proceedings of the International Conference on High Energy Accelerators and Instrumentation (CERN Scientific Information Service, Geneva, 1959), p. 274.

[37] R. L. Gluckstern, in Proceedings of the 1970 Proton Linear Accelerator Conference, edited by M. R. Tracy (National Accelerator Laboratory, Batavia, IL, 1971), p. 811.

[38] I. Hofmann, L. J. Laslett, L. Smith, and I. Haber, Part. Accel. 13, 145 (1983).

[39] J. Struckmeier, J. Klabunde, and M. Reiser, Part. Accel. 15, 47 (1984).

[40] R. L. Gluckstern, W.-H. Cheng, and H. Ye, Phys. Rev. Lett. 75, 2835 (1995).

[41] I. Hofmann, Phys. Rev. E 57, 4713 (1998).

[42] S. M. Lund and R. C. Davidson, Phys. Plasmas 5, 3028 (1998).

[43] I. Hofmann, G. Franchetti, O. Boine-Frankenheim, J. Qiang, and R. D. Ryne, Phys. Rev. ST Accel. Beams 6, 024202 (2003).

[44] T.-S. Wang, Phys. Rev. ST Accel. Beams 7, 024201 (2004).

[45] F. J. Sacherer, Ph.D. thesis, University of California, 1968.

[46] V. Danilov, S. Cousineau, S. Henderson, and J. Holmes, Phys. Rev. ST Accel. Beams 6, 094202 (2003).

[47] I. Haber carried out early Vlasov PIC simulations of intense beams with the SHIFTXY code using initial semiGaussian distributions in the 1970s.

[48] S. Bernal, R. A. Kishek, M. Reiser, and I. Haber, Phys. Rev. Lett. 82, 4002 (1999).

[49] R. A. Kishek, S. Bernal, P. G. O'Shea, M. Reiser, and I. Haber, Nucl. Instrum. Methods Phys. Res., Sect. A 464, 484 (2001).

[50] Y.K. Batygin, in Proceedings of the Computational Accelerator Physics Conference, Los Alamos, 1993, edited by R.D. Ryne AIP Conf. Proc. No. 297 (American Institute of Physics, New York, 1994), p. 419.

[51] Y. K. Batygin, Nucl. Instrum. Methods Phys. Res., Sect. A 539, 455 (2005).

[52] P. Channel, Phys. Plasmas 6, 982 (1999).

[53] R. C. Davidson, H. Qin, and P. J. Channell, Phys. Rev. ST Accel. Beams 2, 074401 (1999).

[54] R. C. Davidson, H. Qin, and P. J. Channell, Phys. Rev. ST Accel. Beams 3, 029901 (2000).

[55] S. Tzenov and R. Davidson, Phys. Rev. ST Accel. Beams 
5, 021001 (2002).

[56] K. G. Sonnad and J. R. Cary, in Proceedings of the 2003 Particle Accelerator Conference, Portland, OR (IEEE, Piscataway, NJ, 2003), p. 1536, TPAG047.

[57] K. G. Sonnad and J. R. Cary (unpublished).

[58] E. A. Startsev, R.C. Davidson, and M. Dorf, in Proceedings of the 2009 Particle Accelerator Conference, Vancouver, Canada (IEEE, Piscataway, NJ, 2009), p. FR5PFP042.

[59] J. Struckmeier and I. Hofmann, Part. Accel. 39, 219 (1992).

[60] E. A. Startsev (private communication).

[61] I. Haber, IEEE Trans. Nucl. Sci. 26, 3090 (1979).

[62] I. Haber and A. W. Maschke, Phys. Rev. Lett. 42, 1479 (1979).

[63] Y. K. Batygin, Phys. Rev. E 54, 5673 (1996).

[64] R.D. Ryne tested Langevin procedures in unpublished work.

[65] M. A. Dorf, R. C. Davidson, H. Qin, and E. A. Startsev, in Proceedings of the 2009 Particle Accelerator Conference, Vancouver, Canada (IEEE, Piscataway, NJ, 2009), p. TH6PFP090.

[66] M. A. Dorf, R.C. Davidson, E.A. Startsev, and H. Qin (unpublished).

[67] J. J. Barnard and S. M. Lund, U.S. Particle Accelerator School course notes archived in the laboratory reports: Lawrence Livermore National Laboratory Report No. LLNL-AR-407617 and Lawrence Berkeley National Laboratory Report No. LBNL-1097E, 2008; Lawrence Livermore National Laboratory Report No. UCRL-TM231628 and Lawrence Berkeley National Laboratory Report No. LBNL-62783, 2006; Lawrence Livermore National Laboratory Report No. UCRL-TM-203655 and Lawrence Berkeley National Laboratory, Report No. LBNL-54926, 2004; Lawrence Berkeley National Laboratory Report No. LBNL-49286.2001.

[68] J. J. Barnard and S. M. Lund, Interaction of Intense Charged Particle Beams (University of California at Berkeley, Berkeley, CA, Spring Semester 2009), Nuclear Engineering $290 \mathrm{H}$.

[69] S. M. Lund, French National Institute for Research in Computer Science and Controls (INRIA) School: Modeling and Computational Methods for Kinetic Equations, Rocquencourt, France, 2005.

[70] D. P. Grote, Ph.D. thesis, University of California at Davis, 1994.

[71] D. P. Grote, A. Friedman, I. Haber, and S. Yu, Fusion Eng. Des. 32-33, 193 (1996).

[72] Q. Qian, R.C. Davidson, and C. Chen, Phys. Rev. E 51, R5216 (1995).

[73] S. Machida and M. Ikegami, in Proceedings of the Workshop on Space Charge Physics in High Intensity Hadron Rings (American Institute of Physics, New York, 1998), p. 73.

[74] T. Kikuchi, M. Nakajima, K. Horioka, and T. Katayama, Phys. Rev. ST Accel. Beams 7, 034201 (2004).

[75] T. Kikuchi, T. Someya, S. Kawata, M. Nakajima, K. Horioka, and T. Katayama, Nucl. Instrum. Methods Phys. Res., Sect. A 544, 262 (2005).

[76] T. Kikuchi, T. Someya, S. Kawata, M. Nakajima, K. Horioka, and T. Katayama, Nucl. Instrum. Methods
Phys. Res., Sect. A 558, 122 (2006).

[77] S. M. Lund, O. Boine-Frankenheim, G. Franchetti, I. Hofmann, and P. Spiller, in Proceedings of the 1999 Particle Accelerator Conference (IEEE, Piscataway, NJ, 1999), p. 1785, TUP127.

[78] K. Sakurai, T. Aoki, W.-H. Lee, and K. Kato, Comput. Math. Appl. 43, 621 (2002).

[79] S. M. Lund and B. Bukh, Phys. Rev. ST Accel. Beams 7, 024801 (2004).

[80] S. M. Lund, S. H. Chilton, and E. P. Lee, Phys. Rev. ST Accel. Beams 9, 064201 (2006).

[81] F. J. Sacherer, IEEE Trans. Nucl. Sci. 18, 1105 (1971).

[82] P. M. Lapostolle, IEEE Trans. Nucl. Sci. 18, 1101 (1971).

[83] T. Wangler, K. Crandall, R. Mills, and M. Reiser, IEEE Trans. Nucl. Sci. 32, 2196 (1985).

[84] J. Struckmeier and M. Reiser, Part. Accel. 14, 227 (1984).

[85] I. Haber applied the method described in the Vlasov PIC code SHIFTXY in the 1970s.

[86] F. J. Sacherer, Technical Report No. CERN-SI-INT-DL70-5, 1970.

[87] T. K. Fowler, J. Math. Phys. 4, 559 (1963).

[88] C. S. Gardner, Phys. Fluids 6, 839 (1963).

[89] R. C. Davidson, Phys. Rev. Lett. 81, 991 (1998).

[90] S. M. Lund, Phys. Rev. ST Accel. Beams 10, 064203 (2007).

[91] S. M. Lund, J. J. Barnard, and J. Miller, in Proceedings of the 1995 Particle Accelerator Conference, Dallas, TX (IEEE, Piscataway, NJ, 1995), p. 3278.

[92] B. L. Bogema and R. C. Davidson, Phys. Fluids 13, 2772 (1970).

[93] R. B. Miller, An Introduction to the Physics of Intense Charged Particle Beams (Plenum Press, New York, 1982).

[94] R. A. Kishek, P. G. O'Shea, and M. Reiser, Phys. Rev. Lett. 85, 4514 (2000).

[95] W.H. Press, B.P. Flannery, S. A. Teukolsky, and W. A. Vetterling, Numerical Recipes in FORTRAN 77: The Art of Scientific Computing (Cambridge University Press, New York, 1992), 2nd ed..

[96] Y. K. Batygin (private communication).

[97] H. Wiedemann, Particle Accelerator Physics: Basic Principles and Linear Beam Dynamics (Springer-Verlag, New York, 1993).

[98] D. A. Edwards and L. C. Teng, IEEE Trans. Nucl. Sci. 20, 885 (1973).

[99] D. Neuffer, IEEE Trans. Nucl. Sci. 26, 3031 (1979).

[100] J. B. Marion, Classical Dynamics of Particles and Systems (Academic Press, New York, 1970), 2nd ed.

[101] E. P. Lee (private communication).

[102] H. Wiedemann, Particle Accelerator Physics II: Nonlinear and Higher-Order Beam Dynamics (Springer-Verlag, New York, 1995).

[103] H. Goldstein, Classical Mechanics (Addison Wesley, Reading, MA, 1980), 2nd ed.

[104] I. Hofmann, in Advances in Electronics and Electron Physics, Supplement 13C, edited by A. Septier (Academic Press, New York, 1983), pp. 49-140.

[105] R. C. Davidson, H. Qin, S. I. Tzenov, and E. A. Startsev, Phys. Rev. ST Accel. Beams 5, 084402 (2002).

[106] E. A. Startsev and R. C. Davidson, Phys. Rev. ST Accel. Beams 6, 044401 (2003). 
[107] H. Okamoto, Nucl. Instrum. Methods Phys. Res., Sect. A 332, 1 (1993).

[108] Handbook of Mathematical Functions with Formulas, Graphs, and Mathematical Tables, edited by M. Abramowitz and I. A. Stegun (Dover, New York, 1972), 9th ed.

[109] R. C. Davidson, J. Plasma Phys. 6, 229 (1971).

[110] D. H. E. Dubin and T. M. O’Neil, Rev. Mod. Phys. 71, 87 (1999).

[111] M. Reiser and N. Brown, Phys. Rev. Lett. 71, 2911 (1993).
[112] J.D. Lawson, The Physics of Charged-Particle Beams (Clarendon Press, Oxford, 1977).

[113] R. C. Davidson and H. Qin, Phys. Rev. ST Accel. Beams 2, 114401 (1999).

[114] R. C. Davidson, H. Qin, and S. Lund, Phys. Rev. ST Accel. Beams 6, 024402 (2003).

[115] R. C. Davidson and S. M. Lund, in Advances in Plasma Physics, edited by N. Fisch (American Institute of Physics, New York, 1994), Vol. 314, p. 1.

[116] E. A. Startsev and S. M. Lund, Phys. Plasmas 15, 043101 (2008). 